\title{
TILTED ALGEBRAS
}

BY

\author{
DIETER HAPPEL AND CLAUS MICHAEL RINGEL
}

\begin{abstract}
Let $A$ be a finite dimensional hereditary algebra over a field, with $n$ simple $A$-modules. An $A$-module $T_{A}$ with $n$ pairwise nonisomorphic indecomposable direct summands and satisfying $\operatorname{Ext}^{1}\left(T_{A}, T_{A}\right)=0$ is called a tilting module, and its endomorphism ring $B$ is a tilted algebra. A tilting module defines a (usually nonhereditary) torsion theory, and the indecomposable $B$-modules are in one-to-one correspondence to the indecomposable $A$-modules which are either torsion or torsionfree. One of the main results of the paper asserts that an algebra of finite representation type with an indecomposable sincere representation is a tilted algebra provided its Auslander-Reiten quiver has no oriented cycles. In fact, tilting modules are introduced and studied for any finite dimensional algebra, generalizing recent results of Brenner and Butler.
\end{abstract}

Let $A$ be a finite dimensional algebra (associative, with 1). All $A$-modules will be finite dimensional, and homomorphisms will be written on the opposite side of the scalars. Thus, given an $A$-module $M_{A}$, with endomorphism ring $B=\operatorname{End}\left(M_{A}\right)$, we also can consider the $B$-module ${ }_{B} M$. If in this situation, the canonical map $A \rightarrow \operatorname{End}\left({ }_{B} M\right)$ is an isomorphism, we just will identify $A$ with $\operatorname{End}\left({ }_{B} M\right)$.

A module $T_{A}$ will be called a tilting module provided it satisfies the following three properties:

(1) There is an exact sequence $0 \rightarrow P_{A}^{\prime \prime} \rightarrow P_{A}^{\prime} \rightarrow T_{A} \rightarrow 0$, with $P^{\prime}, P^{\prime \prime}$ projective (thus, p.d. $T_{A} \leqslant 1$ ).

(2) $\operatorname{Ext}^{1}\left(T_{A}, T_{A}\right)=0$.

(3) There is an exact sequence $0 \rightarrow A_{A} \rightarrow T_{A}^{\prime} \rightarrow T_{A}^{\prime \prime} \rightarrow 0$, with $T^{\prime}, T^{\prime \prime}$ being direct sums of summands of $T$.

Given a tilting module $T_{A}$, one may consider two full subcategories of the category $\Re_{A}$ of all right $A$-modules, namely the category $\mathscr{T}\left(T_{A}\right)$ of all modules $M_{A}$ generated by $T_{A}$ and the category $\mathscr{F}\left(T_{A}\right)$ of all modules $M_{A}$ satisfying $\operatorname{Hom}\left(T_{A}, M_{A}\right)=0$. It is easy to see that $\mathscr{T}\left(T_{A}\right)$ is the class of torsion modules, and $\mathscr{F}\left(T_{A}\right)$ the class of torsionfree modules of a torsion theory $\left(\mathscr{T}\left(T_{A}\right), \mathscr{F}\left(T_{A}\right)\right)$. The main result on tilting modules is essentially due to Brenner and Butler [6].

TheOrem of BrenNer-Butler. Let $T_{A}$ be a tilting module, and $B=\operatorname{End}\left(T_{A}\right)$. Then ${ }_{B} T$ also is a tilting module, and $A=\operatorname{End}\left({ }_{B} T\right)$, canonically. The categories $\mathcal{T}\left(T_{A}\right)$ and $\widetilde{\mathcal{T}}\left({ }_{B} T\right)$ are dual to each other; similarly, the categories $\mathcal{F}\left(T_{A}\right)$ and $\mathscr{F}\left({ }_{B} T\right)$ are dual to each other.

Received by the editors November 19, 1980 and, in revised form, September 9, 1981.

1980 Mathematics Subject Classification. Primary 16A46, 16A63, 16A64; Secondary 16A60, 16A65, 16A89, 18E40. 
In [6], Brenner and Butler used a more restrictive notion of tilting modules; however, their arguments remain valid in the general situation. Also, they only considered the duality between the categories of torsion modules, and used a rather different way of formulation. For the benefit of the reader, we will give the full proof of the theorem.

We also will consider a linear transformation $f$ which relates the dimension type of corresponding modules in $\mathcal{T}\left(T_{A}\right)$ and $\mathcal{T}\left({ }_{B} T\right)$ or in $\mathscr{F}\left(T_{A}\right)$ and $\mathscr{F}\left({ }_{B} T\right)$. In case the Euler characteristic is defined, $f$ actually will be an isometry (see $\S 3$ ). In particular, we see that in case there exists a tilting module $T_{A}$ with $B=\operatorname{End}\left(T_{A}\right)$, the number of simple $A$-modules coincides with the number of simple $B$-modules.

The main parts of the present paper will deal with the special case of $A$ being hereditary. If the finite dimensional algebra $B$ is of the form $B=\operatorname{End}\left(T_{A}\right)$, where $T_{A}$ is a tilting module and $A$ is hereditary, then $B$ will be called a tilted algebra. The starting point of our investigation is the following observation.

TheOrem. Let $A$ be hereditary, and $T_{A}$ a tilting module, with $B=\operatorname{End}\left(T_{A}\right)$. Then $\left(\mathscr{T}\left({ }_{B} T\right), \mathscr{F}\left({ }_{B} T\right)\right)$ is a splitting torsion theory.

Recall that a torsion theory is said to be splitting in case any indecomposable module is either a torsion module or torsionfree. Note that the theorem furnishes a one-to-one correspondence between the indecomposable $B$-modules and certain indecomposable $A$-modules. In particular, if $A$ is of finite representation type, then also $B$ is of finite representation type.

We will derive some important properties of a tilted algebra $B$. We will show that the quiver of $B$ has no oriented cycles (Corollary 4.2), and that gl. $\operatorname{dim} B \leqslant 2$ (Theorem 5.2).

Conversely, we will give sufficient conditions for $B$ in order to be a tilted algebra. A right module $M_{A}$ will be called sincere provided any simple $A$-module appears as a composition factor of $M_{A}$. We will introduce the notion of a complete slice (see Definition 7.1) and show that in case one component of the Auslander-Reiten quiver of $B$ contains as well a complete slice as well as all indecomposable projective modules, then $B$ is a tilted algebra (Theorem 7.2). As a consequence, we will see that an algebra of finite representation type with an indecomposable sincere representation is a tilted algebra if and only if its Auslander-Reiten quiver has no oriented cycle. In fact, in this situation, one has the following result:

THEOREM. Let $B$ be of finite representation type, and assume its Auslander-Reiten quiver has no oriented cycle. Also assume that ${ }_{B} N$ is an indecomposable sincere representation. Then there exists a hereditary algebra $A$, a tilting module $T_{A}$, and a primitive idempotent $e$ of $A$ such that $B=\operatorname{End}\left(T_{A}\right)$ and ${ }_{B} N={ }_{B} T e$.

As a consequence, we conclude the following: if $B$ is of finite representation type, and its Auslander-Reiten quiver has no oriented cycle, then the indecomposable $B$-modules are uniquely determined by their composition factors.

Tilted algebras seem to be of interest for several reasons: First of all, they provide a rather general setting for dealing with certain special types of algebras, for example 
trees with relations of finite representation type, and the results of this paper can be used in order to give a complete classification of all their indecomposable representations [17]. Also, one may use the theory of tilted algebras in order to construct algebras with prescribed structure of some of the components of their AuslanderReiten quivers. Finally, and most important, representation theory of tilted algebras should provide a better insight into the structure of representations of arbitrary algebras of finite representation type using recently developed covering techniques. For a discussion of the history of the use of special tilting modules, we refer to [6]. An account for constructing tilting modules will be given in [18].

\section{Preliminaries.}

(1.1) Notation. Most modules considered will be right modules, and $\mathfrak{M}_{A}$ will denote the category of all right $A$-modules. In case we have to deal with left modules, we will stress this fact explicitly. Of course, there always will be a bimodule involved in our investigation, which usually will be denoted by ${ }_{B} T_{A}$ or ${ }_{A} U_{B}$.

If $M$ is a module, we denote by $\operatorname{End}(M)$ its endomorphism $\operatorname{ring}$, by $\operatorname{rad} M$ its radical, by soc $M$ its socle, by $|M|$ its length, and by p.d. $M$ its projective dimension. Also, let add $M$ be the class of all modules which are direct sums of direct summands of $M$. We denote by $X \approx Y$ that $X, Y$ are isomorphic (as groups, or modules whatever we deal with). We usually will not distinguish between a module $M$ and the isomorphism class of all modules isomorphic to $M$. If $f: M \rightarrow M^{\prime}$ is a module homomorphism (we just will say a “map"), then $\operatorname{Ker} f$ denotes its kernel, Cok $f$ its cokernel. A module $X$ is said to be generated by the module $M$, provided $X$ is an epimorphic image of some direct sum $\oplus M$, and $X$ is said to be cogenerated by $M$, provided $X$ is a submodule of some direct sum $\oplus M$.

There always will be two algebras $A$ and $B$ involved in our consideration. In $\Re_{A}$, we usually will denote projective modules by $P, P^{\prime}, P_{i}, \ldots$, injective modules by $I, I^{\prime}, I_{i}, \ldots$, and elements of add $T_{A}$ by $T^{\prime}, T^{\prime \prime}, T_{i}, \ldots$ The simple $A$-modules will be denoted by $R$ or $R(a)$, where $a$ runs through a fixed index set, $P(a)$ will be the projective module with $P(a) / \operatorname{rad} P(a)=R(a)$, and $I(a)$ will be the injective module with soc $I(a)=R(a)$. Similarly, in $\Re_{B}$, projective modules will be denoted by $Q, Q^{\prime}, Q_{i}, \ldots$, injective modules by $J, J^{\prime}, J_{i}, \ldots$, and elements of add $U_{B}$ by $U^{\prime}, U^{\prime \prime}, U_{i}, \ldots$ The simple $B$-modules will be denoted by $S$ or $S(i)$, where $i$ runs through a fixed index set (usually $\{1, \ldots, n\}), Q(i)$ will be the projective module with $Q(i) / \operatorname{rad} Q(i)=S(i)$, and $J(i)$ will be the injective module with $\operatorname{soc} J(i)=S(i)$.

We denote by $G_{0}(A)$ the Grothendieck group of $A$, it can be identified with the free abelian group having the set $\{R(a) \mid a\}$ of all (isomorphism classes of) simple $A$-modules as basis. Using this basis, $G_{0}(A)$ becomes a partially ordered group. If $M$ is an $A$-module, we denote by $\operatorname{dim} M$ the corresponding element of $G_{0}(A)$, it will be called the dimension vector of $M$. The coefficients of $\operatorname{dim} M$ with respect to the canonical basis can be calculated as follows: $(\operatorname{dim} M)_{a}$ is the number of composition factors of the form $R(a)$ in any fixed composition series, and we have

$$
(\operatorname{dim} M)_{a}=\left|\operatorname{Hom}_{A}(P(a), M)_{\text {End } P(a)}\right|=\mid \text { End } I(a) \operatorname{Hom}_{A}(M, I(a)) \mid .
$$

Note that $M_{A}$ is sincere iff $(\operatorname{dim} M)_{a} \neq 0$ for all $a$. 
Finally, note that $D$ denotes the duality with respect to the basefield $k$. Thus $D(M)=\operatorname{Hom}_{k}(M, k)$. [More generally, we may assume that we deal with an artin algebra $A$, then $D(M)=\operatorname{Hom}_{Z}(M, I(\bar{Z}))$, where $Z$ is the center of $A, \bar{Z}=Z / \operatorname{rad} Z$, and $I(\bar{Z})$ is the injective envelope of $\bar{Z}$ as a $Z$ - module.]

(1.2) Auslander-Reiten quiver. Let us recall the main notions and results from [1].

Let $A$ be a finite dimensional algebra. A map $f: M \rightarrow M^{\prime}$ is called irreducible provided $f$ is neither split mono, nor split epi, and for every factorization $f=f^{\prime \prime} f^{\prime}, f^{\prime}$ is split mono or $f^{\prime \prime}$ is split epi. If $M, M^{\prime}$ are indecomposable, then there exists an irreducible map $M \rightarrow M^{\prime}$ if and only if the bimodule $\operatorname{Irr}\left(M, M^{\prime}\right)$ of irreducible maps is nonzero. [Recall that $\operatorname{Irr}\left(M, M^{\prime}\right)=\Re\left(M, M^{\prime}\right) / \mathcal{R}^{2}\left(M, M^{\prime}\right)$, for $M, M^{\prime}$ indecomposable, where $R\left(M, M^{\prime}\right)$ is the set of noninvertible maps, and $\Re^{2}\left(M, M^{\prime}\right)$ the set of maps of the form $\sum f_{i} g_{i}$, with $f_{i} \in \Re\left(M, X_{i}\right), g_{i} \in \Re\left(X_{i}, M\right)$ and all $X_{i}$ indecomposable. Clearly, this is an $\operatorname{End}\left(M^{\prime}\right)-\operatorname{End}(M)$-bimodule which actually is annihilated by the radicals of $\operatorname{End}\left(M^{\prime}\right)$ and $\operatorname{End}(M)$.]

If $M$ is indecomposable, a map $g: M^{\prime} \rightarrow M$ is called right almost split, provided $g$ is not split epi and for every map $h: X \rightarrow M$ which is not split epi, there exists $h^{\prime}$ with $h=g h^{\prime}$. If in addition $M^{\prime}$ is of smallest possible length, then $g$ is called minimal right almost split. Note that for every $M$, there always exists a minimal right almost split map $M^{\prime} \rightarrow M$, and it is unique up to isomorphism. Also, a map $h: X \rightarrow M$ with $X \neq 0$ is irreducible if and only if there exists $h^{\prime}: X^{\prime} \rightarrow M$ such that $\left(h h^{\prime}\right)$ : $X \oplus X^{\prime} \rightarrow M$ is minimal right almost split. Actually, if $\oplus X_{i} \rightarrow M$ is minimal right almost split, with all $X_{i}$ indecomposable, then the number of $X_{i}$ isomorphic to a fixed $X$ is precisely $\left|\operatorname{Irr}(X, M)_{\operatorname{End}(X)}\right|$, see [14]. The kernel $\operatorname{Ker} g$ of a minimal right almost split map $g: M^{\prime} \rightarrow M$ is denoted by $\tau M$ and called the Auslander-Reiten translate of $M$. There are two possibilities: either $M=P$ is projective, then $\tau P=0$, and the minimal right almost split map is just given by the inclusion $\operatorname{rad} P \rightarrow P$, or else $M$ is not projective, then $\tau M$ is indecomposable and we obtain an exact sequence

$$
0 \rightarrow \tau M \rightarrow M^{\prime} \stackrel{g}{\rightarrow} M \rightarrow 0
$$

which is called the Auslander-Reiten sequence ending with $M$. Dually, for every indecomposable module $M$, there also exists a unique minimal left almost split map $M \rightarrow M^{\prime \prime}$, and its cokernel is denoted by $\tau^{-1} M$. If $M=I$ is injective, then the minimal left almost split map starting with $I$ is just the canonical projection $I \rightarrow I / \operatorname{soc} I$. If $M$ is not injective, then $\tau^{-1} M$ is indecomposable and we obtain the exact sequence

$$
0 \rightarrow M \stackrel{f}{\rightarrow} M^{\prime \prime} \rightarrow \tau^{-1} M \rightarrow 0
$$

which is the Auslander-Reiten sequence ending with $\tau^{-1} M$. In particular, $\tau \tau^{-1} M \approx M$ for $M$ indecomposable and not injective, and $\tau^{-1} \tau M \approx M$ for $M$ indecomposable and not projective. Note that the module $\tau M$ can be calculated as follows: choose a projective resolution

$$
P^{\prime} \stackrel{p}{\rightarrow} P \rightarrow M \rightarrow 0
$$


of $M$, with $P^{\prime}$ minimal, and apply $\operatorname{Hom}_{A}\left(-, A_{A}\right)$. The cokernel $\operatorname{Cok} \operatorname{Hom}\left(p, A_{A}\right)$ is called the transpose of $M$ and denoted by $\operatorname{Tr} M$. Then $\tau M=D \operatorname{Tr} M$, and similarly $\tau^{-1} M=\operatorname{Tr} D M$. Finally, we remark that for $X, Y$ indecomposable, $\operatorname{Ext}^{1}(X, Y)$ can be considered as a factor group both of $D \operatorname{Hom}(Y, \tau X)$ and of $D \operatorname{Hom}\left(\tau^{-1} Y, X\right)$ (sometimes, these groups actually are isomorphic, see for example 2.5).

The Auslander-Reiten quiver of $B$ has as vertices the (isomorphism classes of) indecomposable $B$-modules, and there is an arrow $\rightarrow$ going from $M$ to $M^{\prime}$ provided there exists an irreducible map $M \rightarrow M^{\prime}$. The existence of minimal almost split maps, and their properties, show that the Auslander-Reiten quiver is locally finite, thus its connected components contain at most countably many vertices.

In case $A$ is connected (i.e. has no central idempotents different from 0,1 ), and $A$ is of finite representation type, then its Auslander-Reiten quiver is connected. If $A$ is connected and not of finite representation type, then no component of its Auslander-Reiten quiver is finite [15].

Note that the Auslander-Reiten quiver is endowed with the additional, partially defined endo-map $\tau$; it is defined for all vertices which correspond to nonprojective modules, and has the following property: If $X, Y$ are vertices of the AuslanderReiten quiver, and $X$ is not projective, then there is an arrow $Y \rightarrow X$ if and only if there is an arrow $\tau X \rightarrow Y$.

Very often, we will have to consider paths in the Auslander-Reiten quiver of an algebra $A$. Note that in case $X$ is a $\tau$-periodic module, then we obtain an oriented cycle. [Namely, if $\tau X \neq 0$, consider the corresponding Auslander-Reiten sequence $0 \rightarrow \tau X \rightarrow \oplus Y_{i} \rightarrow X \rightarrow 0$, with $Y_{i}$ indecomposable, so we have a path $\tau X \rightarrow Y_{1} \rightarrow X$. Now if $\tau^{p} X \neq 0$ for some $p>0$, we obtain in this way a path of length $2 p$ starting with $\tau^{p} X$ and ending with $X$. Thus, if $\tau^{p} X \approx X$, this is an oriented cycle.] In case $X, Y$ are indecomposable modules and there exists a path from $X$ to $Y$ in the Auslander-Reiten quiver, then $X$ will be called a predecessor of $Y$. Note that in case an indecomposable module $Y$ has only finitely many predecessors, and $\operatorname{Hom}(X, Y)$ $\neq 0$ for some indecomposable module $X$, then there is a path from $X$ to $Y$. [Namely, assume there is no such path. Then we define inductively a path

$$
Y_{m} \stackrel{g_{m}}{\rightarrow} Y_{m-1} \rightarrow \cdots \rightarrow Y_{1} \stackrel{g_{1}}{\rightarrow} Y_{0}=Y
$$

of irreducible maps, and maps $f_{m}: X \rightarrow Y_{m}$ such that $g_{1} \cdots g_{m} f_{m} \neq 0$. We take some $0 \neq f_{0}: X \rightarrow Y=Y_{0}$, and if $g_{1}, \ldots, g_{m}, f_{m}$ are defined, let $\left(h_{t}\right)_{t}: \oplus Z_{t} \rightarrow Y_{m}$ be minimal right almost split, all $Z_{t}$ indecomposable. There exist $h_{t}^{\prime}$ with $f_{m}=\Sigma h_{t} h_{t}^{\prime}$, and $g_{1} \cdots g_{m} h_{t_{0}} h_{t_{0}}^{\prime} \neq 0$ for some $t_{0}$. Let $Y_{m+1}=Z_{t_{0}}, g_{m+1}=h_{t_{0}}, f_{m+1}=h_{t_{0}}^{\prime}$. However, since there are only finitely many predecessors of $Y$, say with length bounded by $b$, any such path has length $<2^{b}$ [10], contrary to our construction.]

(1.3) Preprojective components. A component $\mathcal{C}$ of the Auslander-Reiten quiver of the algebra $A$ will be called preprojective provided there is no oriented cycle in $C$ and any module of $C$ is of the form $\tau^{-t} P$, for some $t \in \mathbf{N}$ and some indecomposable projective module $P$. [It is not difficult to see that a component $\mathcal{C}$ is preprojective if and only if $C$ has no oriented cycles and all modules in $C$ are preprojective in the sense of Auslander and Smalø.]. 
If $C$ is the preprojective component, and $Y$ is a module in $C$, then $Y$ has only finitely many predecessors. [Namely, consider a path

$$
Y_{m} \rightarrow Y_{m-1} \rightarrow \cdots \rightarrow Y_{1} \rightarrow Y_{0}=Y
$$

in $e$. All $Y_{i}$ are of the form $\tau^{-t_{i}} P_{i}$ for some $t_{i} \in \mathbf{N}$, and some indecomposable projective module $P_{i}$. If $i>j$, and $P_{i}=P_{j}$, then $t_{i}<t_{j}$, since otherwise we would obtain the oriented cycle

$$
\tau^{-t_{i}} P_{i}=Y_{i} \rightarrow \cdots Y_{j}=\tau^{-t_{j}} P_{j} \rightarrow \cdots \rightarrow \tau^{-t_{i}} P_{j},
$$

impossible. Since there are only finitely many indecomposable projective modules, we conclude that any path

$$
\cdots \rightarrow Y_{m} \rightarrow Y_{m-1} \rightarrow \cdots \rightarrow Y_{1} \rightarrow Y_{0}=Y
$$

terminates after a finite number of steps, thus $Y$ has only finitely many predecessors, due to König's graph theorem.] As a consequence, we have:

Lemma. Let $Y$ be an indecomposable module belonging to a preprojective component e. Then if $X$ is an indecomposable module, any homomorphism $X \rightarrow Y$ is a sum of compositions of irreducible maps. Thus, if $\operatorname{Hom}(X, Y) \neq 0$, then there is a path from $X$ to $Y$, thus $X \in \mathcal{C}$. In particular, if $Y$ belongs to a preprojective component of the Auslander-Reiten quiver of $B$, and $\bar{B}=B /$ Ann $Y$, then $Y$ also belongs to a preprojective component of the Auslander-Reiten quiver of $\bar{B}$.

Proof. Consider the predecessors of $Y$ which also are $\bar{B}$-modules. This is a finite set closed under predecessors and containing all indecomposable projective $\bar{B}$-modules. Now it is easy to see that the component of the Auslander-Reiten quiver of $\bar{B}$ containing $Y$ consists of modules of the form $\tau_{B}^{=t} P$ with $P$ an indecomposable projective $\bar{B}$-module (see [8]), and this is a preprojective component.

(1.4) Torsion theories. Let $\mathfrak{X}, \mathcal{Y}$ be two full subcategories of $\mathfrak{\pi}_{A}$. Then ( $\left.\mathfrak{X}, \mathscr{Y}\right)$ is called a torsion theory, provided $\operatorname{Hom}(X, Y)=0$ for all modules $X \in \mathfrak{X}$ and $Y \in \mathcal{Y}$, and both $\mathcal{X}$ and $\mathcal{Y}$ are maximal with this property (thus, if $\operatorname{Hom}(X, M)=0$ for all $X \in \mathcal{X}$, then $M \in \mathcal{Y}$, whereas if $\operatorname{Hom}(M, Y)=0$ for all $Y \in \mathcal{Y}$, then $M \in \mathfrak{X})$.

If we deal with a fixed torsion theory ( $\mathcal{X}, \mathcal{Y})$, then the modules in $\mathscr{X}$ are called torsion modules, those of $\mathcal{Y}$ are called torsionfree. Given any module $M$, there exists a unique largest torsion submodule $X$ of $M$, and $M / X$ is torsionfree, and, in fact, the largest torsionfree factor module of $M$. The torsion theory $(\mathfrak{X}, \mathscr{Y})$ is called splitting provided any module is a direct sum of a module from $\mathscr{X}$ and a module from $\mathscr{Y}$.

[Note that in contrast to most investigations concerned with torsion theories the torsion theories we will have to deal with, will not be hereditary.]

If $T_{A}$ is a module with p.d. $T_{A} \leqslant 1$ and $\operatorname{Ext}_{A}^{1}(T, T)=0$, then we can define a torsion theory as follows: Let $\mathcal{T}(T)$ be the full subcategory of all modules generated by $T_{A}$, and $\mathscr{F}(T)$ the full subcategory of all modules $Y_{A}$ with $\operatorname{Hom}\left(T_{A}, Y_{A}\right)=0$. Then $(\mathscr{T}(T), \mathscr{F}(T))$ is a torsion theory. [Namely, let us show that $\mathscr{T}(T)$ is closed under extensions. A class of modules closed under factor modules, and extensions always is the class of torsion modules of some torsion theory, and the corresponding torsionfree modules have to be the modules in $\mathscr{F}(T)$. 
Thus, consider an exact sequence

$$
0 \rightarrow X_{1} \rightarrow X \rightarrow X_{2} \rightarrow 0,
$$

and epimorphisms $f_{i}: T_{i} \rightarrow X_{i}$, where $T_{1}, T_{2}$ are direct sums of copies of $T$. We first form the induced sequence with respect to $f_{2}$, and then we use p.d. $T_{2} \leqslant 1$ in order to obtain the upper exact sequence of the following diagram:

$$
\begin{array}{ccccccccc}
0 & \rightarrow & T_{1} & \rightarrow & X^{\prime \prime} & \rightarrow & T_{2} & \rightarrow & 0 \\
& & \downarrow f_{1} & & \downarrow f_{1}^{\prime} & & \| & & \\
0 & \rightarrow & X_{1} & \rightarrow & X^{\prime} & \rightarrow & T_{2} & \rightarrow & 0 \\
& & \| & & \downarrow f_{2}^{\prime} & & & & \\
& & & & & \\
0 & \rightarrow & X_{2} & \rightarrow & X & \rightarrow & X_{2} & \rightarrow & 0
\end{array}
$$

Since $\operatorname{Ext}^{1}\left(T_{2}, T_{1}\right)=0$, we see that the upper sequence splits; thus $X^{\prime \prime}=T_{1} \oplus T_{2}$ is a direct sum of copies of $T$, and $f_{2}^{\prime} f_{1}^{\prime}$ is an epimorphism.]

We also will need that, in the situation above, the subcategory $\mathscr{T}(T)$ has relative Auslander-Reiten sequences, this follows from a rather general result of Auslander and Smalø [2]. We recall the definitions: Let $\mathscr{Z}$ be an extension closed full subcategory. A map $g: M^{\prime} \rightarrow M$ in $\mathscr{Z}$ is called right almost split, provided $g$ is not split epi, and for any $h: X \rightarrow M$ in $\mathscr{Z}$ which is not split epi, there exists $h^{\prime}$ with $h=g h^{\prime}$. Dually, a map $f: M \rightarrow M^{\prime \prime}$ in $\mathscr{Z}$ is left almost split, provided $f$ is not split mono, and for any $h: M \rightarrow X$ in $\mathscr{Z}$ which is not split mono, there exists $h^{\prime}$ with $h=h^{\prime} f$. A relative Auslander-Reiten sequence in $\mathscr{Z}$ is an exact sequence

$$
0 \rightarrow M_{1} \stackrel{f}{\rightarrow} M_{2} \stackrel{g}{\rightarrow} M_{3} \rightarrow 0
$$

with all $M_{i}$ in $\mathscr{Z}$ such that $f$ is left almost split, $g$ right almost split. Now $\mathscr{Z}$ is said to have relative Auslander-Reiten sequences provided

(1) If $Z$ is indecomposable and in $\mathscr{Z}$, there is a right almost split map $Z^{\prime} \rightarrow Z$ in $\mathscr{Z}$, and also a left almost split map $Z \rightarrow Z^{\prime \prime}$ in $\mathscr{Z}$.

(2) If $M_{1}$ is indecomposable and in $\mathscr{Z}$, and $\operatorname{Ext}^{1}\left(M, M_{1}\right) \neq 0$ for some $M$ in $\mathscr{Z}$, then there exists a relative Auslander-Reiten sequence $(*)$.

(3) If $M_{3}$ is indecomposable and in $\mathscr{Z}$, and $\operatorname{Ext}^{1}\left(M_{3}, M\right) \neq 0$ for some $M$ in $\mathscr{Z}$, then there exists a relative Auslander-Reiten sequence $(*)$.

[In order to see that $\widetilde{T}\left(T_{A}\right)$ has Auslander-Reiten sequences, one uses the equivalence of (a) and (c) in 5.9, assertion (b) of 5.10, and 2.4 of [2]. Note that condition (a) of 5.9 is a direct consequence of p.d. $T_{A} \leqslant 1$ and $\operatorname{Ext}_{A}^{1}(T, T)=0$.]

(1.5) Euler characteristic. We say that the Euler characteristic is defined for $A$ in case the dimension vectors $\operatorname{dim} P(a)$ of the indecomposable projective $A$-modules are linearly independent in $G_{0}(A)$. In this case, let

$$
\langle\operatorname{dim} P(a), \operatorname{dim} P(b)\rangle=\operatorname{dim}_{k} \operatorname{Hom}_{A}(P(a), P(b)),
$$

and extend $\langle$,$\rangle bilinearly to all of G_{0}(A)$. In this way, we obtain a bilinear form

$$
\langle,\rangle: G_{0}(A) \times G_{0}(A) \rightarrow \mathbf{Q}
$$

which will be called the Euler characteristic of $A$. 
Note that if $A$ has finite global dimension, then the Euler characteristic is defined for $A$, and we have

$$
\langle\operatorname{dim} X, \operatorname{dim} Y\rangle=\sum_{i \geqslant 0}(-1)^{i} \operatorname{dim}_{k} \operatorname{Ext}^{i}(X, Y)
$$

for all $A$-modules $X, Y$. [Namely, any simple $A$-module has a finite projective resolution say

$$
0 \rightarrow P_{m} \rightarrow \cdots \rightarrow P_{1} \rightarrow P_{0} \rightarrow S(a) \rightarrow 0
$$

thus $\operatorname{dim} S(a)=\sum(-1)^{i} \operatorname{dim} P_{i}$ lies in the subgroup of $G_{0}(A)$ generated by the various dimension vectors $\operatorname{dim} P(a)$, thus the Euler characteristic is defined for $A$. Now both sides of $(*)$ are additive on exact sequences and coincide on the projective modules, thus we have equality.]

There are also algebras of infinite global dimension for which the Euler characteristic is defined. For example, let $A$ be a local algebra with $P=A_{A}$ of length $n$. Then, $\operatorname{dim} P=n \cdot \operatorname{dim} S$ where $S$ is the simple $A$-module, thus the Euler characteristic is defined. It can be calculated on the generator $\operatorname{dim} S$ of $G_{0}(A)$, as follows:

$$
\begin{aligned}
\langle\operatorname{dim} S, \operatorname{dim} S\rangle & =\frac{1}{n^{2}}\langle\operatorname{dim} P, \operatorname{dim} P\rangle \\
& =\frac{1}{n^{2}} \operatorname{dim} \text { End }(P)=\frac{1}{n} .
\end{aligned}
$$

(1.6) Hereditary algebras. Assume now $A$ is hereditary. Let $P(a), a \in \Gamma_{0}$, be the indecomposable projective modules. We will consider $\Gamma_{0}$ as a set of vertices of a quiver $\Gamma$, with arrows $a \rightarrow b$ provided $P(a)$ is a direct summand of $\operatorname{rad} P(b)$. Note that this quiver never has oriented cycles. A source of this quiver just corresponds to a simple projective module. [In this way we just consider the full subquiver of the Auslander-Reiten quiver of $A$ defined by the indecomposable projective modules, and transfer the quiver structure to the index set $\Gamma_{0}$. Note that in case $A$ is the path algebra of a quiver $\Delta$, then $\Gamma$ is obtained from $\Delta$ by reversing all arrows and replacing multiple arrows by a single one.] Note that $\Gamma$ becomes a valued graph [7] if we define $d_{a b}, d_{a b}^{\prime}$ as follows:

$$
\operatorname{rad} P(b)=\bigoplus_{a} P(a)^{d_{a b}}, \quad I(a) / \operatorname{soc}=\bigoplus_{b} I(b)^{d_{a b}^{\prime}} .
$$

Note that

$$
f_{a} d_{a b}=d_{a b}^{\prime} f_{b},
$$

where $f_{a}=\operatorname{dim}_{k}$ End $P(a)=\operatorname{dim}_{k}$ End $I(a)$, and similarly $f_{b}$. [This follows from the fact that we have $d_{a b}^{\prime}=\left.\right|_{\text {End } P(b)} \operatorname{Irr}(P(a), P(b)) \mid$, and

$$
\left.d_{a b}=\left|\operatorname{Irr}(P(a), P(b))_{\text {End } P(a)}\right| .\right]
$$

2. The theorem of Brenner-Butler. Let $T_{A}$ be a tilting module, with $\operatorname{End}\left(T_{A}\right)=B$. We consider the following functors $F, F^{\prime}: \mathfrak{\Re}_{A} \rightarrow \mathfrak{T}_{B}$ defined by

$$
F\left(M_{A}\right)=\operatorname{Hom}_{A}(T, M), \quad F^{\prime}\left(M_{A}\right)=\operatorname{Ext}_{A}^{1}(T, M),
$$


and $G, G^{\prime}: \Re_{B} \rightarrow \Re_{A}$ defined by

$$
G\left(N_{B}\right)=N_{B} \otimes_{B} T, \quad G^{\prime}\left(N_{B}\right)=\operatorname{Tor}_{1}^{B}(N, T) .
$$

In $\Re_{A}$, the following two full subcategories will be of interest:

$$
\mathscr{F}=\left\{M_{A} \mid F\left(M_{A}\right)=0\right\}, \quad \mathcal{T}=\left\{M_{A} \mid F^{\prime}\left(M_{A}\right)=0\right\} .
$$

Note that $\mathscr{F}=\mathscr{F}\left(T_{A}\right)$, and we will see below that also $\mathcal{T}=\mathcal{T}\left(T_{A}\right)$. Similarly, in $\mathfrak{T}_{B}$, we are interested in

$$
\mathfrak{X}=\left\{N_{B} \mid G\left(N_{B}\right)=0\right\}, \quad \mathcal{Y}=\left\{N_{B} \mid G^{\prime}\left(N_{B}\right)=0\right\} .
$$

(2.1) Theorem. Let $T_{A}$ be a tilting module with $\operatorname{End}\left(T_{A}\right)=B$. Then also ${ }_{B} T$ is a tilting module, and $A=\operatorname{End}\left({ }_{B} T\right)$, canonically. Also the functors $F, F^{\prime}, G, G^{\prime}$ defined above satisfy

$$
G^{\prime} F=G F^{\prime}=0, \quad F^{\prime} G=F G^{\prime}=0 .
$$

The categories $\mathcal{T}=\mathcal{T}\left(T_{A}\right)$ and $\mathcal{Y}$ are equivalent under the restrictions of the functors $F$ and $G$ (these restrictions are mutually inverse to each other), and similarly, the categories $\mathscr{F}=\mathscr{F}\left(T_{A}\right)$ and $\mathcal{X}$ are equivalent under the restrictions of $F^{\prime}$ and $G^{\prime}$ (again, these restrictions are mutually inverse to each other).

Proof. We need some additional notation. The functor $F$ is right adjoint to the functor $G$, thus there are canonical natural transformations

$$
t: G F \rightarrow \mathrm{id}_{\mathscr{N}_{A}}, \quad s: \mathrm{id}_{\Re_{B}} \rightarrow F G .
$$

(a) $\mathscr{T} \cap \mathscr{F}$ contains only the zero module.

Proof. The definition of a tilting module gives an exact sequence

$$
0 \rightarrow A_{A} \rightarrow T^{\prime} \rightarrow T^{\prime \prime} \rightarrow 0
$$

with $T^{\prime}, T^{\prime \prime} \in \operatorname{add}(T)$. Applying to this sequence the functor $\operatorname{Hom}_{A}\left(-, M_{A}\right)$, we obtain an exact sequence

$$
\operatorname{Hom}\left(T^{\prime}, M\right) \rightarrow \operatorname{Hom}\left(A_{A}, M\right) \rightarrow \operatorname{Ext}^{1}\left(T^{\prime \prime}, M\right) .
$$

If $M_{A} \in \mathcal{T} \cap \mathscr{F}$, then $\operatorname{Hom}(T, M)=0$, and $\operatorname{Ext}^{1}(T, M)=0$, thus also for $T^{\prime}, T^{\prime \prime} \in$ $\operatorname{add}(T)$, we have $\operatorname{Hom}\left(T^{\prime}, M\right)=0, \operatorname{Ext}^{\mathrm{l}}\left(T^{\prime \prime}, M\right)=0$, and therefore $M \approx$ $\operatorname{Hom}\left(A_{A}, M\right)=0$.

(b) $\sigma\left(T_{A}\right) \subseteq \sigma_{\text {. }}$

Proof. A surjective map $\oplus T_{A} \rightarrow M_{A}$ gives rise to a surjective map $F^{\prime}\left(\oplus T_{A}\right) \rightarrow$ $F^{\prime}\left(M_{A}\right)$, since p.d. $T_{A} \leqslant 1$. However $F^{\prime}\left(\oplus T_{A}\right)=\bigoplus F^{\prime}\left(T_{A}\right)=0$, again using that $T_{A}$ is a tilting module.

(c) For any $M_{A}$, the cokernel $\operatorname{Cok}\left(t_{M}\right)$ of the map $t_{M}: G F(M) \rightarrow M$ belongs to $\mathscr{F}$. If $M_{A} \in \mathcal{T}$, then $t_{M}$ is an isomorphism.

Proof. We factorize $t_{M}$ through its image, say:

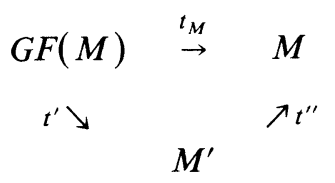


Now $F\left(t_{M}\right)$ is split epi (by abstract nonsense on adjoint functors); thus $F\left(t^{\prime \prime}\right)$ is surjective. Apply $F$ to the exact sequence

$$
0 \rightarrow M^{\prime} \stackrel{t^{\prime \prime}}{\rightarrow} M \rightarrow M^{\prime \prime} \rightarrow 0
$$

with $M^{\prime \prime}=\operatorname{Cok}\left(t^{\prime \prime}\right)=\operatorname{Cok}\left(t_{M}\right)$, we obtain an exact sequence

$$
0 \rightarrow F\left(M^{\prime}\right) \stackrel{F\left(t^{\prime \prime}\right)}{\rightarrow} F(M) \rightarrow F\left(M^{\prime \prime}\right) \rightarrow F^{\prime}\left(M^{\prime}\right) \rightarrow F^{\prime}(M) \rightarrow F^{\prime}\left(M^{\prime \prime}\right) \rightarrow 0 .
$$

Now $G F(M)=\operatorname{Hom}_{A}(T, M) \otimes_{B} T_{A}$ is clearly generated by $T_{A}$, thus also the epimorphic image $M^{\prime}$ of $G F(M)$; thus $M^{\prime} \in \mathcal{T}\left(T_{A}\right) \subseteq \mathcal{T}$, and therefore $F^{\prime}\left(M^{\prime}\right)=0$. As a consequence $F\left(M^{\prime \prime}\right)=0$.

Assume now $M \in \mathcal{T}$. The epimorphism $F^{\prime}(M) \rightarrow F^{\prime}\left(M^{\prime \prime}\right)$ shows that also $M^{\prime \prime} \in \mathcal{T}$; thus $M^{\prime \prime}=0$ according to (a). Thus $t_{M}$ is surjective. In particular we see that $M_{A}$ can be generated by $T_{A}$.

Let $f_{1}, \ldots, f_{n}$ be a basis of $\operatorname{Hom}(T, M)$, and consider the map $f=\left(f_{i}\right): \bigoplus_{i=1}^{n} T \rightarrow M$. Then $f$ is surjective, but also $\operatorname{Hom}(T, f)$ is surjective. Let $K=\operatorname{Ker} f$; thus we have the exact sequence

$$
0 \rightarrow K \rightarrow \bigoplus_{i=1}^{n} T \stackrel{f}{\rightarrow} M \rightarrow 0,
$$

and application of $\operatorname{Hom}(T,-)$ shows that $\operatorname{Ext}^{1}(T, K)=0$. In particular, we can apply the previous consideration also to $K$ (instead of $M$ ), and obtain a surjective map from a direct sum of copies of $T$ onto $K$ which remains surjective when applying $\operatorname{Hom}(T,-)$. Thus we have an exact sequence

$$
T_{1} \rightarrow T_{0} \rightarrow M \rightarrow 0
$$

with $T_{0}, T_{1}$ direct sums of copies of $T$, such that

$$
F\left(T_{1}\right) \rightarrow F\left(T_{0}\right) \rightarrow F(M) \rightarrow 0
$$

is again exact. If we now apply $G$, we again obtain an exact sequence, and we consider in addition the natural transformation $t$ :

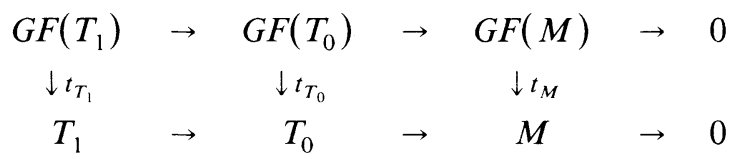

But clearly $t_{T}$ is an isomorphism, since $B=\operatorname{End}(T)$, and therefore also $t_{T_{1}}$ and $t_{T_{2}}$ are isomorphisms; thus $t_{M}$ is an isomorphism.

(d) For any $M_{A}$, we have $F\left(M_{A}\right) \in \mathcal{Y}$.

Proof. Choose a basis $f_{1}, \ldots, f_{n}$ in $\operatorname{Hom}(T, M)$ and consider the map $f=\left(f_{i}\right)$ : $\oplus T \rightarrow M$. It is not necessarily surjective, however $F(f)$ will be surjective. Let $K$ be the kernel of $f$, and $M^{\prime}$ the image, and note that the inclusion $M^{\prime} \hookrightarrow M$ gives rise to an isomorphism under $F$. Now the exact sequence

$$
0 \rightarrow K \rightarrow \oplus T \rightarrow M^{\prime} \rightarrow 0
$$

goes under $F$ to an exact sequence

$$
0 \rightarrow F(K) \rightarrow F(\oplus T) \rightarrow F\left(M^{\prime}\right) \rightarrow 0,
$$


and tensoring with ${ }_{B} T$ gives the upper exact row of:

$$
\begin{aligned}
& 0 \rightarrow \operatorname{Tor}_{1}^{B}\left(F\left(M^{\prime}\right), T\right) \rightarrow G F(K) \rightarrow G F(\oplus T) \rightarrow G F\left(M^{\prime}\right) \rightarrow 0 \\
& \downarrow t_{K} \quad \downarrow t_{\oplus T} \quad \downarrow t_{M} \\
& 0 \quad \rightarrow \quad K \quad \rightarrow \quad \oplus T \quad \rightarrow \quad M^{\prime} \quad \rightarrow 0
\end{aligned}
$$

Here we use the fact that $\operatorname{Tor}_{1}^{B}(F(\oplus T), T)=0$, since $F(\oplus T)$ is a free module. Now $M^{\prime}$ belongs to $\mathcal{T}$, thus, as we have seen above, also $K$ belongs to $\mathcal{T}$, therefore all three maps $t_{K}, t_{\oplus T}, t_{M^{\prime}}$ are isomorphisms. We conclude that $\operatorname{Tor}_{1}^{B}(F(M), T)=$ $\operatorname{Tor}_{1}^{B}\left(F\left(M^{\prime}\right), T\right)=0$.

(e) We have $\operatorname{End}\left({ }_{B} T\right)=A$, canonically.

Proof. There is a canonical isomorphism

$$
\begin{aligned}
\operatorname{Hom}_{B}\left({ }_{B} T,{ }_{B} T\right) & \cong \operatorname{Hom}_{B}\left({ }_{B} T,{ }_{B} T \otimes_{A} A\right) \\
& \cong \operatorname{Hom}_{B}\left({ }_{B} T, \operatorname{Hom}_{k}\left(\operatorname{Hom}_{k}\left({ }_{B} T \otimes_{A} A, k\right), k\right)\right) \\
& \cong \operatorname{Hom}_{k}\left(\operatorname{Hom}_{k}\left({ }_{B} T \otimes_{A} A, k\right) \otimes_{B} T, k\right) \\
& \cong \operatorname{Hom}_{k}\left(\operatorname{Hom}_{A}\left({ }_{B} T_{A}, \operatorname{Hom}\left({ }_{A} A, k\right)\right) \otimes_{B} T, k\right) \cong \operatorname{DGFD}\left({ }_{A} A\right),
\end{aligned}
$$

let us denote it by $\alpha$. Also, there is the natural map

$$
t=t_{D\left(C_{A} A\right)}: G F D\left({ }_{A} A\right) \rightarrow D\left({ }_{A} A\right)
$$

which is an isomorphism, since $D\left({ }_{A} A\right)$ is injective and therefore belongs to $\mathcal{T}$. Finally, let $\rho: A \rightarrow \operatorname{End}\left({ }_{B} T\right)$ be the multiplication map $a \mapsto(x \mapsto x a)$. Then one checks that the diagram

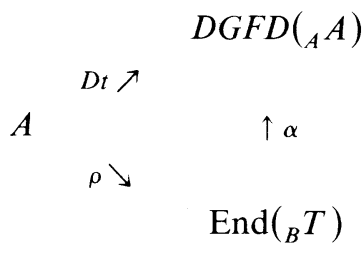

commutes; thus, since both $\alpha$ and $D t$ are bijective, also $\rho$ is bijective.

(f) If $N_{B} \in \mathcal{Y}$, then $s_{N}$ is an isomorphism.

Proof. Choose an exact sequence

$$
0 \rightarrow L_{B} \rightarrow P_{B} \rightarrow N_{B} \rightarrow 0
$$

with $P_{B}$ projective. Application of $G$ gives again an exact sequence, since $\operatorname{Tor}_{1}^{B}(N, T)$ $=0$, namely

$$
0 \rightarrow L_{B} \otimes_{B} T \rightarrow P_{B} \otimes_{B} T \rightarrow N_{B} \otimes_{B} T \rightarrow 0 .
$$

Now $L_{B} \otimes_{B} T_{A}$ is generated by $T_{A}$; thus in $\mathcal{T}$, therefore the application of $F$ again leads to an exact sequence

$$
0 \rightarrow F G(L) \rightarrow F G(P) \rightarrow F G(N) \rightarrow 0 .
$$

Similarly, let

$$
0 \rightarrow L^{\prime} \stackrel{m}{\rightarrow} P^{\prime} \rightarrow L \rightarrow 0
$$


be exact, with $P^{\prime}$ projective. Application of $G$ leads to the exact sequence

$$
L_{B}^{\prime} \otimes_{B} T \stackrel{m \otimes 1}{\rightarrow} P_{B}^{\prime} \otimes_{B} T \rightarrow L_{B} \otimes_{B} T \rightarrow 0 .
$$

Let $K$ be the image of $m \otimes 1$. Since $K$ is an epimorphic image of $L_{B}^{\prime} \otimes_{B} T$, we see that $K$ is generated by $T$; thus $K$ belongs to $\mathcal{T}$, and therefore the exact sequence

$$
0 \rightarrow K \rightarrow P_{B}^{\prime} \otimes_{B} T \rightarrow L_{B} \otimes_{B} T \rightarrow 0
$$

goes under $\operatorname{Hom}(T,-)$ to an exact sequence:

$$
0 \rightarrow \operatorname{Hom}(T, K) \rightarrow \underbrace{\operatorname{Hom}\left(T, P_{B}^{\prime} \otimes_{B} T_{A}\right)}_{=F G\left(P^{\prime}\right)} \rightarrow \underbrace{\operatorname{Hom}\left(T, L_{B} \otimes_{B} T_{A}\right)}_{=F G(L)} \rightarrow 0
$$

Altogether we see that we have the following commutative diagram with exact rows:

$$
\begin{array}{ccccccc}
F G\left(P^{\prime}\right) & \rightarrow & F G(P) & \rightarrow & F G(N) & \rightarrow & 0 \\
\uparrow s_{P^{\prime}} & & \uparrow s_{P} & & \uparrow s_{N} & & \\
P^{\prime} & \rightarrow & P & \rightarrow & N & \rightarrow & 0
\end{array}
$$

Now clearly $s_{B_{B}}$ is an isomorphism, since $A=\operatorname{End}\left({ }_{B} T\right)$, therefore also $s_{P^{\prime}}$ and $s_{P}$ are isomorphisms, due to the fact that $P$ and $P^{\prime}$ are projective. Thus $s_{N}$ is an isomorphism.

(g) $G F^{\prime}=0$, and, if $M_{A} \in \mathscr{F}$, then $M_{A} \approx G^{\prime} F^{\prime}(M)$.

Proof. First, let $M_{A}$ be arbitrary, and

$$
0 \rightarrow M_{A} \rightarrow I_{A} \rightarrow Q_{A} \rightarrow 0
$$

exact, with $I_{A}$ injective. We apply $F$ and obtain an exact sequence

$$
0 \rightarrow F(M) \rightarrow F(I) \rightarrow F(Q) \rightarrow \operatorname{Ext}^{1}(T, M) \rightarrow 0,
$$

the last zero due to the fact that $I$ is injective. Application of $G$ leads to:

$$
\begin{array}{ccccc}
G F(I) & \rightarrow & G F(Q) & \rightarrow & \operatorname{Ext}^{1}(T, M) \otimes_{B} T \rightarrow 0 \\
\downarrow t_{I} & & \downarrow t_{Q} & & \\
I & \rightarrow & Q & \rightarrow & 0
\end{array}
$$

Now $I$ belongs to $\mathscr{T}$, since $I$ is injective, and since p.d. $T_{A} \leqslant 1$, we know that $\mathcal{T}$ is

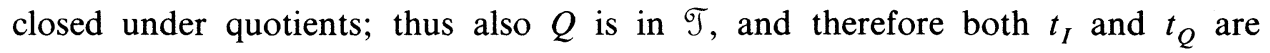
isomorphisms. This shows $G F^{\prime}(M)=0$. If now we have in addition $F(M)=0$, then the exact sequence

$$
0 \rightarrow F(I) \rightarrow F(Q) \rightarrow \operatorname{Ext}^{1}(T, M) \rightarrow 0
$$

gives under $G$ the upper exact sequence of

$$
\begin{aligned}
& 0 \rightarrow \operatorname{Tor}_{1}\left(\operatorname{Ext}^{1}(T, M), T\right) \rightarrow G F(I) \rightarrow G F(Q) \rightarrow 0 \\
& 0 \rightarrow M \quad \rightarrow \quad \begin{array}{lllll}
\downarrow t_{I} & & \downarrow t_{Q} & & \\
& \rightarrow & Q & \rightarrow & 0
\end{array}
\end{aligned}
$$

where we also use that $\operatorname{Tor}_{1}(F(Q), T)=G^{\prime} F(Q)=0$. We conclude that there exists a canonical isomorphism $G^{\prime} F^{\prime}(M) \rightarrow M$.

(h) p.d. ${ }_{B} T \leqslant 1$.

Proof. Apply to the exact sequence

$$
0 \rightarrow A_{A} \rightarrow T^{\prime} \rightarrow T^{\prime \prime} \rightarrow 0
$$


with $T^{\prime}, T^{\prime \prime} \in \operatorname{add} T$ the functor $\operatorname{Hom}_{A}(-, T)$. Since $\operatorname{Ext}^{1}\left(T_{A}, T_{A}\right)=0$, we obtain the exact sequence

$$
0 \rightarrow \operatorname{Hom}_{A}\left(T_{A}^{\prime \prime},{ }_{B} T_{A}\right) \rightarrow \operatorname{Hom}_{A}\left(T_{A}^{\prime},{ }_{B} T_{A}\right) \rightarrow \operatorname{Hom}_{A}\left(A_{A},{ }_{B} T_{A}\right) \rightarrow 0 .
$$

Now $\operatorname{Hom}_{A}\left(A_{A},{ }_{B} T_{A}\right) \approx_{B} T$, and the $B$-modules $\operatorname{Hom}_{A}\left(T_{A}^{\prime},{ }_{B} T_{A}\right), \operatorname{Hom}_{A}\left(T_{A}^{\prime \prime}{ }_{B} T_{A}\right)$ both are projective, since $T^{\prime}, T^{\prime \prime} \in$ add $T_{A}$.

(i) There is an exact sequence $0 \rightarrow{ }_{B} B \rightarrow{ }_{B} X^{\prime} \rightarrow{ }_{B} X^{\prime \prime} \rightarrow 0$ with $X^{\prime}, X^{\prime \prime} \in \operatorname{add}_{B} T$.

Proof. Start with a projective resolution

$$
0 \rightarrow P_{A}^{\prime \prime} \rightarrow P_{A}^{\prime} \rightarrow T_{A} \rightarrow 0
$$

and apply $\operatorname{Hom}_{A}\left(-,{ }_{B} T_{A}\right)$. Since $\operatorname{Ext}^{1}\left(T_{A}, T_{A}\right)=0$, we obtain an exact sequence

$$
0 \rightarrow \operatorname{Hom}_{A}\left(T_{A},{ }_{B} T_{A}\right) \rightarrow \operatorname{Hom}_{A}\left(P_{A}^{\prime},{ }_{B} T_{A}\right) \rightarrow \operatorname{Hom}_{A}\left(P_{A}^{\prime \prime},{ }_{B} T_{A}\right) \rightarrow 0 .
$$

Here, $\operatorname{Hom}_{A}\left(T_{A},{ }_{B} T_{A}\right) \approx_{B} B$, and $\operatorname{Hom}_{A}\left(P_{A}^{\prime},{ }_{B} T_{A}\right), \operatorname{Hom}_{A}\left(P_{A}^{\prime \prime},{ }_{B} T_{A}\right)$ both belong to add $_{B} T$, since $P_{A}^{\prime}, P_{A}^{\prime \prime} \in$ add $A_{A}$.

(j) $F G^{\prime}=0$, and, if $N_{B} \in \mathcal{X}$, then $N_{B} \approx F^{\prime} G^{\prime}(N)$.

Proof. First, let $N_{B}$ be arbitrary, and let

$$
0 \rightarrow K_{B} \rightarrow P_{B} \rightarrow N_{B} \rightarrow 0
$$

be exact with $P_{B}$ projective. Tensoring with ${ }_{B} T$ gives

$$
0 \rightarrow \operatorname{Tor}_{1}^{B}(N, T) \rightarrow G(K) \rightarrow G(P) \rightarrow G(N) \rightarrow 0 ;
$$

application of $F$ gives:



Note that both $s_{K}$ and $s_{P}$ are isomorphisms. Namely, p.d. ${ }_{B} T \leqslant 1$ implies that $\operatorname{Tor}_{1}^{B}\left(-,{ }_{B} T\right)$ is left exact; thus with $P$ also $K$ belongs to $\mathcal{Y}$. It follows that $F G^{\prime}(N)=0$. In case $G(N)=0$, we have the exact sequence

$$
0 \rightarrow G^{\prime}(N) \rightarrow G(K) \rightarrow G(P) \rightarrow 0 .
$$

Application of $F$ leads to the upper exact sequence of

$$
\begin{aligned}
& 0 \rightarrow F G(K) \rightarrow F G(P) \rightarrow \operatorname{Ext}_{A}^{1}\left(T, G^{\prime}(N)\right) \rightarrow 0 \\
& \begin{array}{llllllll} 
& \uparrow s_{K} & & \uparrow s_{P} \\
0 & K & \rightarrow & P & \rightarrow & N & \rightarrow & 0
\end{array}
\end{aligned}
$$

where the last zero in the first row comes from the fact that $F^{\prime} G=0$. Since we have noted that $s_{K}$ and $s_{P}$ are isomorphisms, we conclude that there exists an isomorphism $N \rightarrow F^{\prime} G^{\prime}(N)$.

(k) $\operatorname{Ext}^{1}\left({ }_{B} T,{ }_{B} T\right)=0$.

Proof. We will show that $\operatorname{Ext}^{1}\left(D\left({ }_{B} T\right), D\left({ }_{B} T\right)\right)=0$. Now

$$
\begin{aligned}
D\left({ }_{B} T\right) & =\operatorname{Hom}_{k}\left({ }_{B} T, k\right) \cong \operatorname{Hom}_{k}\left({ }_{B} T_{A} \otimes_{A} A, k\right) \\
& \cong \operatorname{Hom}_{A}\left(T, \operatorname{Hom}\left({ }_{A} A, k\right)\right)=F\left(D\left({ }_{A} A\right)\right) ;
\end{aligned}
$$

thus $D\left({ }_{B} T\right)$ belongs to $Y$. Let

$$
0 \rightarrow D\left({ }_{B} T\right) \rightarrow N_{B} \rightarrow D\left({ }_{B} T\right) \rightarrow 0
$$


be an exact sequence. Since $D\left({ }_{B} T\right)$ belongs to $\mathcal{Y}$, and $\mathcal{Y}$ is closed under extensions, also $N_{B}$ belongs to $\mathcal{Y}$. Thus, under $G$ this exact sequence goes to the sequence

$$
0 \rightarrow D\left({ }_{B} T\right) \otimes_{B} T \rightarrow N_{B} \otimes_{B} T \rightarrow D\left({ }_{B} T\right) \otimes_{B} T \rightarrow 0
$$

which also is exact, since $\operatorname{Tor}_{1}^{B}\left(D\left({ }_{B} T\right),{ }_{B} T\right)=0$. However, $D\left({ }_{B} T\right) \otimes_{B} T_{A} \approx D\left({ }_{A} A\right)$ is injective, thus the sequence splits. Applying $F$, we obtain back the given exact sequence, which then also has to split.

This finishes the proof of the theorem: The assertions $(\mathrm{h}),(\mathrm{j}),(\mathrm{k})$ show that ${ }_{B} T$ is a tilting module. We have $G^{\prime} F=0$ according to (d), and $G F^{\prime}=0, F G^{\prime}=0$ according to (g) and (j). Also, $F^{\prime} G=0$, since for any $B$-module $N_{B}$, the module $G\left(N_{B}\right)$ clearly is generated by $T_{A}$, thus belongs to $\mathscr{T}\left(T_{A}\right)$, and $\mathcal{T}\left(T_{A}\right) \subseteq \mathcal{T}$ according to (b). Since by (c), any $M_{A} \in \mathcal{T}$ is generated by $T_{A}$, we actually have $\mathcal{T}\left(T_{A}\right)=\mathcal{T}$. Finally, the equivalence of $\mathcal{T}$ and $\mathscr{Y}$ has been shown in (c) and (f), whereas the equivalence of $\mathscr{F}$ and $\mathscr{X}$ is shown in $(\mathrm{g})$ and $(\mathrm{j})$.

Note that the proofs in (b), (c), and (e) are directly taken from the paper [6] of Brenner and Butler.

REMARK 1. Given a tilting module $T_{A}$, and any projective module $P_{A}$, there is an exact sequence of the form

$$
0 \rightarrow P_{A} \rightarrow T^{\prime} \rightarrow T^{\prime \prime} \rightarrow 0
$$

with $T^{\prime}, T^{\prime \prime} \in \operatorname{add}\left(T_{A}\right)$.

This follows directly as in the proof (i) above, starting with a projective resolution of a suitable module ${ }_{B} N$ in $\operatorname{add}\left({ }_{B} T\right)$.

Conversely, instead of property (3) of a tilting module, it is sufficient to assume that there exists an exact sequence

$$
0 \rightarrow P_{A} \rightarrow T^{\prime} \rightarrow T^{\prime \prime} \rightarrow 0
$$

with $T^{\prime}, T^{\prime \prime} \in$ add $T_{A}$, such that any indecomposable projective module is a direct summand of $P_{A}$.

Proof. In this case, $\operatorname{Hom}\left(P_{A}, M\right)=0$ implies $M=0$, this is used in (a), similarly, in (h) one obtains p.d. ${ }_{B} \operatorname{Hom}\left(P_{A},{ }_{B} T_{A}\right)$ and therefore also p.d. ${ }_{B} T \leqslant 1$, since ${ }_{B} T \in$ $\operatorname{add}_{B} \operatorname{Hom}\left(P_{A},{ }_{B} T_{A}\right)$. It follows from (h), (i), (k) that ${ }_{B} T$ is a tilting module, and (e) shows $A=\operatorname{End}\left(T_{B}\right)$. Thus also ${ }_{A} T$ is a tilting module.

(2.2) If we consider left $B$-modules instead of right $B$-modules, the result becomes fully symmetric. Namely, we will see that $\mathcal{X}$ is the dual of $\mathscr{F}\left({ }_{B} T\right)$, and $\mathcal{Y}$ the dual of $\widetilde{T}\left({ }_{B} T\right)$. We need the following lemma:

LEMma. If p.d. ${ }_{B} T \leqslant 1$, then there are isomorphisms

$$
D\left({ }_{B} N\right) \otimes_{B} T_{A} \approx D \operatorname{Hom}_{B}\left({ }_{B} T_{A},{ }_{B} T\right),
$$

and

$$
\operatorname{Tor}_{1}^{B}\left(D\left({ }_{B} N\right),{ }_{B} T_{A}\right) \approx D \operatorname{Ext}_{B}^{1}\left({ }_{B} T_{A},{ }_{B} N\right),
$$

both natural in ${ }_{B} N$.

Proof. Take a projective resolution

$$
0 \rightarrow{ }_{B} P^{\prime \prime} \rightarrow{ }_{B} P^{\prime} \rightarrow{ }_{B} T \rightarrow 0
$$


and apply functors $D \operatorname{Hom}_{B}\left(-,{ }_{B} N\right)$ and $D\left({ }_{B} N\right) \otimes_{B}-$, where ${ }_{B} N$ is some $B$-module. We obtain the following two exact sequences:

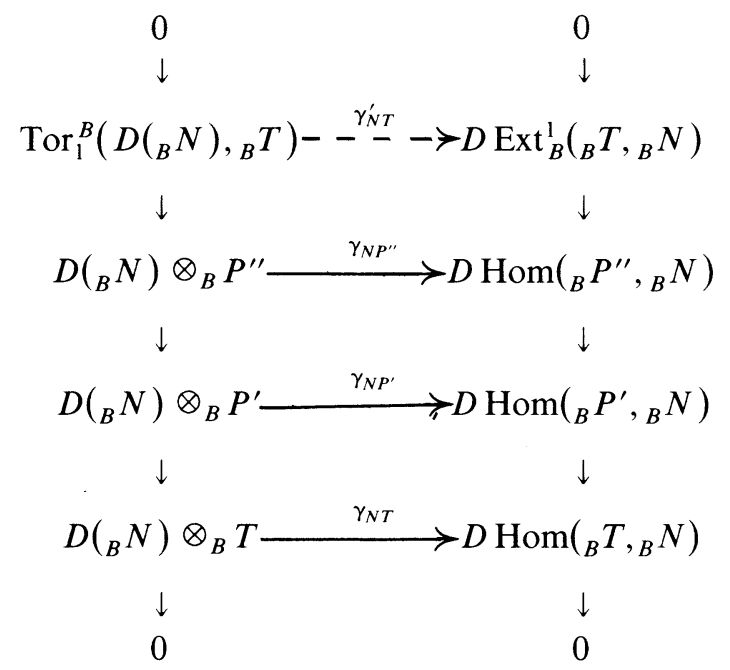

The natural transformation $\gamma_{N U}: D\left({ }_{B} N\right) \otimes_{B} U \rightarrow D \operatorname{Hom}\left({ }_{B} U,{ }_{B} N\right)$ is defined as

$$
\left[\gamma_{N U}(\varphi \otimes u)\right](f)=\varphi(u f)
$$

where $\varphi \in D\left({ }_{B} N\right), u \in U, f \in \operatorname{Hom}\left({ }_{B} U,{ }_{B} N\right)$. Clearly $\gamma_{N U}$ is an isomorphism for $U={ }_{B} B$, and therefore for $U$ projective. Thus $\gamma_{N P^{\prime}}$ and $\gamma_{N P^{\prime \prime}}$ are isomorphisms. This shows that also $\gamma_{N T}$ is an isomorphism, and that the natural isomorphism $\gamma_{N T}^{\prime}$ is defined.

Corollary $1 . D \mathcal{Y}=\mathcal{T}\left({ }_{B} T\right), D \mathscr{X}=\mathscr{F}\left({ }_{B} T\right)$.

COROLlaRY 2. The restrictions of the functors

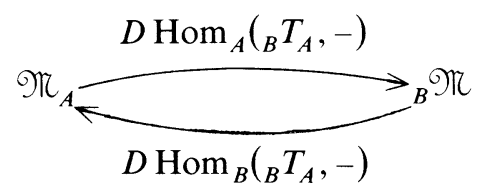

define a duality between $\mathcal{T}\left(T_{A}\right)$ and $\mathcal{T}\left({ }_{B} T\right)$. Similarly, the restrictions of the functors

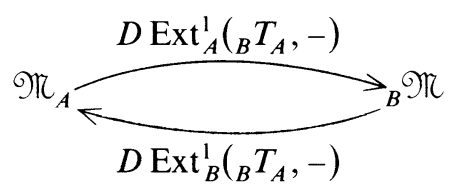

define a duality between $\mathscr{F}\left(T_{A}\right)$ and $\mathscr{F}\left({ }_{B} T\right)$.

Proof. Both corollaries are direct consequences of (2.1) using the previous lemma.

(2.3) For the further investigation, it will be of interest to study the relation between the $B$-modules of the forms $F(I)$ and $F^{\prime}(P)$, where $I$ is indecomposable injective and $P$ indecomposable projective. 
Connecting lemma. Let $T_{A}$ be a tilting module, with $B=\operatorname{End}\left(T_{A}\right)$. Let $I_{A}$ be injective, $P_{A}$ projective with $\operatorname{soc} I_{A} \approx P_{A} / \operatorname{rad} P_{A}$. Then $\tau^{-1} \operatorname{Hom}_{A}\left({ }_{B} T_{A}, I_{A}\right)=$ $\operatorname{Ext}_{A}^{1}\left({ }_{B} T_{A}, P_{A}\right)$.

Proof. Since $T_{A}$ is a tilting module, there is an exact sequence

$$
0 \rightarrow P_{A} \rightarrow T^{\prime} \stackrel{\pi}{\rightarrow} T^{\prime \prime} \rightarrow 0
$$

where $T^{\prime}, T^{\prime \prime} \in \operatorname{add}\left(T_{A}\right)$ (see the remark after Theorem 2.1). Under $\operatorname{Hom}_{A}\left(-, T_{A}\right)$, we obtain the exact sequence

$$
0 \rightarrow \operatorname{Hom}_{A}\left(T^{\prime \prime}, T\right) \stackrel{\operatorname{Hom}_{A}(\pi, T)}{\rightarrow} \operatorname{Hom}_{A}\left(T^{\prime}, T\right) \rightarrow \operatorname{Hom}_{A}\left(P_{A},{ }_{B} T_{A}\right) \rightarrow 0
$$

and this is a projective resolution of $\operatorname{Hom}_{A}\left(P_{A},{ }_{B} T_{A}\right)$, with $\operatorname{Hom}_{A}\left(T^{\prime \prime}, T^{\prime}\right)$ minimal. Now the transpose of $\operatorname{Hom}_{A}(\pi, T)$ clearly is $\operatorname{Hom}_{A}(T, \pi)$, and we have the exact sequence.

$$
\operatorname{Hom}_{A}\left(T, T^{\prime}\right) \stackrel{\operatorname{Hom}_{A}(T, \pi)}{\rightarrow} \operatorname{Hom}_{A}\left(T, T^{\prime \prime}\right) \rightarrow \operatorname{Ext}_{A}^{1}(T, P) \rightarrow 0
$$

which stems from the sequence (*) by applying $\operatorname{Hom}_{A}\left(T_{A},-\right)$ and using $\operatorname{Ext}_{A}^{1}\left(T_{A}, T_{A}\right)$ $=0$. Thus $\operatorname{Ext}_{A}^{1}\left({ }_{B} T_{A}, P_{A}\right)=\operatorname{Tr} \operatorname{Hom}_{A}\left(P_{A},{ }_{B} T_{A}\right)$. However, since $P_{A} / \operatorname{rad} P_{A} \approx \operatorname{soc} I_{A}$, there is a canonical isomorphism $\operatorname{Hom}_{A}\left(P_{A},{ }_{B} T_{A}\right) \approx D \operatorname{Hom}_{A}\left({ }_{B} T_{A}, I_{A}\right)$; thus

$$
\begin{aligned}
\tau^{-1} \operatorname{Hom}_{A}\left({ }_{B} T_{A}, I_{A}\right) & =\operatorname{Tr} D \operatorname{Hom}_{A}\left({ }_{B} T_{A}, I_{A}\right) \approx \operatorname{Tr} \operatorname{Hom}_{A}\left(P_{A},{ }_{B} T_{A}\right) \\
& =\operatorname{Ext}_{A}^{1}\left({ }_{B} T_{A}, P_{A}\right) .
\end{aligned}
$$

REMARK 1. Let us reformulate the connecting lemma in the case of considering left $B$-modules:

Let $T_{A}$ be a tilting module, $\operatorname{End}\left(T_{A}\right)=B$. If $I_{A}$ is injective, $P_{A}$ projective, with $\operatorname{soc} I_{A} \approx P_{A} / \operatorname{rad} P_{A}$, then

$$
\tau D \operatorname{Hom}_{A}\left({ }_{B} T_{A}, I_{A}\right)=D \operatorname{Ext}_{A}^{1}\left({ }_{B} T_{A}, P_{A}\right) .
$$

If ${ }_{B} J$ is injective, ${ }_{B} Q$ projective, with $\operatorname{soc}_{B} J \approx_{B} Q / \operatorname{rad}_{B} Q$, then

$$
\tau D \operatorname{Hom}_{B}\left({ }_{B} T_{A},{ }_{B} J\right)=D \operatorname{Ext}_{B}^{1}\left({ }_{B} T_{A},{ }_{B} Q\right) .
$$

Proof. For the first formula, we apply $D$ to the previous equality, noting that $\tau D\left(N_{B}\right)=D \tau^{-1} N_{B}$ for any module $N_{B}$. The second formula follows by left-right symmetry.

REMARK 2. We obtain a further reformulation of the connecting lemma as follows:

Again, assume $T_{A}$ to be a tilting module, $\operatorname{End}\left(T_{A}\right)=B$. Let $T_{A}^{\prime}$ be in add $T_{A}$; thus $Q_{B}=F\left(T_{A}^{\prime}\right)$ is projective. Let $J_{B}$ be injective with $\operatorname{soc} J_{B}=Q_{B} / \operatorname{rad} Q_{B}$. Then $\tau T_{A}^{\prime}=$ $\operatorname{Tor}_{1}^{B}\left(J_{B},{ }_{B} T_{A}\right)$.

Proof. We have

$$
\begin{aligned}
\operatorname{Tor}_{1}^{B}\left(J_{B},{ }_{B} T_{A}\right) & \approx D \operatorname{Ext}_{B}^{1}\left({ }_{B} T_{A}, D\left(J_{B}\right)\right) \\
& =\tau D \operatorname{Hom}_{B}\left({ }_{B} T_{A}, D\left(Q_{B}\right)\right) \approx \tau T_{A}^{\prime},
\end{aligned}
$$


using the second formula of Remark 1, and the isomorphism

$$
\begin{aligned}
D \operatorname{Hom}_{B}\left({ }_{B} T_{A}, D\left(Q_{B}\right)\right) & =D \operatorname{Hom}_{B}\left({ }_{B} T_{A}, \operatorname{Hom}_{k}\left(Q_{B}, k\right)\right) \\
& \approx D \operatorname{Hom}_{k}\left(Q_{B} \otimes_{B} T_{A}, k\right) \approx Q_{B} \otimes_{B} T_{A} \approx T_{A}^{\prime} .
\end{aligned}
$$

(2.4) We use the connecting lemma, in order to derive conditions on the injective $B$-modules.

Corollary. Let $T_{A}$ be a tilting module, $B=\operatorname{End}\left(T_{A}\right)$. Let $I_{A}$ be injective, $P_{A}$ projective, with $\operatorname{soc} I_{A}=P_{A} / \operatorname{rad} P_{A}$. If $P_{A} \in \operatorname{add} T_{A}$, then $\operatorname{Hom}_{A}\left({ }_{B} T_{A}, I_{A}\right)$ is injective. All injective B-modules which belong to $\mathcal{O}$ are of this form.

Proof. If $P_{A} \in \operatorname{add} T_{A}$, then $\operatorname{Ext}_{A}^{1}\left({ }_{B} T_{A}, P_{A}\right)=0$; thus by the connecting lemma, $\tau^{-1} \operatorname{Hom}_{A}\left({ }_{B} T_{A}, I_{A}\right)=0$. Conversely, let $\operatorname{Hom}_{A}\left({ }_{B} T_{A}, M_{A}\right)$ be injective. We can assume $M_{A}$ indecomposable, and in $\widetilde{T}$. Let $M_{A} \stackrel{\mu}{\hookrightarrow} I_{A}^{\prime}$ be an injective hull. Then $F(\mu)$ : $F(M) \rightarrow F\left(I^{\prime}\right)$ is mono again; thus a split mono, since we assume $F(M)$ to be injective. However, both $M_{A}$ and $I_{A}^{\prime}$ belong to $\mathcal{T}$; thus $\mu$ is isomorphic to $G F(\mu)$, and therefore is itself split mono. This shows that $M_{A}$ is injective, say $M_{A}=I(a)_{A}$. Let $P(a)_{A}$ be projective with $P(a) / \operatorname{rad} P(a)=\operatorname{soc} I(a)$. If $P(a)_{A}$ would not be in add $T$, then using the connecting lemma again, we would have $\tau^{-1} F I(a)=F^{\prime} P(a) \neq$ 0 , contrary to the assumption that $F I(a)$ is injective.

Corollary 2. Let $T_{A}$ be a tilting module, $B=\operatorname{End}\left(T_{A}\right)$. If both $P(a)$ and $I(a)$ are in add $T_{A}$, then $\operatorname{Hom}_{A}\left({ }_{B} T_{A}, I(a)_{A}\right)$ is projective and injective, and conversely every indecomposable module which is at the same time projective and injective is of this form.

Proof. The first assertion follows immediately from the previous corollary.

For the second assertion, let $N_{B}$ be indecomposable, projective and injective. Since $N_{B}$ is indecomposable and projective, it is of the form $N_{B}=\operatorname{Hom}_{A}\left({ }_{B} T_{A}, T(i)_{A}\right)$, for some direct summand $T(i)$ of $T_{A}$, in particular, $N_{B} \in \mathcal{Y}$. Since $N_{B}$ is also injective, it follows from the previous corollary that $N_{B}=\operatorname{Hom}_{A}\left({ }_{B} T_{A}, I(a)_{A}\right)$ for some $I(a)$ with $P(a) \in$ add $T_{A}$. Finally, since both $T(i)$ and $I(a)$ belong to $厅\left(T_{A}\right)$, and $\operatorname{Hom}_{A}\left({ }_{B} T_{A},-\right)$ induces an equivalence between $\mathcal{T}\left(T_{A}\right)$ and $\mathcal{Y}$, we see that $I(a)_{A} \approx T(i)_{A}$ also belongs to add $T_{A}$. This finishes the proof.

(2.5) Let us remark that $D \operatorname{Ext}_{A}^{1}\left(T_{A}, M_{A}\right)$ can always be identified with a factor group of $\operatorname{Hom}_{A}\left(M_{A}, \tau T_{A}\right)$, where $\tau$ is the Auslander-Reiten translation. In fact, in our situation, both groups coincide, according to the following lemma:

Lemma. If p.d. $T_{A} \leqslant 1$, then $D \operatorname{Ext}_{A}^{1}\left(T_{A}, M_{A}\right) \approx \operatorname{Hom}_{A}\left(M_{A}, \tau T_{A}\right)$.

Proof. Choose a minimal projective resolution

$$
0 \rightarrow P_{A}^{\prime \prime} \rightarrow P_{A}^{\prime} \rightarrow T_{A} \rightarrow 0
$$

of $T_{A}$. This gives an exact sequence

$$
\operatorname{Hom}\left(P^{\prime}, M\right) \rightarrow \operatorname{Hom}\left(P^{\prime \prime}, M\right) \rightarrow \operatorname{Ext}^{1}(T, M) \rightarrow 0 .
$$

By definition of $\operatorname{Tr} T_{A}$, we have $\operatorname{Tr} T_{A}=\operatorname{Ext}_{A}^{1}\left(T_{A}, A_{A}\right)$. Replacing $M_{A}$ by $A_{A}$, and dualizing, we obtain the exact sequence

$$
0 \rightarrow \tau T_{A} \rightarrow D \operatorname{Hom}\left(P_{A}^{\prime \prime}, A_{A}\right) \rightarrow D \operatorname{Hom}\left(P_{A}^{\prime}, A_{A}\right) .
$$


If we apply to this sequence $D \operatorname{Hom}_{A}\left(M_{A},-\right)$, we get the left exact column of the diagram

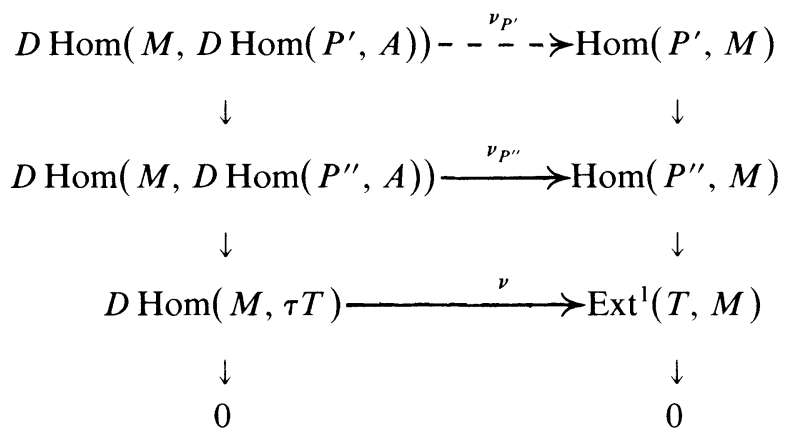

where $\nu$ is the natural transformation with $\nu_{X}$, for an $A$-module $X_{A}$, being

$$
\begin{aligned}
D \operatorname{Hom}_{A}\left(M, D \operatorname{Hom}_{A}(X, A)\right) & =D \operatorname{Hom}_{A}\left(M, \operatorname{Hom}_{k}\left(\operatorname{Hom}_{A}(X, A), k\right)\right) \\
& \approx D \operatorname{Hom}_{k}\left(M_{A} \otimes \operatorname{Hom}_{A}(X, A), k\right) \\
& =D D\left(M_{A} \otimes \operatorname{Hom}_{A}(X, A)\right) \\
& \approx M_{A} \otimes \operatorname{Hom}_{A}(X, A) \rightarrow \operatorname{Hom}_{A}(X, M),
\end{aligned}
$$

the last map being given by $m \otimes \varphi \mapsto(x \mapsto m \varphi(x))$, for $m \in M, \varphi \in \operatorname{Hom}_{A}(X, A)$, and $x \in X_{A}$. This last map, and therefore $\nu_{X}$, is an isomorphism for $X_{A}=A_{A}$. As a consequence, the same is true for $X_{A}$ projective. Since both $\nu_{P^{\prime}}, \nu_{P^{\prime \prime}}$ are isomorphisms, we can see that there is an isomorphism $D \operatorname{Hom}(M, \tau T) \approx \operatorname{Ext}^{1}(T, M)$.

COROLlary. If $T_{A}$ is a tilting module, then $\mathscr{F}\left(T_{A}\right)$ is the set of all right $A$-modules cogenerated by $\tau T_{A}$.

Proof. If $M_{A}$ is cogenerated by $\tau T_{A}$, say $M$ is embedded in $\bigoplus_{i=1}^{m} \tau T_{A}$, then $\operatorname{Hom}(T, M)=0, \operatorname{since} \operatorname{Hom}(T, \tau T)=\operatorname{Ext}^{\mathrm{l}}(T, T)=0$.

Conversely, let $M_{A} \in \mathscr{F}\left(T_{A}\right)$. Then there is $N_{B} \in \mathcal{X}$ with $M_{A}=\operatorname{Tor}_{1}^{B}\left(N_{B},{ }_{B} T_{A}\right) \approx$ $D \operatorname{Ext}_{B}^{1}\left({ }_{B} T_{A}, D\left(N_{B}\right)\right)$. Let $N_{B} \subseteq I_{B}$, with $I_{B}$ injective. The dual map $D\left(I_{B}\right) \rightarrow D\left(N_{B}\right)$ is surjective, so, since p.d. ${ }_{B} T \leqslant 1$, the induced map

$$
\operatorname{Ext}_{B}^{1}\left({ }_{B} T_{A}, D\left(I_{B}\right)\right) \rightarrow \operatorname{Ext}_{B}^{1}\left({ }_{B} T_{A}, D\left(N_{B}\right)\right)
$$

also is surjective. Dualizing, we obtain an injection

$$
M_{A} \approx D \operatorname{Ext}_{B}^{1}\left({ }_{B} T_{A}, D\left(N_{B}\right)\right) \rightarrow D \operatorname{Ext}_{B}^{1}\left({ }_{B} T_{A}, D\left(I_{B}\right)\right) .
$$

Now $D \operatorname{Ext}_{B}^{1}\left({ }_{B} T_{A}, D\left(I_{B}\right)\right)$ is a direct sum of modules of the form $D \operatorname{Ext}_{B}^{1}\left({ }_{B} T_{A},{ }_{B} P\right)$, with ${ }_{B} P$ indecomposable projective, thus $M_{A}$ is cogenerated by these types of modules. On the other hand, let ${ }_{B} I(i)=D \operatorname{Hom}_{A}\left({ }_{B} T_{A},\left(T_{i}\right)_{A}\right)$ where $T_{i}$ is an indecomposable direct summand of $T_{A}$. Since $\left(T_{i}\right)_{A} \in \mathcal{T}$, it follows from (2.2) that $\left(T_{i}\right)_{A} \approx D \operatorname{Hom}_{B}\left({ }_{B} T_{A},{ }_{B} I(i)\right)$. The connecting lemma shows that for $P(i)$, with $P(i) / \operatorname{rad} P(i) \approx \operatorname{soc} I(i)$, we have

$$
\tau D \operatorname{Hom}\left({ }_{B} T_{A},{ }_{B} I(i)\right) \approx D \operatorname{Ext}^{1}\left({ }_{B} T_{A},{ }_{B} P(i)\right) .
$$

Thus $\tau T_{i} \approx \tau D \operatorname{Hom}\left({ }_{B} T_{A},{ }_{B} I(i)\right) \approx D \operatorname{Ext}^{1}\left({ }_{B} T_{A},{ }_{B} P(i)\right)$, and therefore $M_{A}$ is cogenerated by the set of modules of the form $\tau T_{i}$, thus by $\tau T$. 
Corollary. $\mathcal{X}$ is the set of modules generated by $F^{\prime}\left(A_{A}\right)$.

(2.6) The tilting conditions of Brenner and Butler. This section will not be needed in the sequel. However, it seems to be of interest to verify that modules satisfying the "tilting conditions" $\left(T_{*}\right)$ or $\left(T_{*}^{\prime}\right)$ of Brenner and Butler [6] are tilting modules in the sense of this paper. These are the tilting conditions for the right $A$-module $T_{A}$ :

$\left(T_{1}\right)$ p.d. $T_{A} \leqslant 1$.

$\left(T_{2}\right) \operatorname{Ext}_{A}^{1}\left(T_{A}, T_{A}\right)=0$.

$\left(T_{3}\right) T_{A}$ has a projective cover in add $T_{A}$.

$\left(T_{4}\right)$ If $M_{A}$ is a module with $\operatorname{Hom}_{A}\left(T_{A}, M_{A}\right)=0=\operatorname{Ext}_{A}^{1}\left(T_{A}, M_{A}\right)$, then $M_{A}=0$.

Assume $T_{A}$ satisfies $\left(T_{1}\right),\left(T_{2}\right),\left(T_{3}\right),\left(T_{4}\right)$, and let $B=\operatorname{End}\left(T_{A}\right)$. Let $\pi: P_{A} \rightarrow T_{A}$ be a projective cover of $T_{A}$, with kernel $\mu: K_{A} \rightarrow P_{A}$. Then $P_{A} \in$ add $T_{A}$, according to $\left(T_{3}\right)$, and $K_{A}$ is projective, according to $\left(T_{1}\right)$. It has been shown in Proposition 5 of [6] that any indecomposable projective module is a direct summand of $P_{A} \oplus K_{A}$. Hence the result follows from Remark 1 in (2.1).

3. Isometry of Grothendieck groups. We fix a tilting module $T_{A}$, and let $B=$ $\operatorname{End}\left(T_{A}\right)$.

(3.1) Recall that $G_{0}(A)$ denotes the Grothendieck group of $A$, it is a free abelian group with basis the set $\{R(a) \mid a\}$ of simple right $A$-modules, and therefore can be identified with some $\mathbf{Z}^{m}$. For any right $A$-module $M_{A}$, the dimension vector $\operatorname{dim} M$ is the element of $G_{0}(A)=\mathbf{Z}^{m}$ with components

$$
(\operatorname{dim} M)_{a}=\left|\operatorname{Hom}_{A}(P(a), M)_{\text {End } P(a)}\right|
$$

(where $P(a)$ is projective with $P(a) / \operatorname{rad} P(a)=R(a)$ ), this is just the number of composition factors of $M_{A}$ of the form $R(a)$ in any fixed composition series.

LEMMA. If $X_{A}$ is a module with p.d. $X_{A} \leqslant 1$, then

$$
d_{X}\left(M_{A}\right)=\left|\operatorname{Hom}_{A}(X, M)_{\operatorname{End}(X)}\right|-\left|\operatorname{Ext}_{A}^{1}(X, M)_{\operatorname{End}(X)}\right|
$$

defines a linear mapping $G_{0}(A) \rightarrow \mathbf{Z}$.

Proof. Given an exact sequence

$$
0 \rightarrow M^{\prime} \rightarrow M \rightarrow M^{\prime \prime} \rightarrow 0
$$

the long exact sequence obtained by applying $\operatorname{Hom}_{A}\left({ }_{A} X_{\operatorname{End}(X)},-\right)$ is a sequence of End $(X)$-modules, and immediately shows the additivity formula

$$
d_{X}(M)=d_{X}\left(M^{\prime}\right)+d_{X}\left(M^{\prime \prime}\right) .
$$

This finishes the proof.

(3.2) Proposition. Let $T_{A}$ be a tilting module, with $B=\operatorname{End}\left(T_{A}\right)$. Then there exists a group isomorphism $f: G_{0}(A) \rightarrow G_{0}(B)$ satisfying

$$
f\left(\operatorname{dim} M_{A}\right)=\operatorname{dim} F\left(M_{A}\right)-\operatorname{dim} F^{\prime}\left(M_{A}\right) .
$$

Also, $f$ can be calculated as follows: Let $T(1), \ldots, T(n)$ be a full set of representatives of the indecomposable direct summands of $T_{A}$, let $Q(i)_{B}=F(T(i))$, and $S(i)_{B}=$ $Q(i) / \operatorname{rad} Q(i)$. Then

$$
f\left(\operatorname{dim} M_{A}\right)=\sum_{i=1}^{n} d_{T(i)}\left(M_{A}\right) \cdot S(i) .
$$


Proof. Define

$$
f\left(M_{A}\right)=\operatorname{dim} F\left(M_{A}\right)-\operatorname{dim} F^{\prime}\left(M_{A}\right) .
$$

Now given an exact sequence

$$
0 \rightarrow M_{A}^{\prime} \rightarrow M_{A} \rightarrow M_{A}^{\prime \prime} \rightarrow 0
$$

of $A$-modules, we obtain the exact sequence of $B$-modules

$$
0 \rightarrow F\left(M^{\prime}\right) \rightarrow F(M) \rightarrow F\left(M^{\prime \prime}\right) \rightarrow F^{\prime}\left(M^{\prime}\right) \rightarrow F^{\prime}(M) \rightarrow F^{\prime}\left(M^{\prime \prime}\right) \rightarrow 0 ;
$$

thus, in $G_{0}(B)$, we have the equality

$$
f(M)=f\left(M^{\prime}\right)+f\left(M^{\prime \prime}\right),
$$

and therefore $f$ induces a linear map $G_{0}(A) \rightarrow G_{0}(B)$, again denoted by $f$.

On the other hand, by the previous lemma, we also have a linear mapping $G_{0}(A) \rightarrow G_{0}(B)$ induced by

$$
\tilde{f}\left(M_{A}\right)=\sum_{i=1}^{n} d_{T(i)}\left(M_{A}\right) \cdot S(i) .
$$

In order to show that $\tilde{f}=f$, consider first a module $M_{A} \in \mathcal{T}$; thus

$$
f\left(M_{A}\right)=\operatorname{dim} F\left(M_{A}\right) \text {. }
$$

The $i$ th component of this element of $G_{0}(B)=\mathbf{Z}^{n}$ is given by

$$
\begin{aligned}
f\left(M_{A}\right)_{i} & =\left(\operatorname{dim} F\left(M_{A}\right)\right)_{i}=\left|\operatorname{Hom}_{A}\left(Q(i), F\left(M_{A}\right)\right)_{\text {End } Q(i)}\right| \\
& =\left|\operatorname{Hom}_{A}\left(F(T(i)), F\left(M_{A}\right)\right)_{\text {End } F T(i)}\right| \\
& =\operatorname{Hom}_{A}\left(T(i), M_{A}\right)_{\text {End } T(i)} \mid=d_{T(i)}\left(M_{A}\right)=\tilde{f}\left(M_{A}\right)_{i}
\end{aligned}
$$

using the fact that $Q(i)=F(T(i))$, and that $F$ induces a categorial equivalence between $\mathcal{T}$ and $\mathcal{Y}$. On the other hand, consider also $M_{A} \in \mathcal{F}$; thus

$$
f\left(M_{A}\right)=-\operatorname{dim} F^{\prime}\left(M_{A}\right) \text {. }
$$

We denote by $J(i)$ the injective $B$-module with $\operatorname{soc} J(i)=S(i)$. We have $G^{\prime} J(i)=$ $\tau T(i)$, according to the connecting lemma. So since both $M_{A}$ and $\tau T(i)$ belong to $\mathscr{F}$, and p.d. $T(i) \leqslant 1$, we have

$$
\begin{aligned}
\operatorname{Hom}_{B}\left(F^{\prime}(M), J(i)\right) & =\operatorname{Hom}_{B}\left(F^{\prime}(M), F^{\prime} G^{\prime} J(i)\right) \\
& =\operatorname{Hom}_{B}\left(F^{\prime}(M), F^{\prime} \tau T(i)\right) \\
& \approx \operatorname{Hom}_{A}(M, \tau T(i)) \approx D \operatorname{Ext}^{1}(T(i), M)
\end{aligned}
$$

As a consequence, we see that

$$
\begin{aligned}
f\left(M_{A}\right)_{i} & =\left(\operatorname{dim} F^{\prime}\left(M_{A}\right)\right)_{i}=-\operatorname{End}(J(i)) \\
& =-\frac{\operatorname{dim}_{k} \operatorname{Hom}\left(F^{\prime}(M), J(i)\right)}{\operatorname{dim}_{k} \operatorname{End} J(i)} \\
& =-\frac{\operatorname{dim}_{\operatorname{Ext}^{1}(T(i), M)}}{\operatorname{dim}_{k} \operatorname{End} T(i)}=\tilde{f}\left(M_{A}\right)_{i} .
\end{aligned}
$$


(We also have used that End $J(i) \approx$ End $Q(i) \approx$ End $T(i)$.) Since any simple $A$-module belongs to $\mathscr{F}$ or to $\mathcal{T}$, we see that $f$ and $\tilde{f}$ coincide on a generating set of $G_{0}(A)$; thus $f=\tilde{f}$.

It remains to be seen that $f$ is bijective. Any simple $B$-module lies in $\mathfrak{X}$ or in $\mathscr{Y}$, since $(X, \mathscr{Y})$ is a torsion theory; thus it is either the image under $F$ of a module from $\mathcal{T}$, or the image under $F^{\prime}$ of a module from $\mathcal{F}$. It follows that the image of $f$ contains the canonical generating set of $G_{0}(B)$. Thus $f$ is surjective, and consequently rank $G_{0}(A) \geqslant \operatorname{rank} G_{0}(B)$. By symmetry, also rank $G_{0}(B) \geqslant \operatorname{rank} G_{0}(A)$; thus $G_{0}(A)$ and $G_{0}(B)$ have the same rank. This implies that the surjective map $f$ is in fact bijective.

COROLlaRy. If $T_{A}$ is a tilting module, with $B=\operatorname{End}\left(T_{A}\right)$, then the number of simple $A$-modules coincides with the number of simple B-modules.

(3.2) Proposition. The Euler characteristic is defined for $A$ if and only if it is defined for B. In this case, $f$ is an isometry.

Proof. Assume, $P(1), \ldots, P(n)$ are the indecomposable projective $A$-modules, and $\operatorname{dim} P(a), 1 \leqslant a \leqslant n$, is a basis for $G_{0}(A) \otimes \mathbf{Q}$. For any $P(a)$, we have an exact sequence

$$
0 \rightarrow P(a) \rightarrow T^{\prime} \rightarrow T^{\prime \prime} \rightarrow 0
$$

with $T^{\prime}, T^{\prime \prime} \in \operatorname{add}(T)$; thus we see that $G_{0}(A) \otimes \mathbf{Q}$ also is generated by the elements $\operatorname{dim} T(i), 1 \leqslant i \leqslant n$, where the $T(i)$ are pairwise nonisomorphic direct summands of $T_{A}$. Under $f$, the vectors $\operatorname{dim} T(i)$, are mapped to $\operatorname{dim} F T(i)$; thus $\operatorname{dim} F T(i), 1 \leqslant i \leqslant$ $n$, is a basis of $G_{0}(B) \otimes \mathbf{Q}$. However, the modules $F T(i), 1 \leqslant i \leqslant n$, are the indecomposable projective $B$-modules. This shows that also the Euler characteristic of $B$ is defined.

In order to show that $f$ is an isometry, we only have to consider a basis in $G_{0}(A) \otimes \mathbf{Q}$. We will use the basis $\operatorname{dim} T(i), 1 \leqslant i \leqslant n$. Note that

$$
\langle\operatorname{dim} F T(i), \operatorname{dim} F T(j)\rangle=\operatorname{dim}_{k} \operatorname{Hom}(F T(i), F T(j)),
$$

since $F T(i)$ is projective. Also

$$
\langle\operatorname{dim} T(i), \operatorname{dim} T(j)\rangle=\operatorname{dim}_{k} \operatorname{Hom}(T(i), T(j)),
$$

since p.d. $T(i) \leqslant 1$, and $\operatorname{Ext}^{1}(T(i), T(j))=0$. Thus

$$
\begin{aligned}
\langle f \operatorname{dim} T(i), f \operatorname{dim} T(j)\rangle & =\langle\operatorname{dim} F T(i), \operatorname{dim} F T(j)\rangle \\
& =\operatorname{dim}_{k} \operatorname{Hom}(F T(i), F T(j)) \\
& =\operatorname{dim}_{k} \operatorname{Hom}(T(i), T(j))=\langle\operatorname{dim} T(i), \operatorname{dim} T(j)\rangle .
\end{aligned}
$$

\section{Tilting modules for hereditary algebras.}

(4.1) Lemma. Let $A$ be hereditary. If $T_{1}, T_{2}$ are indecomposable with $\operatorname{Ext}^{1}\left(T_{1}, T_{2}\right)=0$, then any nonzero map $T_{2} \rightarrow T_{1}$ is an epimorphism or a monomorphism. In particular, if $T_{1}$ is indecomposable with $\operatorname{Ext}^{1}\left(T_{1}, T_{1}\right)=0$, then $\operatorname{End}\left(T_{1}\right)$ is a division ring. 
Proof. Let $\varphi: T_{2} \rightarrow T_{1}$ be nonzero, and assume $\varphi$ is neither an epimorphism nor a monomorphism. Let $U$ be the image of $\varphi$, and $\varphi=\mu \varepsilon$, where $\varepsilon: T_{2} \rightarrow U$, is surjective, and $\mu: U \hookrightarrow T_{1}$ is the inclusion. With $\varepsilon$ also $\operatorname{Ext}^{1}\left(T_{1} / U, \varepsilon\right)$ is surjective; thus we obtain a module $V$ and a commutative diagram:

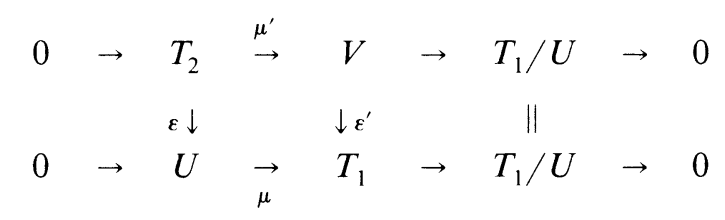

However, this implies that the sequence

$$
0 \rightarrow T_{2} \stackrel{\left(-\varepsilon^{\prime}\right)}{\rightarrow} U \oplus \stackrel{\left(\mu \varepsilon^{\prime}\right)}{\rightarrow} T_{1} \rightarrow 0
$$

is exact. Note that this sequence cannot split. (Otherwise $T_{1} \oplus T_{2} \approx U \oplus V$, and by Krull-Schmidt $U$ would have to be isomorphic to one of $T_{1}, T_{2}$. $)$ Thus $\operatorname{Ext}^{1}\left(T_{1}, T_{2}\right) \neq$ 0 .

(4.2) Corollary. Let $A$ be hereditary, and $T_{A}$ a module satisfying $\operatorname{Ext}_{A}^{1}\left(T_{A}, T_{A}\right)=0$. Then the quiver of $B=\operatorname{End}\left(T_{A}\right)$ has no oriented cycles.

Proof. Let $T_{i}, T_{j}, T_{k}$ be indecomposable modules in $\operatorname{add}\left(T_{A}\right)$, and $\varphi: T_{i} \rightarrow T_{j}, \quad \psi$ : $T_{j} \rightarrow T_{k}$ nonzero maps. Either both maps are monos, or both are epis, or $\varphi$ is mono, $\psi$ is epi. Otherwise $\varphi$ is a proper epimorphism and $\psi$ a proper monomorphism, according to 4.1 , and therefore $\psi \varphi$ is nonzero and neither mono nor epi, contrary to 4.1.

Thus, assume there is a sequence of nonzero maps

$$
T_{1} \rightarrow T_{2} \rightarrow \cdots \rightarrow T_{n} \rightarrow T_{1}
$$

with all $T_{i}$ indecomposable and in $\operatorname{add}\left(T_{A}\right)$. By the previous consideration, either all maps are mono, or all maps are epi (since otherwise we obtain an epi followed by a mono), thus all maps have to be isomorphisms.

(4.3) Lemma. Let $A$ be hereditary. Let $T(1), \ldots, T(s)$ be pairwise nonisomorphic $A$-modules, and $T_{A}=\bigoplus_{i=1}^{s} T(i)$. If $\operatorname{Ext}_{A}^{1}(T, T)=0$, then the vectors $\operatorname{dim} T(i)$ in $G_{0}(A)$ are linearly independent. In particular, $s \leqslant \operatorname{rank} G_{0}(A)$.

In the case of an algebraically closed base field, this result was first established by Happel in [9].

Proof. We can assume that the $T(i)$ are ordered in such a way that $\operatorname{Hom}_{A}(T(i), T(j))=0$ for $i>j$, due to 4.2 , and note that $\operatorname{End}_{A}(T(i))$ is a division ring for all $i$. In 3.1 , we have introduced linear mappings $d_{i}=d_{T(i)}: G_{0}(A) \rightarrow \mathbf{Z}$; thus we obtain a map

$$
d=\left(d_{1}, \ldots, d_{s}\right): G_{0}(A) \rightarrow \mathbf{Z}^{s} .
$$

The images of $\operatorname{dim} T(1), \ldots, \operatorname{dim} T(s)$ form a linearly independent generating set of $\mathbf{Z}^{s}$, since the matrix $\left(d_{i}(T(j))\right)_{i j}$ is a triangular matrix with all diagonal elements equal to 1 (for, $\operatorname{Ext}_{A}^{1}(T(i), T(j))=0$ for all $i, j$, thus $d_{i}(T(j))$ is just the dimension of $\mathrm{Hom}_{A}(T(i), T(j))$ as an $\operatorname{End}_{A}(T(i))$-vector space.) Therefore, $\operatorname{dim} T(1), \ldots, \operatorname{dim} T(s)$ themselves have to be linearly independent. 
(4.4) Lemma. Let $A$ be hereditary, with $n$ simple modules. Let $T(1), \ldots, T(n)$ be pairwise nonisomorphic A-modules, and $T_{A}=\bigoplus_{i=1}^{n} T(i)$. Suppose $\operatorname{Ext}_{A}^{1}(T, T)=0$. Then, for any $A$-module $M_{A}$, there exists an exact sequence of the form

$$
0 \rightarrow T_{A}^{\prime} \rightarrow M_{A}^{\prime} \rightarrow M_{A} \rightarrow 0,
$$

with $T^{\prime} \in$ add $T_{A}$, and $M_{A}^{\prime}$ cogenerated by $T_{A}$.

Proof. We may assume $M_{A}$ indecomposable. First we show that $\operatorname{Ext}_{A}^{1}(M, T)=0$ implies $\operatorname{Hom}_{A}(M, T) \neq 0$. Consider the additive map $d_{M}: G_{0}(A) \rightarrow \mathbf{Z}$ given in 3.1. For any injective module $I$ with $\operatorname{Hom}_{A}(M, I) \neq 0$, we have $d_{M}(I) \neq 0$, thus $d_{M} \neq 0$. Now the elements $\operatorname{dim} T(1), \ldots, \operatorname{dim} T(n)$ are linearly independent in $G_{0}(A)$, according to 4.3; thus, since $G_{0}(A)$ has rank $n$, they form a basis of $G_{0}(A) \otimes \mathbf{Q}$; thus $d_{M}(\operatorname{dim} T(i)) \neq 0$ for some $i$. Since $\operatorname{Ext}_{A}^{1}(M, T(i))=0$, we have $\operatorname{Hom}_{A}(M, T(i)) \neq 0$.

In fact, $\operatorname{Ext}^{1}(M, T)=0$ even implies that $M$ is cogenerated by $T$. Namely, let $M^{\prime}$ be the intersection of all kernels of maps $M \rightarrow T$. Then there is an embedding $M / M^{\prime} \hookrightarrow \oplus T$, and this inclusion shows that $\operatorname{Ext}^{1}\left(M / M^{\prime}, T\right)$ is an epimorphic image of $\operatorname{Ext}^{1}(\oplus T, T)$, and therefore zero. Thus the exact sequence $0 \rightarrow M^{\prime} \rightarrow M \rightarrow M / M^{\prime}$ $\rightarrow 0$ gives rise to an exact sequence

$$
0 \rightarrow \operatorname{Hom}\left(M / M^{\prime}, T\right) \rightarrow \operatorname{Hom}(M, T) \rightarrow \operatorname{Hom}\left(M^{\prime}, T\right) \rightarrow 0,
$$

but, since every map $M \rightarrow T$ factors over $M / M^{\prime}$, we see that $\operatorname{Hom}\left(M^{\prime}, T\right)=0$. On the other hand, the inclusion $M^{\prime} \hookrightarrow M$ also gives rise to an epimorphism $\operatorname{Ext}^{1}(M, T) \rightarrow \operatorname{Ext}^{1}\left(M^{\prime}, T\right)$; thus it follows that also $\operatorname{Ext}^{1}\left(M^{\prime}, T\right)=0$. By the first part of the proof, we can conclude $M^{\prime}=0$, thus $M$ is cogenerated by $T$.

Now, let $E_{1}, \ldots, E_{m}$ be a basis of $\operatorname{Ext}^{1}(M, T)$ and let

$$
\left(E_{i}\right)_{i}: 0 \rightarrow \bigoplus_{i=1}^{m} T \rightarrow M^{\prime} \rightarrow M \rightarrow 0
$$

be the corresponding exact sequence. Let $T^{\prime}=\bigoplus_{i=1}^{m} T$. Applying $\operatorname{Hom}(-, T)$, we obtain an exact sequence

$$
\operatorname{Hom}\left(T^{\prime}, T\right) \rightarrow \operatorname{Ext}^{1}(M, T) \rightarrow \operatorname{Ext}^{1}\left(M^{\prime}, T\right) \rightarrow 0,
$$

where we use $\operatorname{Ext}^{1}\left(T^{\prime}, T\right)=0$. However, the canonical projections in $\operatorname{Hom}\left(\oplus_{i=1}^{m} T, T\right)$ obviously go to the elements $E_{1}, \ldots, E_{m}$, thus the first map is surjective, and therefore $\operatorname{Ext}^{1}\left(M^{\prime}, T\right)=0$. By the previous part of the proof, this shows that $M^{\prime}$ is cogenerated by $T$.

(4.5) Theorem. Let $A$ be hereditary, and $T_{A}$ an $A$-module. Then $T_{A}$ is a tilting module if and only if $\operatorname{Ext}_{A}^{1}\left(T_{A}, T_{A}\right)=0$ and the number of isomorphism classes of indecomposable direct summands of $T_{A}$ is equal to the number of isomorphism classes of simple A-modules.

Proof. We can assume $T_{A}=\bigoplus_{i=1}^{s} T(i)$, with pairwise nonisomorphic indecomposable modules $T(i)$. Let $n$ be the number of simple $A$-modules. If $T_{A}$ is a tilting module, then $s=n$, according to Corollary 3.2.

Conversely, let $\operatorname{Ext}_{A}^{1}(T, T)=0$, and $s=n$. We apply Lemma 4.4 to $M=P(j)$, and obtain an exact sequence

$$
0 \rightarrow T_{A}^{\prime} \rightarrow M_{A}^{\prime} \rightarrow P(j) \rightarrow 0,
$$


with $T_{A}^{\prime} \in$ add $T$, and $M_{A}^{\prime}$ cogenerated by $T_{A}$. The sequence splits, thus $P(j)$ itself is cogenerated by $T_{A}$. Let $f_{1}, \ldots, f_{r}$ be a basis of $\operatorname{Hom}_{A}(P(j), T)$, then $f=\left(f_{i}\right)_{i}$ : $P(j) \rightarrow \oplus T$ is mono. Let $V$ be the cokernel; thus there is an exact sequence

$$
0 \rightarrow P(j) \stackrel{f}{\rightarrow} \bigoplus T \rightarrow V \rightarrow 0 .
$$

Since $\oplus T$ maps onto $V$, also $\operatorname{Ext}^{1}(T, \oplus T)$ maps onto $\operatorname{Ext}^{1}(T, V)$; thus $\operatorname{Ext}^{1}(T, V)$ $=0$. Applying $\operatorname{Hom}_{A}(-, T)$ to the given exact sequence, we get an exact sequence

$$
\operatorname{Hom}(\oplus T, T) \rightarrow \operatorname{Hom}(P(j), T) \rightarrow \operatorname{Ext}^{1}(V, T) \rightarrow 0,
$$

since $\operatorname{Ext}^{1}(T, T)=0$. The first map is surjective by construction of $f$; thus $\operatorname{Ext}^{1}(V, T)=0$. Finally, the epimorphism $\oplus T \rightarrow V$ gives rise to an epimorphism $0=\operatorname{Ext}^{1}(V, \oplus T) \rightarrow \operatorname{Ext}^{1}(V, V)$; thus also $\operatorname{Ext}^{1}(V, V)=0$. We conclude that

$$
\operatorname{Ext}_{A}^{1}(T \oplus V, T \oplus V)=0 \text {. }
$$

It follows from Lemma 4.3 that $V$ belongs to add $T$, since otherwise we get too many linearly independent vectors in $G_{0}(A)$. This shows that $T_{A}$ also satisfies the last condition of a tilting module, and finishes the proof.

Corollary. Let $A$ be hereditary and $T_{A}$ a tilting module. Then $D\left(T_{A}\right)$ also is a tilting module.

REMARK. Let $T_{A}$ be a tilting module with hereditary endomorphism ring $B$. Let $T_{A}$ be an injective cogenerator. Then the $B$-module $F\left(I_{A}\right)=\operatorname{Hom}\left({ }_{B} T_{A}, I_{A}\right)$ is also a tilting module, and End $F\left(I_{A}\right)=B$.

Proof. We can assume $I_{A}=D\left({ }_{A} A\right)$. Then

$$
F\left(I_{A}\right)=\operatorname{Hom}_{A}\left({ }_{B} T_{A}, I_{A}\right) \approx D \operatorname{Hom}\left(A_{A},{ }_{B} T_{A}\right) \approx D\left({ }_{B} T\right),
$$

and with ${ }_{B} T$ also $D\left({ }_{B} T\right)$ is a tilting module.

5. The global dimension of a tilted algebra. In this section, we will assume that $A$ is hereditary, $T_{A}$ a tilting module, and $B=\operatorname{End}\left(T_{A}\right)$; thus $B$ is a tilted algebra. Also, as before, let $\mathcal{X}$ be the set of $B$-modules of the form $\operatorname{Ext}^{1}\left({ }_{B} T_{A}, M_{A}\right)$, and $\mathcal{Y}$ the set of $B$-modules of the form $\operatorname{Hom}\left({ }_{B} T_{A}, M_{A}\right)$.

(5.1) LemMa. p.d. $N_{B} \leqslant 1$ for all $N_{B} \in \mathcal{Y}$.

Proof. Let $N_{B}=\operatorname{Hom}\left({ }_{B} T_{A}, M_{A}\right)$ where we can assume that $M_{A}$ belongs to $\widetilde{T}\left(T_{A}\right)$. Let $f_{1}, \ldots, f_{r}$ be a basis of $\operatorname{Hom}\left({ }_{B} T_{A}, M_{A}\right)$ and $f=\left(f_{i}\right)_{i}: \oplus T \rightarrow M$. Since $M \in \mathcal{T}\left(T_{A}\right)$, the map $f$ is surjective. Denote by $K$ its kernel. We have $\operatorname{Ext}_{A}^{1}(K, T)=0$, since $K$ embeds into $\oplus T$, and $\operatorname{Ext}_{A}^{1}(T, K)=0$ since $\operatorname{Hom}(T, f)$ is surjective. It follows that also $\operatorname{Ext}_{A}^{1}(K, K)=0$, again using that $K$ embeds into $\oplus T$. As a consequence

$$
\operatorname{Ext}_{A}^{1}(T \oplus K, T \oplus K)=0
$$

and therefore $K \in$ add (T), due to 4.3. Thus, the induced exact sequence

$$
0 \rightarrow \operatorname{Hom}\left({ }_{B} T_{A}, K_{A}\right) \rightarrow \operatorname{Hom}\left({ }_{B} T_{A}, \oplus T_{A}\right) \stackrel{\operatorname{Hom}(T, f)}{\rightarrow} \operatorname{Hom}\left({ }_{B} T_{A}, M_{A}\right) \rightarrow 0
$$

is a projective resolution of $N_{B}=\operatorname{Hom}\left({ }_{B} T_{A}, M_{A}\right)$.

(5.2) THEOREM. gl. dim. $B \leqslant 2$ for any tilted algebra. 
Proof. If $N_{B} \in \mathcal{Y}$, then p.d. $N_{B} \leqslant 1$, by the previous lemma. Thus, consider now the case $N_{B} \in \mathcal{X}$, say $N_{B}=\operatorname{Ext}^{1}\left({ }_{B} T_{A}, M_{A}\right)$ for some $M_{A} \in \mathcal{F}\left(T_{A}\right)$. By 4.4 , we find an exact sequence

$$
0 \rightarrow \oplus T_{A} \stackrel{f}{\rightarrow} M_{A}^{\prime} \stackrel{p}{\rightarrow} M_{A} \rightarrow 0
$$

with $M_{A}^{\prime}$ cogenerated by $T_{A}$. Let $g_{1}, \ldots, g_{r}$ be a basis of $\operatorname{Hom}_{A}\left(M_{A}^{\prime}, T_{A}\right)$, and $g=\left(g_{i}\right): M^{\prime} \rightarrow T^{\prime}=\bigoplus_{i=1}^{r} T_{A}$. Note that $g$ is an inclusion, say with cokernel $V$. We claim that $\operatorname{Ext}^{1}(T \oplus V, T \oplus V)=0$. By the universality of $g$, we have $\operatorname{Ext}^{1}(V, T)=0$. Since there is a surjection $\oplus T \rightarrow V$, and $\operatorname{Ext}^{1}(T, \oplus T)=0, \operatorname{Ext}^{1}(V, \oplus T)=0$, we conclude that both $\operatorname{Ext}^{1}(T, V)=0=\operatorname{Ext}^{1}(V, V)$. From $\operatorname{Ext}^{1}(T \oplus V, T \oplus V)=0$, it follows by 4.3 that $V \in$ add $T$. Applying $\operatorname{Hom}_{A}\left({ }_{B} T_{A},-\right)$ to the sequence

$$
0 \rightarrow M^{\prime} \stackrel{g}{\rightarrow} T^{\prime} \rightarrow V \rightarrow 0
$$

we obtain the following exact sequence of $B$-modules:

$(*) \quad 0 \rightarrow \operatorname{Hom}_{A}\left(T, M^{\prime}\right) \rightarrow \operatorname{Hom}_{A}\left(T, T^{\prime}\right) \rightarrow \operatorname{Hom}_{A}(T, V) \rightarrow \operatorname{Ext}^{1}\left(T, M^{\prime}\right) \rightarrow 0$.

Since $T^{\prime}, V \in$ add $T_{A}$, the $B$-modules $\operatorname{Hom}_{A}\left(T, T^{\prime}\right)$ and $\operatorname{Hom}_{A}(T, V)$ are projective. Since $\operatorname{Hom}_{A}(T, M)=0$, any homomorphism $T \rightarrow M^{\prime}$ maps into the image of $f$; thus

$$
\operatorname{Hom}(T, f): \operatorname{Hom}(T, \oplus T) \rightarrow \operatorname{Hom}\left(T, M^{\prime}\right)
$$

is an isomorphism of $B$-modules, and therefore also $\operatorname{Hom}_{A}\left({ }_{B} T_{A}, M_{A}^{\prime}\right)$ is projective. Finally, the map $p$ induces an isomorphism

$$
\operatorname{Ext}_{A}^{1}(T, p): \operatorname{Ext}^{1}\left(T, M^{\prime}\right) \rightarrow \operatorname{Ext}^{1}(T, M)
$$

thus we see that $(*)$ is a projective resolution of $N_{B}=\operatorname{Ext}^{1}\left({ }_{B} T_{A}, M_{A}\right)$, and therefore p.d. $N_{B} \leqslant 2$. Since any $B$-module is an extension of a module from $\mathfrak{X}$ by a module from $\mathcal{Y}$, we conclude gl. $\operatorname{dim} B \leqslant 2$.

(5.3) As we have seen, the indecomposable modules from $\mathcal{Y}$ all have projective dimension 0 or 1 , those of $\mathfrak{X}$ all have projective dimension 1 or 2 (here we use the fact that all projective modules belong to $\mathscr{Y})$. For certain modules in $\mathfrak{X}$ one can decide which value actually occurs.

Lemma. Assume that all simple projective modules belong to add $T_{A}$. Let $P_{A}$ be indecomposable projective, and not in add $T_{A}$. Then p.d. $F^{\prime}\left(P_{A}\right)=2$.

Proof. There is an exact sequence

$$
0 \rightarrow P_{A} \stackrel{f}{\rightarrow} T_{A}^{\prime} \rightarrow T_{A}^{\prime \prime} \rightarrow 0
$$

with $T^{\prime}, T^{\prime \prime} \in$ add $T_{A}$. We apply $\operatorname{Hom}_{A}\left({ }_{B} T_{A},-\right)$ and obtain the following exact sequence of $B$-modules:

$$
0 \rightarrow F\left(P_{A}\right) \stackrel{F(f)}{\rightarrow} F\left(T_{A}^{\prime}\right) \rightarrow F\left(T_{A}^{\prime \prime}\right) \rightarrow F^{\prime}\left(P_{A}\right) \rightarrow 0 .
$$

Let $U_{A}$ be the torsion submodule of $P_{A}$, with inclusion map $u$. Then

$$
F(u)=\operatorname{Hom}_{A}\left({ }_{B} T_{A}, u\right): \operatorname{Hom}_{A}\left({ }_{B} T_{A}, U_{A}\right) \rightarrow \operatorname{Hom}_{A}\left({ }_{B} T_{A}, P_{A}\right)
$$


clearly is an isomorphism of $B$-modules. Now $U_{A}$ is projective (being a submodule of $P_{A}$ ), on the other hand, $U_{A}$ is generated by $T_{A}$ (being a torsion module); thus $U_{A} \in$ add $T_{A}$, and therefore $F\left(P_{A}\right) \approx \operatorname{Hom}_{A}\left({ }_{B} T_{A}, U_{A}\right)$ is a projective $B$-module. Thus, (*) is a projective resolution of $F^{\prime}\left(P_{A}\right)$. Assume the projective dimension of $F^{\prime}\left(P_{A}\right)$ is $\leqslant 1$. Then $F(f)$ is split mono, and therefore also $F(f u)$. Now $f u: U_{A} \rightarrow T_{A}^{\prime}$ is a map inside $\mathcal{T}\left(T_{A}\right)$; thus $f u$ is isomorphic to $G F(f u)$. Since with $F(f u)$ also $f u \approx G F(f u)$ is split mono, we finally conclude that $u: U_{A} \rightarrow P_{A}$ is split mono. However, $u$ being a proper monomorphism (since $P_{A} \notin$ add $T_{A}$, and $U_{A} \in$ add $T_{A}$ ), and $P_{A}$ indecomposable, we conclude that $U_{A}=0$. However, this is a contradiction to the fact that $U_{A} \supseteq \operatorname{soc} P_{A}$, since, by assumption, all simple projective modules are torsion modules.

6. Splitting of the torsion theory ( $\mathfrak{X}, \mathcal{Y})$. We assume throughout that $A$ is hereditary, $T_{A}$ a tilting module, with endomorphism ring $B$. In $\mathfrak{N}_{A}$, we consider the torsion theory $(\mathcal{T}, \mathscr{F})$, in $\mathfrak{T}_{B}$ the torsion theory ( $\left.\mathscr{X}, \mathscr{Y}\right)$ derived from ${ }_{B} T_{A}$.

Always, $P(a)$ will denote an indecomposable projective module. The corresponding indecomposable injective module will be denoted by $I(a)$, it satisfies $\operatorname{soc} I(a)=$ $P(a) / \operatorname{rad} P(a)$.

(6.1) Proposition. The minimal left almost split map starting with FI(a) is of the form

$$
F I(a) \rightarrow F(I(a) / \operatorname{soc}) \oplus F^{\prime}(\operatorname{rad} P(a))
$$

Proof. (1) If $P(a) \notin$ add $T$, then there exists a module $E(a)$ and a nonsplit exact sequence

$$
0 \rightarrow F I(a) \rightarrow E(a) \rightarrow F^{\prime} P(a) \rightarrow 0
$$

and an exact sequence

$$
0 \rightarrow F^{\prime} \operatorname{rad} P(a) \rightarrow E(a) \rightarrow F(I(a) / \mathrm{soc}) \rightarrow 0 .
$$

Proof. We have to distinguish two cases. Let $S(a)=\operatorname{soc} I(a)$.

Case 1. $S(a)$ is a torsion module.

Since $F^{\prime} S(a)=0$, we obtain from the exact sequence

$$
0 \rightarrow S(a) \rightarrow I(a) \rightarrow I(a) / \mathrm{soc} \rightarrow 0
$$

an exact sequence

$$
0 \rightarrow F S(a) \rightarrow F I(a) \rightarrow F(I(a) / \mathrm{soc}) \rightarrow 0 .
$$

Since $P(a)$ is not a summand of $T$, any homomorphism $T \rightarrow P(a)$ maps into $\operatorname{rad} P(a)$; thus the induced map $F \operatorname{rad} P(a) \rightarrow F P(a)$ is an isomorphism. This, together with $F^{\prime} S(a)=0$ shows that we obtain from the exact sequence

$$
0 \rightarrow \operatorname{rad} P(a) \rightarrow P(a) \rightarrow S(a) \rightarrow 0
$$

an exact sequence

$$
0 \rightarrow F S(a) \rightarrow F^{\prime} \operatorname{rad} P(a) \rightarrow F^{\prime} P(a) \rightarrow 0 .
$$


Now form the pushout of these two sequences:

$$
\begin{aligned}
& \begin{array}{ll}
0 & 0 \\
\downarrow & \downarrow
\end{array} \\
& 0 \rightarrow F S(a) \quad \stackrel{\alpha}{\rightarrow} \quad F^{\prime} \operatorname{rad} P(a) \quad \rightarrow \quad F^{\prime} P(a) \quad \rightarrow \quad 0 \\
& \downarrow \beta \quad \downarrow \beta^{\prime} \quad \| \\
& 0 \rightarrow \quad F I(a) \quad \stackrel{\alpha^{\prime}}{\rightarrow} \quad E(a) \quad \stackrel{\pi}{\rightarrow} \quad F^{\prime} P(a) \quad \rightarrow \quad 0
\end{aligned}
$$

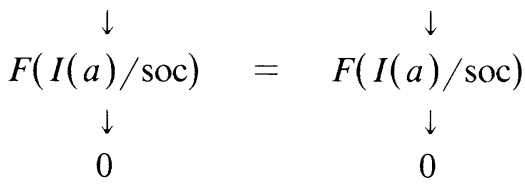

The short exact sequence starting with $F I(a)$ does not split. For, otherwise $\beta^{\prime}$ would be a map from the torsion module $F^{\prime} \operatorname{rad} P(a)$ into $E(a)=F I(a) \oplus F^{\prime} P(a)$, and therefore would map into $F^{\prime} P(a)$. As a consequence, $\pi \beta^{\prime} \alpha$ would be a monomorphism. However, $\pi \beta^{\prime} \alpha=\pi \alpha^{\prime} \beta=0$, and $F S(a) \neq 0$.

Case 2. $S(a)$ is torsionfree.

Since $F S(a)=0$, we obtain from $(* * *)$ the exact sequence

$$
0 \rightarrow F^{\prime} \operatorname{rad} P(a) \rightarrow F^{\prime} P(a) \rightarrow F^{\prime} S(a) \rightarrow 0 .
$$

Since $F S(a)=0, F^{\prime} I(a)=0$, we obtain from $(* *)$ the exact sequence

$$
0 \rightarrow F I(a) \rightarrow F(I(a) / \mathrm{soc}) \rightarrow F^{\prime} S(a) \rightarrow 0 .
$$

We form the pullback:

$$
\begin{aligned}
& \begin{array}{ll}
0 & 0 \\
\downarrow & \downarrow
\end{array} \\
& F^{\prime} \operatorname{rad} P(a)=F^{\prime} \operatorname{rad} P(a) \\
& 0 \rightarrow F I(a) \stackrel{\mu}{\rightarrow} \quad E(a) \quad \stackrel{\delta^{\prime}}{\rightarrow} \quad F^{\prime} P(a) \quad \rightarrow \quad 0 \\
& 0 \rightarrow F I(a) \rightarrow F(I(a) / \operatorname{soc}) \stackrel{\delta}{\rightarrow} \quad F^{\prime} S(a) \quad \rightarrow \quad 0 \\
& \downarrow
\end{aligned}
$$

Again, the short exact sequence ending with $F^{\prime} P(a)$ cannot split, since otherwise $\gamma^{\prime} \mu$, and therefore also $\delta \gamma^{\prime} \mu=\gamma \delta^{\prime} \mu=0$ would be surjective, contrary to $F^{\prime} S(a) \neq 0$.

(2) If $P(a) \notin$ add $T$, any nonsplit exact sequence

$$
0 \rightarrow F I(a) \rightarrow X \rightarrow F^{\prime} P(a) \rightarrow 0
$$

has to be an Auslander-Reiten sequence.

Proof. By $(2.3), \tau F^{\prime} P(a)=F I(a)$. Now End $F^{\prime} P(a) \approx$ End $P(a)$ is a division ring, thus $\operatorname{Ext}^{1}\left(F^{\prime} P(a), F I(a)\right)$ is one-dimensional as an End $F^{\prime} P(a)$-vector space.

(3) If $P(a) \in$ add $T$, then $F I(a)$ is indecomposable injective. Let $E(a)=$ $F(I(a) /$ soc $)$. Then again, there is an exact sequence of the form $(*)$. 
Proof. Since $P(a) \in$ add $T$, the cokernel of the canonical map $F \operatorname{rad} P(a) \rightarrow$ $F P(a)$ is simple, it is the simple module corresponding to the direct summand $P(a)$ in $B=\operatorname{End}\left(T_{A}\right)$, and we denote it by $U$. Thus $(* * *)$ gives rise to the exact sequence

$$
0 \rightarrow U \stackrel{\alpha}{\rightarrow} F S(a) \rightarrow F^{\prime} \operatorname{rad} P(a) \rightarrow 0,
$$

using the fact that $F^{\prime} P(a)=0$, since $P(a) \in$ add $T$. With $P(a)$ also $S(a)$ is a torsion module, then the sequence $(* *)$ gives the exact sequence

$$
0 \rightarrow F S(a) \stackrel{\beta}{\rightarrow} F I(a) \rightarrow F(I(a) / \text { soc }) \rightarrow 0 .
$$

Thus $F I(a)$ contains $F S(a)$ as a submodule, and this in turn the simple submodule $U$. Since $F I(a)$ is indecomposable injective, according to Corollary 2.3 , we see that $\beta \alpha U$ is the socle of $F I(a)$, and

$$
E(a)=F I(a) / \operatorname{soc}=F I(a) / \beta \alpha U
$$

is an extension of $F S(a) / \alpha U=F^{\prime} \operatorname{rad} P(a)$ by $F I(a) / \beta F S(a)=F(I(a) / \operatorname{soc})$.

(4) The sequence $(*)$ splits.

PROof. By induction along a source sequence. If $a$ is a source, then $\operatorname{rad} P(a)=0$, thus we have nothing to show. Consider now some $b$, and assume we know the splitting of all sequences for $a$, where $a \rightarrow b$ (recall that we write $a \rightarrow b$ in case $P(a) \in$ add $\operatorname{rad} P(b)$.) We want to show that $E(b)$ has a direct summand isomorphic to $F^{\prime}$ rad $P(b)$. Recall the definition of the valued graph associated to $A$. The numbers $d_{a b}, d_{a b}^{\prime} \in \mathbf{N}$ are defined by

$$
\operatorname{rad} P(b)=\bigoplus_{a} P(a)^{d_{a b}}, \quad I(a) / \operatorname{soc}=\bigoplus_{b} I(b)^{d_{a b}^{\prime}} .
$$

If $f_{a}=\operatorname{dim}_{k}$ End $P(a)=\operatorname{dim}_{k}$ End $I(a)$, then

$$
f_{a} d_{a b}=d_{a b}^{\prime} f_{b} \text {. }
$$

It follows that

$$
F^{\prime} \operatorname{rad} P(b)=\bigoplus_{a} F^{\prime} P(a)^{d_{a b}}
$$

thus we have to show that in case $F^{\prime} P(a) \neq 0$, the module $E(b)$ contains $F^{\prime} P(a)^{d_{a b}}$ as a direct summand. So assume $F^{\prime} P(a) \neq 0, d_{a b} \neq 0$. Then $a \rightarrow b$, thus, by induction

$$
E(a) \approx F^{\prime} \operatorname{rad} P(a) \oplus F(I(a) / \mathrm{soc}) .
$$

Also, $F I(a)$ is not injective, thus we have the Auslander-Reiten sequence

$$
0 \rightarrow F I(a) \rightarrow E(a) \rightarrow F^{\prime} P(a) \rightarrow 0,
$$

due to (1), (2) and the connecting lemma. Now

$$
F(I(a) / \mathrm{soc})=\bigoplus_{b} F I(b)^{d_{a b}^{\prime}} .
$$

Thus $E(a)$ contains $F I(b)^{d_{a b}^{\prime}}$ as direct summand. It follows that the space $\operatorname{Irr}\left(F I(b), F^{\prime} P(a)\right)$ considered as an End $F I(b)$-vector space, has dimension $d_{a b}^{\prime}$; thus

$$
\operatorname{dim}_{k} \operatorname{Irr}\left(F I(b), F^{\prime} P(a)\right)=d_{a b}^{\prime} f_{b}
$$


due to the fact that End $F I(b) \approx$ End $I(b)$. Since $f_{a} d_{a b}=d_{a b}^{\prime} f_{b}$, and $f_{a}=$ $\operatorname{dim}_{k}$ End $F^{\prime} P(a)$, we see that the dimension of $\operatorname{Irr}\left(F I(b), F^{\prime} P(a)\right)$ as $F^{\prime} P(a)$-vector space is $d_{a b}$, thus for the left almost split map $F I(b) \rightarrow E(b)$, the module $E(b)$ contains $F^{\prime} P(a)^{d_{a b}}$ as direct summand. This shows that $E(b)$ has a direct summand of the form $F^{\prime} \operatorname{rad} P(b)$. Since the torsion part of $E(b)$ is isomorphic to $F^{\prime} \operatorname{rad} P(b)$, it follows that the torsion part of $E(b)$ splits off, as we wanted to show.

This finishes the proof.

(6.2) Corollary. Either $P(a) \in$ add $T_{A}$ (and then $F I(a)$ is injective), or else there is an Auslander-Reiten sequence of the form

$$
0 \rightarrow F I(a) \rightarrow F(I(a) / \mathrm{soc}) \oplus F^{\prime} \operatorname{rad} P(a) \rightarrow F^{\prime} P(a) \rightarrow 0 .
$$

Proof. This is an immediate consequence of the connecting lemma and the previous result.

The Auslander-Reiten sequences given by 6.2 will be called the connecting sequences.

(6.3) Theorem. Let $A$ be hereditary, $T_{A}$ a tilting module, and $B=\operatorname{End}\left(T_{A}\right)$. Let $\mathcal{X}=\left\{N_{B} \mid \operatorname{Tor}_{1}^{B}\left(N_{B},{ }_{B} T\right)=0\right\}, \mathcal{Y}=\left\{N_{B} \mid N_{B} \otimes_{B} T=0\right\}$. Then any indecomposable right $B$-module belongs either to $\mathcal{X}$ or to $\mathcal{Y}$.

Proof. (1) If $F^{\prime} P(a) \rightarrow X_{B}$ is irreducible, then $X \in \mathcal{X}$.

Proof. We may suppose $X_{B}$ indecomposable. We use induction on $a$ along a source sequence. Note that $X$ cannot be projective, since $F^{\prime} P(a)$ is in $\mathcal{X}$, and projective modules belong to $\mathcal{Y}$. Thus $\tau X \neq 0$, and there is an irreducible map $\tau X \rightarrow F^{\prime} P(a)$. The connecting sequence ending with $F^{\prime} P(a)$ shows that $\tau X$ is either of the form $F I(b)$ with $I(b) \in \operatorname{add}\left(I(a) /\right.$ soc), or of the form $F^{\prime} P(c)$, with $P(c) \in$ add $\operatorname{rad} P(a)$. In the first case,

$$
X=\tau^{-1} \tau X=\tau^{-1} F I(b)=F^{\prime} P(b)
$$

is in $\mathcal{X}$. Note that in case $a$ is a source, then $\operatorname{rad} P(a)=0$, thus only the first case can happen. In general, if $\tau X=F^{\prime} P(c)$, consider the Auslander-Reiten sequence ending with $X$

$$
0 \rightarrow F^{\prime} P(c) \rightarrow X^{\prime} \rightarrow X \rightarrow 0 .
$$

Since $c$ is a predecessor of $a$, we know by induction that $X^{\prime}$ belongs to $\mathcal{X}$. Thus, since $\mathcal{X}$ is closed under factor modules, also $X$ is in $\mathcal{X}$.

(2) For any $a$, the module $\tau^{-1} F^{\prime} P(a)$ is in $\mathcal{X}$.

Proof. In case $\tau^{-1} F^{\prime} P(a) \neq 0$, consider an Auslander-Reiten sequence

$$
0 \rightarrow F^{\prime} P(a) \rightarrow X \rightarrow \tau^{-1} F^{\prime} P(a) \rightarrow 0 .
$$

By (1), the module $X$ belongs to $\mathcal{X}$. Note that $\mathcal{X}$ is closed under factor modules.

(3) For any $Y$ in $\mathcal{Y}$, we have $\operatorname{Ext}^{\mathrm{l}}\left(Y, F^{\prime} P(a)\right)=0$.

Proof. Hom ${ }_{B}\left(\tau^{-1} F^{\prime} P(a), Y\right)$ maps onto $D \operatorname{Ext}_{B}^{1}\left(Y, F^{\prime} P(a)\right)$, and $\tau^{-1} F^{\prime} P(a)$ is a torsion module, whereas $Y$ is torsionfree.

(4) For $X \in \mathcal{X}, Y \in \mathcal{Y}$, we have $\operatorname{Ext}^{1}(Y, X)=0$. 
Proof. Let $X=F^{\prime} M$ for some $A$-module $M_{A}$, and take a projective cover $P_{A} \rightarrow M_{A}$. Since p.d. $T_{A} \leqslant 1$, the induced map $F^{\prime} P \rightarrow F^{\prime} M$ is surjective. Since p.d. $Y_{B}$ $\leqslant 1$ according to 5.1 , also the induced map

$$
\operatorname{Ext}^{1}\left(Y, F^{\prime} P\right) \rightarrow \operatorname{Ext}^{1}\left(Y, F^{\prime} M\right)=\operatorname{Ext}^{1}(Y, X)
$$

is surjective. By (3), the first group is zero, thus $\operatorname{Ext}^{1}(Y, X)=0$.

This finishes the proof.

(6.4) Corollary. If $A$ is hereditary and of finite representation type, $T_{A}$ a tilting module, and $B=\operatorname{End}\left(T_{A}\right)$; then the number of indecomposable B-modules is less than equal to the number of indecomposable A-modules.

PROOF. This follows directly from (6.3) and the theorem of Brenner and Butler.

(6.5) We want to describe the Auslander-Reiten quiver of a tilted algebra as far as possible. We need to know the possible structure of all Auslander-Reiten sequences.

Theorem. Let $A$ be hereditary, $T_{A}$ a tilting module, $B=\operatorname{End}\left(T_{A}\right)$. Let

$$
0 \rightarrow N_{B}^{\prime} \rightarrow N_{B} \rightarrow N_{B}^{\prime \prime} \rightarrow 0
$$

be an Auslander-Reiten sequence in $\Upsilon_{B}$. Then either the modules $N^{\prime}, N, N^{\prime \prime}$ all belong to $\mathcal{X}$, or all belong to $\mathcal{Y}$, or this is a connecting sequence.

Proof. (1) Let $M_{A}$ be indecomposable in $\mathcal{T}_{A}$. If $M_{A} \notin$ add $T_{A}$, then there exists $M_{A}^{\prime}$ in $\widetilde{\sigma}_{A}$, with $\operatorname{Ext}_{A}^{1}\left(M, M^{\prime}\right) \neq 0$. If $M_{A}$ is not injective, then there exists $M_{A}^{\prime \prime}$ in $\widetilde{J}_{A}$ with $\operatorname{Ext}_{A}^{1}\left(M^{\prime \prime}, M\right) \neq 0$.

Proof. First, assume $M_{A} \notin$ add $T_{A}$. By 4.3, $\operatorname{Ext}_{A}^{1}(T \oplus M, T \oplus M) \neq 0$. Now $\operatorname{Ext}_{A}^{1}(T, T)=0$, and $\operatorname{Ext}_{A}^{1}(T, M)=0$ (since $\left.M_{A} \in \mathcal{T}\right)$. Thus $\operatorname{Ext}_{A}^{1}(M, T \oplus M) \neq 0$.

Similarly, if $M_{A}$ is not injective, then $\operatorname{Ext}_{A}^{1}(I \oplus M, I \oplus M) \neq 0$, where $I$ is an injective cogenerator. Since $I$ is injective,

$$
\operatorname{Ext}_{A}^{1}(I \oplus M, I)=0 ;
$$

thus $\operatorname{Ext}_{A}^{1}(I \oplus M, M) \neq 0$.

(2) Let

$$
0 \rightarrow M_{A}^{\prime} \rightarrow M_{A} \stackrel{\pi}{\rightarrow} M_{A}^{\prime \prime} \rightarrow 0
$$

be a relative Auslander-Reiten sequence of $\mathcal{T}$. Then the image under $F$ is an Auslander-Reiten sequence $\mathcal{O}$.

Proof. Let $N_{B}$ be indecomposable in $\Re_{B}$, and $\varphi: N_{B} \rightarrow F M^{\prime \prime}$ a nonzero map. Now $N_{B}$ is either torsion or torsionfree. Since $F M^{\prime \prime}$ is torsionfree, and $\varphi \neq 0$, we see that also $N$ is torsionfree, say $N=F(U)$ for some $A$-module $U_{A}$ in $\mathcal{T}$, and $\varphi=F(\alpha)$ for some $\alpha: U_{A} \rightarrow M_{A}^{\prime \prime}$. Either $\alpha$ is an isomorphism (and then $\varphi$ is an isomorphism) or else $\alpha=\pi \alpha^{\prime}$ for some $\alpha^{\prime}$, and then $\varphi=F(\pi) F\left(\alpha^{\prime}\right)$.

(3) Let $N_{B}$ be indecomposable and in $\mathcal{Y}$. Then $\tau N_{B} \in \mathcal{Y}$. Also, either $\tau^{-1} N_{B} \in \mathcal{O}$ or else $N_{B}=F I(a)$ and $\tau^{-1} N_{B}=F^{\prime} P(a)$, for some indecomposable projective module $P(a)$ not belonging to add $T_{A}$.

Proof. Let $N_{B}=F\left(M_{A}\right)$, with $M_{A}$ indecomposable in $\mathcal{T}$. If $M_{A} \in \operatorname{add} T_{A}$ then $N_{B}$ is projective, thus $\tau N_{B}=0 \in \mathcal{Y}$. If $M_{A} \notin \operatorname{add} T_{A}$, then there exists $M_{A}^{\prime}$ in $\mathcal{T}$ with 
$\operatorname{Ext}_{A}^{1}\left(M, M^{\prime}\right) \neq 0$. By the results of Auslander-Smalø, there exists a relative Auslander-Reiten sequence in $\mathcal{T}$, ending in $M$. According to (2), its image under $F$ is an Auslander-Reiten sequence ending in $F(M)=N_{B}$. Since all its terms belong to $\mathcal{Y}$, we see that $\tau N_{B} \in \mathcal{O}$.

Consider now $\tau^{-1} N_{B}$, and again let $N_{B}=F\left(M_{A}\right)$ with $M_{A} \in \mathcal{T}$. If $M_{A}=I(a)$, then $\tau^{-1} N_{B}=F^{\prime} P(a)$, according to the connecting lemma. Note that in case $P(a) \in$ add $T_{A}$, we have $\tau^{-1} N_{B}=0 \in \mathcal{Y}$. Assume now $M_{A}$ is not injective. According to (1), there exists $M_{A}^{\prime \prime} \in \mathcal{T}$ with $\operatorname{Ext}_{A}^{1}\left(M^{\prime \prime}, M\right) \neq 0$. Again due to Auslander-Smalø, there exists a relative Auslander-Reiten sequence in $\mathcal{T}$ starting with $M$. By (2), the image under $F$ is an Auslander-Reiten sequence starting in $F(M)=N_{B}$, and all its terms are in $\mathcal{Y}$. Thus $\tau^{-1} N_{B} \in \mathcal{Y}$.

(4) Let $N_{B}$ be indecomposable and in $\mathcal{X}$. Then $\tau^{-1} N_{B}$ is in $\mathfrak{X}$. Also, either $\tau N_{B}$ is in X, or $N_{B}=F^{\prime} P(a), \tau N_{B}=F I(a)$ with $P(a) \notin$ add $T_{A}$.

Proof. Assume $0 \neq \tau^{-1} N_{B} \in \mathcal{Y}$. Then $N_{B}=\tau \tau^{-1} N_{B} \in \mathcal{Y}$, according to (3). Similarly, let $0 \neq \tau N_{B} \in \mathcal{O}$. Then either $\tau N_{B}=F I(a), N_{B}=\tau^{-1} \tau N_{B}=F^{\prime} P(a)$ and $P(a)$ $\notin$ add $T_{A}$, or else $N_{B}=\tau^{-1} \tau N_{B} \in \mathcal{Y}$, again according to (3).

Note that both $\mathcal{X}$ and $\mathcal{Y}$ are closed under extensions. This finishes the proof of the theorem.

(6.6) Assume now in addition that $A$ is connected. Note that in this case all modules of the form $F I(a)$ belong to the same connected component $C$ of the Auslander-Reiten quiver of $B$, due to the form of the connecting sequences. Let us describe this component in more detail:

LEMMA. Let $C$ be the component of the Auslander-Reiten quiver of $B$ containing all modules FI $(a)$. Then any module of $e$ can be written, in a unique way, as $\tau^{z} F I(a)$, with $z \in \mathbf{Z}$, and $I(a)_{A}$ indecomposable injective.

Proof. Let $e^{\prime}$ be the indecomposable modules of the form $\tau^{z} F I(a)$. First, we show that $e=e^{\prime}$. Let $N_{B} \rightarrow \tau^{m} F I(a)$ be an irreducible map, with $N_{B}$ indecomposable. By induction on $m \in \mathbf{N}$, we show that $N_{B} \in \mathcal{C}^{\prime}$. Note that in case $N_{B}$ is injective, then either $N_{B} \approx F I(b)$ for some $b$, or else $N_{B} \in \mathcal{X}$, according to 2.4 . Since $\tau^{m} F I(a) \in \mathcal{Y}$ for all $m \in \mathbf{N}$, we see that $N_{B}$ can only be injective in case $N_{B} \approx F I(b)$ for some $b$. Thus we may assume that $N_{B}$ is not injective, and therefore there exists an irreducible map $\tau^{m} F I(a) \rightarrow \tau^{-1} N_{B}$. In case $m=0$, it follows from 6.1 that either $\tau^{-1} N_{B} \approx F I(b)$ for some $b$, or $\tau^{-1} N_{B} \approx F^{\prime} P(c) \approx \tau^{-1} F I(c)$ for some $c$, thus $\tau^{-1} N_{B} \in e^{\prime}$ (and therefore $N_{B} \in e^{\prime}$ ). In case $m>0$, there exists an irreducible map $\tau^{-1} N_{B} \rightarrow$ $\tau^{m-1} F I(a)$, thus by induction $\tau^{-1} N_{B} \in e^{\prime}$.

Similarly, let $\tau^{-m} F I(a) \rightarrow N_{B}$ be an irreducible map with $N_{B}$ indecomposable, and $m \in \mathbf{N}$. Again, by induction on $m$, we show $N_{B} \in \mathcal{C}$. This is clear for $m=0$. For $m \geqslant 1, \tau^{-m} F I(a) \approx \tau^{-m+1} F^{\prime} P(a)$ belongs to $\mathfrak{X}$, thus $N_{B}$ cannot be projective (all projectives belong to $\mathscr{Y})$. Therefore, there exists an irreducible map $\tau^{-m+1} F I(a) \rightarrow$ $\tau N_{B}$, and by induction $\tau N_{B} \in e^{\prime}$.

Finally, in case there is an irreducible map $N_{B} \rightarrow \tau^{-m} F I(a)$, with $m \geqslant 1$, then there also exists an irreducible map $\tau^{-m+1} F I(a) \rightarrow N_{B}$. And, in case there is an irreducible map $\tau^{m} F I(a) \rightarrow N_{B}, m \geqslant 1$, then there is an irreducible map $N_{B} \rightarrow \tau^{m-1} F I(a)$. In 
both situations we are reduced to cases discussed above, and see that $N_{B} \in \mathcal{C}^{\prime}$. Thus $e=e^{\prime}$.

Uniqueness. It clearly is sufficient to show that $\tau^{-m} F I(a) \approx F I(b)$ with $m \in \mathbf{N}$ is only possible for $m=0$ and $I(a)=I(b)$. Now if $m=0$, then $I(a) \approx I(b)$, since $F$ is an equivalence between $\mathcal{T}$ and $\mathcal{Y}$. On the other hand, if $m>0$, then $F I(b) \in \mathcal{Y}$, and $\tau^{-m} F I(a) \in \mathcal{X}$, thus there is no nonzero map $\tau^{-m} F I(a) \rightarrow F I(b)$.

\section{Complete slices.}

(7.1) Let $B$ be a finite dimensional algebra, $C$ a component of its Auslander-Reiten quiver. A complete slice $\mathcal{Q}$ in $C$ consists of a set of indecomposable modules in $\mathcal{C}$ with the following properties:

(i) Given any indecomposable module $X$ in $\mathcal{C}$, then $\mathscr{U}$ contains precisely one module from the orbit $\left\{\tau^{z} X \mid z \in \mathbf{Z}\right\}$ of $X$ under $\tau, \tau^{-1}$.

(ii) If $X_{0} \rightarrow X_{1} \rightarrow \cdots \rightarrow X_{r}$ is a chain of nonzero maps and indecomposable modules, and $X_{0}, X_{r}$ belong to $\mathscr{Q}$, then all $X_{i}$ belong to $\mathcal{Q}$.

(iii) There is no oriented cycle of irreducible maps $U_{0} \rightarrow U_{1} \rightarrow \cdots \rightarrow U_{r} \rightarrow U_{0}$ with all $U_{i}$ in $\mathscr{Q}$, and $r \geqslant 1$.

If $\mathcal{Q}$ is a finite complete slice, then we will call the direct sum $U=\bigoplus_{U(a) \in \mathcal{Q}} U(a)$ the corresponding slice module.

Proposition. Let $A$ be hereditary and connected, and $T_{A}$ a tilting module with $B=\operatorname{End}\left(T_{A}\right)$. Then $\left\{F(I(a)) \mid a \in \Gamma_{0}\right\}$ is a complete slice in its component.

Proof. (i) has been shown in 6.6.

(ii) Let $F(I(a)) \stackrel{f}{\rightarrow} X$ be a nonzero map, with $X$ indecomposable. Assume $X$ is not of the form $F I(b)$. We claim that then $X \in \mathcal{X}$. We prove this by induction on $|F I(a)|$. Since $f$ is not an isomorphism, we can factor $f$ through the minimal left almost split map starting with $f$,

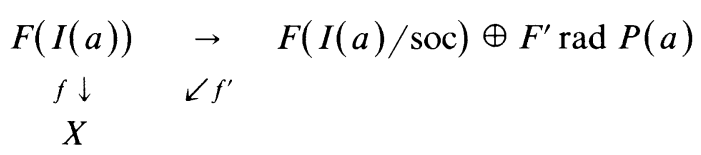

and, since $f \neq 0$, there is either a direct summand $F I(b)$ of $F\left(I(a) /\right.$ soc) or $F^{\prime} P(b)$ of $F^{\prime} \operatorname{rad} P(a)$ such that the restriction of $f^{\prime}$ to this summand is nonzero. In the first case, we note that $|I(b)|<|I(a)|$, thus by induction $x \in \mathcal{X}$. In the second case, it follows from $F^{\prime} P(b) \in \mathcal{X}$ that $X$ cannot belong to $\mathscr{Y}$, thus $X \in \mathcal{X}$ also in this case.

As a consequence, if $X_{0} \rightarrow X_{1} \rightarrow \cdots \rightarrow X_{r}$ is a chain of indecomposable modules and nonzero maps, and $X_{0}=F I(a)$, then either all $X_{i}$ are of the form $F I(*)$, or else there is some $X_{i} \in \mathcal{X}$, and then clearly all the following modules belongs to $\mathfrak{X}$.

(iii) There are no cycles of nonzero and noninvertible maps between the modules $I(*)$, since the quiver of $A$ cannot have oriented cycles, thus there cannot be a cycle of nonzero and noninvertible maps between the modules $F I(*)$.

(7.2) TheOREM. Let $B$ be a finite dimensional algebra. Assume some component $\mathcal{C}$ of the Auslander-Reiten quiver of $B$ contains all indecomposable projective modules and also some finite complete slice $\mathcal{Q}$, say with slice module $U$. Then $U_{B}$ is a tilting module, and $\operatorname{End}\left(U_{B}\right)$ is hereditary. (Thus, $B$ is a tilted algebra.) 
Let us formulate explicitly an important consequence: If we assume that $B$ has precisely $n$ simple modules, say with corresponding primitive idempotents $e_{1}, \ldots, e_{n}$, then $A=\operatorname{End}\left(U_{B}\right)$ also has precisely $n$ simple modules, and the slice module $U_{B}$ gives rise to $n$ indecomposable $A$-modules ${ }_{A} U e_{1}, \ldots,{ }_{A} U e_{n}$.

The proof will be done in several steps. We will need several auxiliary results which will be obtained first.

(7.3) Lemma. Let $A$ be hereditary, $T_{A}$ a tilting module and $B=\operatorname{End}\left(T_{A}\right)$. Let $P(a)$ be simple projective and not in add $T_{A}$. Let $I_{A}^{\prime}$ be the direct sum of all indecomposable injective A-modules different from $I(a)$. Then the B-module $F\left(I^{\prime}\right) \oplus F^{\prime} P(a)$ is a tilting module.

Proof. Let $\sigma: \Re_{A} \rightarrow \Re_{\sigma A}$ be the reflection [7] with respect to $P(a)$. Note that this also is a tilting functor, with tilting module $P^{\prime} \oplus \tau^{-1} P(a)$, where $P^{\prime}$ is the direct sum of all indecomposable projective $A$-modules different from $P(a)$, with $\sigma A=$ $\operatorname{End}\left(P^{\prime} \oplus \tau^{-1} P(a)\right)$, and $\sigma=\operatorname{Hom}_{A}\left(P^{\prime} \oplus \tau^{-1} P(a),-\right)$, compare with [16]. There is a simple injective module $Z$ in $\Re_{\sigma A}$ such that $\sigma$ induces an equivalence between the full subcategory $\Re_{A}^{\prime}$ of $\Re_{A}$ of all modules without direct summand of the form $P$, and the full subcategory $\Re_{\sigma A}^{\prime}$ of $\Re_{\sigma A}$ of all modules without direct summand of the form $Z$.

Since $P(a)$ is not a direct summand of $T_{A}$, we know that $T_{A}$ is in $\Re_{A}^{\prime}$, thus $B=\operatorname{End}\left(T_{A}\right) \approx \operatorname{End}\left(\sigma T_{\sigma A}\right)$. Also, the indecomposable modules in $\widetilde{T}\left(T_{A}\right)$ correspond bijectively under $\sigma$ to the indecomposable modules in $\sigma\left(\sigma T_{\sigma A}\right)$ different from $Z$. Note that $\sigma T_{\sigma A}$ again is a tilting module. Let

$$
\tilde{F}=\operatorname{Hom}_{\sigma A}\left({ }_{B} \sigma T_{\sigma A},-\right): \Re_{\sigma A} \rightarrow \Re_{B} .
$$

Then clearly the functor $\tilde{F} \sigma$ is equivalent to the functor $F=\operatorname{Hom}_{A}\left({ }_{B} T_{A},-\right)$.

Now let $I(a)_{A}$ be injective, with $\operatorname{soc} I(a)=P(a) / \operatorname{rad} P(a)$. Then $\sigma I(a)$ is not injective, in fact $\tau^{-1} \sigma I(a)=Z$. [This is well known, but also follows from the connecting lemma applied to the tilting module ${ }_{\sigma A}\left(P^{\prime} \oplus \tau^{-1} P(a)\right)_{A}$ : it asserts that $\tau^{-1} \sigma I(a)=\sigma^{\prime} P(a)=Z$.]

We claim that $F^{\prime} P(a) \approx \tilde{F} Z$. By the connecting lemma, we have $F^{\prime} P(a)=$ $\tau^{-1} F I(a)$, thus $=\tau^{-1} \tilde{F} \sigma I(a)$. Since $\sigma I(a)$ is not injective, there is an AuslanderReiten sequence in $\Re_{B}$ starting with $\tilde{F} \sigma I(a)$, due to 2.4 , and it is not a connecting sequence. Thus, according to 6.5 , all its terms are images under $\tilde{F}$, thus it clearly is the image of the Auslander-Reiten sequence in $\Re_{\sigma A}$, starting with $\sigma I(a)$, under the functor $\tilde{F}$. Consequently,

$$
\tau^{-1} \tilde{F} \sigma I(a)=\tilde{F} \tau^{-1} \sigma I(a)
$$

Altogether we see that

$$
F^{\prime} P(a)=\tau^{-1} F I(a)=\tau^{-1} \tilde{F} \sigma I(a)=\tilde{F} \tau^{-1} \sigma I(a)=\tilde{F} Z .
$$

Now, $I_{A}^{\prime}$ denotes the direct sum of all indecomposable injective modules different from $I(a)$. Then $\sigma I_{\sigma A}^{\prime}$ is the direct sum of all indecomposable injective modules different from $Z$. Thus $\sigma I_{\sigma A}^{\prime} \oplus Z_{\sigma A}$ is the minimal injective cogenerator of $\Re_{\sigma A}$. By a remark in 4.5 , its image under $\tilde{F}$ is a tilting module, this is

$$
\tilde{F}\left(\sigma I_{\sigma A}^{\prime} \oplus Z_{\sigma A}\right)=\tilde{F} \sigma I^{\prime} \oplus \tilde{F} Z=F I^{\prime} \oplus F^{\prime} P(a) .
$$


(7.4) We will prove Theorem 7.2 by induction. For this, we need the more general concept of a (not necessarily complete) slice.

First recall that if $X$ is an indecomposable module, and $\mathcal{V}$ a set of indecomposable modules, then $X$ will be called a predecessor of $\mathcal{V}$, provided there exists a chain of irreducible maps and indecomposable modules

$$
X=X_{0} \rightarrow X_{1} \rightarrow \cdots \rightarrow X_{r-1} \rightarrow X_{r}
$$

with $X_{r} \in \mathcal{T}$. If in addition, $X$ does not belong to $\mathscr{V}$, then $X$ is called a proper predecessor of $\mathcal{T}$.

Now, again, let $B$ be a finite dimensional algebra, and $C$ a component of its Auslander-Reiten quiver. A slice $\mathcal{Q}$ in $C$ is a set of indecomposable modules in $C$ satisfying the conditions (ii), (iii) of 7.1, and, in addition the condition

(i') If $X$ is a predecessor of $\mathscr{U}$, then $\mathcal{U}$ contains precisely one module from the orbit $\left\{\tau^{z} X \mid z \in \mathbf{Z}\right\}$.

Clearly, this is a more general concept than that of a complete slice, since a predecessor of $\mathcal{Q}$ always belongs to the component of $\mathcal{C}$ again. Note that in contrast to a complete slice, the modules belonging to a slice do not need to be connected by chains of irreducible maps (going either way). Again, if $\mathscr{Q}$ is a slice, then $U=$ $\bigoplus_{U(a) \in \mathcal{Q}} U(a)$ is called the corresponding slice module.

LEMMA. If $थ$ is a slice, then $X$ is a predecessor of $\mathcal{Q}$ if and only if $X$ is of the form $\tau^{t} U(a)$ for some $t \in \mathbf{N}$ and some $U(a) \in \mathcal{Q}$.

Note that if an indecomposable module $X$ is of the form $\tau^{t} U(a)$, then $t$ is uniquely determined by condition (i'), and we will write $t(X)=t$.

Proof of the Lemma. If $X=\tau^{t} U(a)$ for some $t \in \mathbf{N}$, and $U(a) \in \mathcal{U}$, then clearly $X$ is predecessor of $\mathcal{Q}$, since there is a chain of $2 t$ irreducible maps

$$
\tau^{t} U(a) \rightarrow \cdots \rightarrow U(a)
$$

Conversely, let $X$ be a predecessor of $\mathcal{Q}$, say of $U(b)$. By condition (i') there is $z \in \mathbf{Z}$ with $\tau^{z} X \in \mathcal{Q}$, say $\tau^{z} X=U(a)$, thus $X=\tau^{-z} U(a)$. Now suppose $z \geqslant 0$. Then we have a chain of irreducible maps

$$
U(a) \rightarrow \cdots \rightarrow \tau^{-z} U(a)=X \rightarrow \cdots \rightarrow U(b) .
$$

Condition (ii) shows that $X \in \mathcal{Q}$, thus $z=0$.

(7.5) If $\mathcal{Q}$ is a slice, then $U(a) \in \mathcal{U}$ is called a sink for $\mathscr{U}$ provided for any irreducible map $U(a) \rightarrow X$ with $X$ indecompsable, we have $X \notin \mathcal{Q}$.

LEMma. Let $\mathscr{Q}$ be a slice, $U(a) \in \mathcal{Q}$ a sink for $\mathscr{U}$. If $U(a)$ is projective, let $\tilde{\mathcal{U}}$ be obtained from थ be deleting $U(a)$. If $U(a)$ is not projective, let थี be obtained from $\mathscr{Q}$ by replacing $U(a)$ by $\tau U(a)$. Then $\tilde{Q}$ is again a slice.

Proof. (1) If $f: U(a) \rightarrow X$ is nonzero and noninvertible, then $X \notin \mathcal{Q}$.

Proof. We can factor $f$ through the minimal left almost split map $\left(g_{i}\right)_{i}: U(a) \rightarrow$ $\oplus Z_{i}$, say $f=\sum f_{i} g_{i}$. For some $i$, we have $f_{i} g_{i} \neq 0$. If $X \in \mathcal{Q}$, then

$$
U(a) \stackrel{g_{i}}{\rightarrow} Z_{i} \stackrel{f_{i}}{\rightarrow} X
$$

implies that $Z_{i} \in \mathcal{Q}$, according to the slice condition (ii). However $U(a)$ is a sink. 
(2) If $Y \rightarrow U(a)$ is irreducible with $Y$ indecomposable, then $Y \in \mathcal{Q}$.

Proof. $Y$ is a predecessor of $\tilde{Q}$, thus $Y=\tau^{t} U(b)$ for some $t \in \mathbf{N}$ and some $b$. There is a chain of irreducible maps

$$
U(b) \rightarrow \cdots \rightarrow \tau^{-t} U(b)=Y \rightarrow U(a),
$$

thus $Y \in \mathcal{Q}$, according to (ii).

(3) If $U(a)$ is projective, then $\tilde{\mathcal{U}}$ is a slice.

Proof. For condition ( $\left.i^{\prime}\right)$, let $X$ be an indecomposable module which is a predecessor of $\tilde{\mathcal{U}}$, thus also of $\mathcal{Q}$. Since $\mathcal{U}$ is a slice, $\tau^{-t} X \in \mathcal{U}$ for some $t \in \mathbf{N}$. But $\tau^{-t} X=U(a)$ is impossible: namely, since $U(a)$ is projective, we would have $t=0$, however $U(a)$ is not a predecessor of $\tilde{Q}$.

For condition (ii), let $X_{0} \rightarrow X_{1} \rightarrow \cdots \rightarrow X_{r}$ be a chain of irreducible maps and indecomposable modules, with $X_{0}, X_{r} \in \tilde{\mathcal{Q}}$. Since $\tilde{\mathcal{U}} \subseteq \mathcal{Q}$, all $X_{i}$ belong to $\mathcal{Q}$. Assume $X_{i}=U(a), X_{i+1} \in \tilde{\mathcal{U}}$ for some $i$. Since there is a nonzero map $X_{i} \rightarrow X_{i+1}$, we get a contradiction to (1).

Condition (iii) is trivially satisfied.

(4) If $U(a)$ is not projective, then again $\tilde{Q}$ is a slice.

Proof. For condition ( $\left.i^{\prime}\right)$ we only note that a predecessor $X$ of $\tilde{Q}$ is also one for $\mathscr{Q}$, thus $\left\{\tau^{z} X \mid z \in \mathbf{Z}\right\}$ contains precisely one module from $\mathscr{Q}$, thus also just one from थี.

For condition (ii), let

$$
X_{0} \stackrel{f_{1}}{\rightarrow} X_{1} \rightarrow \cdots \stackrel{f_{r}}{\rightarrow} X_{r}
$$

be a chain of nonzero maps and indecomposable modules. Consider first the case that $X_{0}, X_{r}$ both belong to $\mathcal{Q} \cap \tilde{\mathcal{U}}$. Then all $X_{i}$ belong to $\mathcal{Q}$ according to (ii), and no $X_{i}=U(a)$ according to (1), thus all $X_{i}$ belong to $\tilde{\mathcal{U}}$. Consider now the general case. Of course, we may suppose that no $\operatorname{map} f_{i}$ is invertible.

If $X_{0}=\tau U(a)$, let

$$
0 \rightarrow \tau U(a) \stackrel{\left(g_{j}\right)_{j}}{\rightarrow} \oplus U_{j} \rightarrow U(a) \rightarrow 0
$$

be the corresponding Auslander-Reiten sequence, the middle term being of the form $\oplus U_{j}$, with $U_{j} \in \mathcal{Q}$, according to (2). Of course, all $U_{j} \in \mathcal{Q} \cap \tilde{\mathcal{U}}$. We factor now $f_{1}=\Sigma h_{j} g_{j}$, and obtain at least one nonzero $h_{j}: U_{j} \rightarrow X_{1}$. Similarly, if $X_{r}=\tau U(a)$, then for at least one $t$, the map $g_{t} f_{r}$ is nonzero. Consider now the chain

$$
U_{j} \stackrel{h_{j}}{\rightarrow} X_{1} \stackrel{f_{2}}{\rightarrow} X_{2} \rightarrow \cdots \stackrel{f_{r-1}}{\rightarrow} X_{r-1} \stackrel{g_{t} f_{r}}{\rightarrow} U_{t}
$$

here $U_{j}, U_{t} \in \mathcal{Q} \cap \tilde{\mathcal{Q}}$, thus by the previous consideration, all $X_{i} \in \tilde{\mathcal{U}}$.

For condition (iii), assume there exists an oriented cycle $U_{0} \rightarrow U_{1} \rightarrow \cdots \rightarrow U_{r} \rightarrow U_{0}$ of irreducible maps and all $U_{i} \in \tilde{\mathcal{U}}$. If one of the modules, say $U_{0}$, belongs to $\mathcal{Q} \cap \tilde{\mathcal{U}}$, then all belong to $\mathcal{U}$, according to (ii), but this is impossible due to (iii). Thus all $U_{i}=\tau U(a)$. But this again is impossible, since there are no irreducible endomorphisms.

This finishes the proof. 
(7.6) LEMMA. If $थ$ is a finite slice with slice module $U$, then $\operatorname{Ext}^{1}(U, U)=0$.

Proof. Let $U(a), U(b) \in \mathcal{Q}$. The group $D \operatorname{Ext}^{1}(U(a), U(b))$ is a factor group of $\operatorname{Hom}(U(b), \tau U(a))$. Thus, let $\varphi: U(b) \rightarrow \tau U(a)$ be a nonzero map. Then we obtain a chain of nonzero maps and indecomposable modules

$$
U(b) \rightarrow \tau U(a) \rightarrow * \rightarrow U(a)
$$

using the Auslander-Reiten sequence ending in $U(a)$. Thus $\tau U(a) \in \mathcal{U}$, according to condition (ii), but this contradicts condition (i').

(7.7) LEMMA. If थ is a finite slice with slice module $U_{B}$, and if $U_{B}$ is also a tilting module, then $\operatorname{End}\left(U_{B}\right)$ is hereditary.

Proof. Let $A=\operatorname{End}\left(U_{B}\right)$. Now the indecomposable projective $A$-modules have the form $P(a)=\operatorname{Hom}\left({ }_{A} U_{B}, U(a)_{B}\right)$, with $U(a) \in \mathcal{Q}$. Also, if $Y_{A}$ is an indecomposable $A$-submodule of $P(a)_{A}$, then $Y_{A}=\operatorname{Hom}\left({ }_{A} U_{B}, N_{B}\right)$ for some indecomposable $N_{B}$, and the inclusion $Y_{A} \hookrightarrow P(a)_{A}$ is of the form $\operatorname{Hom}\left({ }_{A} U_{B}, f\right)$, where $f: N_{B} \rightarrow U(a)_{B}$ is a nonzero map. From $Y_{A} \neq 0$, we obtain some nonzero map $g: U(b)_{B} \rightarrow N_{B}$, thus we have the chain

$$
U(b)_{B} \stackrel{g}{\rightarrow} N_{B} \stackrel{f}{\rightarrow} U(a)_{B},
$$

and therefore $N_{B} \in \mathcal{Q}$, according to condition (ii). This shows that $Y_{A}$ again is projective.

(7.8) Lemma. Let $\mathcal{Q}$ be a slice with a finite number of predecesors. Let $U_{B}$ be the corresponding slice module. Then p.d. $U_{B} \leqslant 1$, and if $Q_{B}$ is a projective module which is a predecessor of $Q$, then there exists an exact sequence

$$
0 \rightarrow Q_{B} \rightarrow U_{B}^{\prime} \rightarrow U_{B}^{\prime \prime} \rightarrow 0
$$

with $U^{\prime}, U^{\prime \prime} \in \operatorname{add} U_{B}$.

Proof. For both assertions we use induction on the number $p$ of predecessors of $\mathcal{Q}$. If $p=1$, then $\mathscr{Q}$ consists of a single module which is simple projective, thus we have nothing to show.

Now assume the result is known for slices with $p-1$ predecessors, and let $\mathcal{Q}$ be a slice with $p$ predecessors. According to condition (iii), there exists a sink $U(a)$ for $\mathcal{Q}$. Let $\tilde{\mathcal{U}}$ be obtained from $\mathcal{Q}$ by deleting $U(a)$, and, in case $U(a)$ is not projective, adding $\tau U(a)$. According to 7.5, we know that $\tilde{Q}$ again is a slice and it is clear that $\tilde{Q}$ has precisely $p-1$ predecessors. Let $\tilde{U}$ be the slice module for $\tilde{\imath}$.

Consider first the case that $U(a)$ is projective, thus $\tilde{\mathcal{U}} \subset \mathcal{U}$. By induction, p.d. $\tilde{U}_{B} \leqslant 1$, and $U_{B}=\tilde{U}_{B} \oplus U(a)$, thus p.d. $U_{B} \leqslant 1$. Also if $Q_{B}$ is projective and a predecessor of $\mathscr{Q}$, then either $Q=U(a)$, or else $Q_{B}$ is even a predecessor of $\tilde{Q}$. In the first case, we use the exact sequence

$$
0 \rightarrow U(a) \stackrel{\text { id }}{\rightarrow} U(a) \rightarrow 0 \rightarrow 0
$$

in the second case, we have, by induction, an exact sequence

$$
0 \rightarrow Q_{B} \rightarrow U_{B}^{\prime} \rightarrow U_{B}^{\prime \prime} \rightarrow 0
$$

with $U^{\prime}, U^{\prime \prime} \in \operatorname{add} \tilde{U}_{B} \subseteq$ add $U_{B}$. 
Thus, it remains to consider the case of $U(a)$ not being projective. Let Ann $\tilde{U}_{B}$ be the annihilator of $\tilde{U}_{B}$, and $\bar{B}=B /$ Ann $\tilde{U}_{B}$. Also, let $Q(1), \ldots, Q(m)$ be the projective $B$-modules which are predecessors of $U_{B}$ and $Q=\bigoplus_{i=1}^{m} Q(i)$. By induction, any $Q(i), 1 \leqslant i \leqslant m$, has an exact sequence

$$
0 \rightarrow Q(i) \rightarrow \tilde{U}^{\prime} \rightarrow \tilde{U}^{\prime \prime} \rightarrow 0
$$

with $\tilde{U}^{\prime}, \tilde{U}^{\prime \prime} \in$ add $\tilde{U}_{B}$; thus we see that $Q_{B}$ is cogenerated by $\tilde{U}_{B}$, and therefore is a $B$-module. Note that if $X$ is a predecessor of $\tilde{Q}$, and $Q(i)$ is indecomposable projective satisfying $\operatorname{Hom}(Q(i), X) \neq 0$, then $Q(i)$ has to be a predecessor of $\tilde{U}$, due to the fact that $X$ has only finitely many predecessors. As a consequence, all predecessors of $\tilde{\mathcal{Q}}$ are generated by $Q_{B}$, and therefore are, in fact, $\bar{B}$-modules, and also $Q(1), \ldots, Q(m)$ are all the indecompasable projective $\bar{B}$-modules. Finally, consider the Auslander-Reiten sequence

$$
0 \rightarrow \tau U(a) \rightarrow U^{\prime} \rightarrow U(a) \rightarrow 0
$$

in $\Re_{B}$, and note that $U^{\prime} \in$ add $\tilde{U}$ [we use that $U(a)$ is a sink for $\tilde{Q}$, see 7.5 (2)]. Since $U^{\prime}$ is a $\bar{B}$-module, the same it true for $U(a)$, and thus the sequence is also an Auslander-Reiten sequence in $\Re_{\bar{B}}$. In particular, denoting by $\tau_{B}^{-}$and $\tau_{\bar{B}}^{-1}$ the Auslander-Reiten translation inside the category $\Re_{B}^{-}$, we have $\tau_{\bar{B}}^{=1} \tau U(a)=U(a)$.

We claim that $\tilde{U}_{B}^{-}$is a tilting module. By induction, p.d. $\tilde{U}_{B} \leqslant 1$; thus also p.d. $\tilde{U}_{B}^{-} \leqslant 1$. Also, we have the exact sequence $(*)$, and these are sequences of $\bar{B}$-modules. Finally, $\operatorname{Ext}_{B}^{1}\left(\tilde{U}_{B}, \tilde{U}_{B}\right)=0$ according to 7.6 , thus also $\operatorname{Ext}_{B}^{1}-\left(\tilde{U}_{B}^{-}, \tilde{U}_{B}^{-}\right)=0$. Also note that according to 7.7 , the $\operatorname{ring} \bar{A}=\operatorname{End}\left(\tilde{U}_{B}^{-}\right)$is hereditary. Let $P(a)_{A}^{-}=$ Hom $_{B}\left({ }_{A}-\tilde{U}_{B}^{-}, \tau U(a)_{B}^{-}\right)$, then $P(a)_{A}^{-}$is simple projective, since $\tau U(a)$ is a source for $\tilde{Q}$. The corresponding indecomposable injective module $I(a)_{A}^{-}$with $\operatorname{soc} I(a)=$ $P(a) / \operatorname{rad} P(a)$ is given by $I(a)_{A}^{-}=D \operatorname{Hom}_{B}\left(\tau U(a)_{B}^{-},{ }_{A}^{-} \tilde{U}_{B}^{-}\right)$.We want to apply 7.3. We consider the tilting module ${ }_{B} T_{A}^{-}=D\left({ }_{A} \tilde{U}_{B}^{-}\right)$, and the functors $F, F^{\prime}: \Re_{\bar{A}} \rightarrow \Re_{\bar{B}}$ given by $F\left(M_{\bar{A}}^{-}\right)=\operatorname{Hom}_{\bar{A}}\left(\bar{B}_{\bar{A}} T_{\bar{A}}^{-}, M_{\bar{A}}^{-}\right)$, and $F^{\prime}\left(M_{\bar{A}}^{-}\right)=\operatorname{Ext}_{A}^{1}\left({ }_{B} T_{\bar{A}}^{-}, M_{\bar{A}}^{-}\right)$. Then

$$
\begin{aligned}
F\left(I(a)_{\bar{A}}\right) & =\operatorname{Hom}_{\bar{A}}\left({ }_{B} T_{\bar{A}}^{-}, I(a)_{\bar{A}}\right) \\
& =\operatorname{Hom}_{\bar{A}}^{-}\left(D\left({ }_{A} \tilde{U}_{\bar{B}}^{-}\right), D\right) \operatorname{Hom}_{\bar{B}}-\left(\tau U(a)_{\bar{B}},{ }_{A} \tilde{U}_{\bar{B}}^{-}\right) \\
& \approx \operatorname{Hom}_{\bar{A}}^{-}\left(\operatorname{Hom}_{\bar{B}}^{-}\left(\tau U(a)_{\bar{B}},{ }_{A} \tilde{U}_{\bar{B}}^{-}\right),{ }_{A} \tilde{U}_{\bar{B}}^{-}\right) \approx \tau U(a)_{\bar{B}},
\end{aligned}
$$

the last isomorphism being due to the fact that $\tau U(a)_{B}^{-}$is a direct summand of $\tilde{U}_{B}^{-}$, and $\operatorname{End}\left(\tilde{U}_{B}^{-}\right)=\bar{A}$ with respect to its canonical operation: namely consider the canonical map

$$
X_{B}^{-} \rightarrow \operatorname{Hom}_{A}^{-}\left(\operatorname{Hom}_{B}^{-}\left(X_{B}^{-},{ }_{A}^{-} \tilde{U}_{B}^{-}\right), \tilde{U}_{\bar{B}}^{-}\right), \quad x \mapsto(\varphi \mapsto \varphi(x)) .
$$

It is an isomorphism for $X_{\bar{B}}^{-}=\tilde{U}_{\bar{B}}^{-}$, and therefore for all $X_{B}^{-}$in add $\tilde{U}_{\bar{B}}^{-}$.

The connecting lemma shows that

$$
F^{\prime}\left(P(a)_{\bar{A}}\right)=\tau_{\bar{B}}^{-1} F\left(I(a)_{\bar{A}}\right)=\tau_{\bar{B}}^{-1} \tau U(a)=U(a)_{\bar{B}},
$$

in particular, $P(a)_{\bar{A}}^{-}$does not belong to add $T_{\bar{A}}^{-}$. Let $I_{\bar{A}}^{\prime}$ be the direct sum of the indecomposable injective $\bar{A}$-modules different from $I(a)$. Then

$$
F\left(I^{\prime} \oplus I(a)\right) \approx \tilde{U}_{B}^{-}
$$


thus, if $U_{B}=\bigoplus_{b} U(b)$, then $F\left(I^{\prime}\right) \approx \oplus_{b \neq a} U(b)$, and therefore

$$
F\left(I^{\prime}\right) \oplus F^{\prime}(P(a)) \approx\left(\bigoplus_{b \neq a} U(b)\right) \oplus U(a)=U_{B}^{-} .
$$

According to 7.3 , we know that this $\bar{B}$-module is a tilting module. Thus every $\bar{B}$-module $Q(i), 1 \leqslant i \leqslant m$, has an exact sequence

$$
0 \rightarrow Q(i) \rightarrow U^{\prime} \rightarrow U^{\prime \prime} \rightarrow 0
$$

with $U^{\prime}, U^{\prime \prime} \in$ add $U_{B}^{-}$. Of course, these sequences are exact sequences of $B$-modules, this finishes one part of the induction. Also, p.d. $U_{B}^{-} \leqslant 1$, so there is an exact sequence

$$
0 \rightarrow Q_{B}^{\prime \prime} \rightarrow Q_{\bar{B}}^{\prime} \rightarrow U_{B}^{-} \rightarrow 0
$$

with $Q^{\prime}, Q^{\prime \prime} \in$ add $Q_{\bar{B}}^{-}$. However $Q$ is projective even as a $B$-module, thus also $Q_{B}^{\prime}, Q_{B}^{\prime \prime}$ are projective, and therefore this sequence is a projective resolution of $U_{B}$, as a $B$-module; thus p.d. $U_{B} \leqslant 1$.

(7.9) End of the proof of Theorem 7.2: It remains to be seen that under the conditions of Theorem 7.2 , the slice $Q$ has only finitely many predecessors. Namely, then it follows from 7.8 that p.d. $U_{B} \leqslant 1$, and that any projective module $Q_{B}$ which is a predecessor of $\mathcal{U}$ has an exact sequence

$$
0 \rightarrow Q_{B} \rightarrow U_{B}^{\prime} \rightarrow U_{B}^{\prime \prime} \rightarrow 0
$$

with $U^{\prime}, U^{\prime \prime} \in$ add $U_{B}$. However, any indecomposable projective $B$-module $Q_{B}$ belongs to $\mathcal{C}$, thus is of the form $\tau^{z} U(a)$ for some $z \in \mathbf{Z}$ and some $U(a) \in \mathcal{Q}$, according to condition (i), and actually $z \geqslant 0$, since $Q_{B}$ is projective. This shows that any such module $Q_{B}$ is a predecessor of $\mathcal{Q}$. Finally, $\operatorname{Ext}_{B}^{1}(U, U)=0$ according to 7.6. This shows that $U_{B}$ is a tilting module. By 7.7, its endomorphism ring is hereditary. Thus, we only have to prove the following lemma.

LEMMA. If $\mathscr{Q}$ is a finite complete slice in the component $\mathcal{C}$, and $\mathcal{C}$ contains all indecomposable projective modules, then \& has only finite many predecessors.

Proof. Recall that any predecessor $X$ of $\mathscr{U}$ is of the form $X=\tau^{t(X)} U(a)$ for some $U(a) \in \mathcal{Q}$ and some number $t(X) \in \mathbf{N}$. Both $U(a)$ and $t(X)$ are uniquely determined by $X$.

(1) Let $X, Y$ be indecomposable modules, and $X \rightarrow Y$ an irreducible map. If $X$ is a proper predecessor of $\mathcal{Q}$, then $Y$ is a predecessor of $\mathcal{Q}$, and $t(X) \geqslant t(Y)$.

Proof. We have $X=\tau^{t(X)} U(a)$ for some $t(X) \in \mathbf{N}, U(a) \in \mathscr{Q}$. Since $X \notin \mathscr{Q}$, even $t(X)>0$, thus $X$ is not injective and there are irreducible maps

$$
Y \rightarrow \tau^{t(X)-1} U(a) \rightarrow \cdots \rightarrow U(a),
$$

thus also $Y$ is predecessor of $\mathscr{Q}$. Therefore $Y=\tau^{t(Y)} U(b)$ for some $t(Y) \in \mathbf{N}, U(b)$ $\in \mathcal{Q}$. Assume we would have $t(X)<t(Y)$. Then there are irreducible maps

$$
U(a) \rightarrow \tau^{t(Y)-t(X)} U(b) \rightarrow \cdots \rightarrow U(b),
$$

thus $\tau^{t(Y)-t(X)} U(b)$ is in $थ$, according to condition (ii), but this contradicts condition (i).

(2) If $X, Y$ are predecessors of $\mathcal{Q}$, and $\operatorname{Hom}(X, Y) \neq 0$, then $t(X) \geqslant t(Y)$. 
Proof. Assume $t(X)<t(Y)=t$. If $X$ is not in $\mathscr{Q}$, there exists an irreducible map $X=X_{0} \stackrel{g_{1}}{\rightarrow} X_{1}$ and a map $f_{1}: X_{1} \rightarrow Y$ with $f_{1} g_{1} \neq 0$. Namely, let $\left(g_{i}^{\prime}\right)_{i}: X \rightarrow \oplus X_{i}^{\prime}$ be minimal left almost split, and $f: X \rightarrow Y$ nonzero. Since $f$ cannot be an isomorphism according to $t(X)<t(Y)$, there are maps $f_{i}^{\prime}: X_{i}^{\prime} \rightarrow Y$ with $f=\sum f_{i}^{\prime} g_{i}^{\prime}$. Thus $f_{i}^{\prime} g_{i}^{\prime} \neq 0$ for some $i$, and we let $g_{1}=g_{i}^{\prime}, f_{1}=f_{i}^{\prime}$. By induction, we construct a chain of irreducible maps

$$
X=X_{0} \stackrel{g_{1}}{\rightarrow} X_{1} \stackrel{g_{2}}{\rightarrow} X_{2} \rightarrow \cdots \stackrel{g_{m}}{\rightarrow} X_{m}
$$

and a map $f_{m}: X_{m} \rightarrow Y$ such that $f_{m} g_{m} \cdots g_{1} \neq 0$, as long as no $X_{i}, 0 \leqslant i<m$, belongs to $\mathcal{Q}$. By (1), all $X_{i}, 0 \leqslant i \leqslant m$, are predecessors of $\mathcal{Q}$, and $t\left(X_{i}\right) \leqslant t(X)$. [For, assume $X_{m}, f_{m}, g_{m}$ are constructed, and $X_{m} \notin \mathcal{Q}$. Let $\left(h_{i}\right)_{i}: X_{m} \rightarrow \oplus Z_{i}$ be minimal left almost split. Since $f_{m}$ is not an isomorphism, we obtain maps $h_{i}^{\prime}: Z_{i} \rightarrow Y$ with $f_{m}=\sum h_{i}^{\prime} h_{i}$. Now $0 \neq f_{m} g_{m} \cdots g_{1}=\sum h_{i}^{\prime} h_{i} g_{m} \cdots g_{1}$, thus there is $i$ with $h_{i}^{\prime} h_{i} g_{m}$ $\cdots g_{1} \neq 0$. Let $X_{m+1}=Z_{i}, g_{m+1}=h_{i}, f_{m+1}=h_{i}^{\prime}$.] Since $Q$ is finite, and $t\left(X_{i}\right)$ is bounded by $t$, there are only finitely many possibilities for $X_{i}$, thus the Harada-Sai Lemma [10] shows that this process must stop, thus after a finite number of steps we obtain $X_{m} \in \mathcal{Q}$. Now $\operatorname{Hom}\left(X_{m}, Y\right) \neq 0$ together with the fact that $Y$ is a predecessor of $\mathcal{Q}$ implies that also $Y \in \mathcal{Q}$, according to condition (ii), thus $t(X)=0=t(Y)$, a contradiction.

(3) $थ$ has only finitely many predecessors.

Proof. Let $Q$ be indecomposable projective. Since $Q \in \mathcal{C}$, we have $\tau^{z} Q \in \mathcal{Q}$ for some $z \in \mathbf{Z}$, according to condition (i). However, the projectivity of $Q$ then implies $z \leqslant 0$, thus $Q$ is a predecessor of $\mathcal{Q}$. Let $X$ be any predecessor of $Q$. Then there exists an indecomposable projective module $Q$ with $\operatorname{Hom}(Q, X) \neq 0$, and therefore $t(X) \leqslant t(Q)$, by (2). This shows that $t(X)$, for $X$ predecessor of $\mathcal{Q}$, is bounded by $\max t(Q)$, where $Q$ runs through all indecomposable projective modules. Since $Q$ is finite, there only can be a finite number of predecessors of $\mathscr{Q}$.

This finishes the proof of the lemma, and also the proof of Theorem 7.2.

(7.11) Corollary. Let $B$ be a connected finite dimensional algebra, of finite representation type. Then $B$ is a tilted algebra if and only if the Auslander-Reiten quiver of $B$ contains a complete slice.

Proof. This follows directly from 7.1 and 7.2, since the Auslander-Reiten quiver of a connected algebra of finite representation type is connected.

REMARK. We have introduced the notion of a complete slice in order to be able to formulate Theorem 7.2. Other authors have considered similar, however, weaker notions such as "sections" [4] or "complete sections" [6]. In the example of the algebra $B$ given by the quiver<smiles>[2H][Te]1CCC[Ge]CC1</smiles>

with relations $\alpha \beta=0, \alpha^{2}=0$, the indecomposable projective modules form a complete section in the sense of [6], however, $B$ is not a tilted algebra (nor obtained from a hereditary algebra by a sequence of tilts in the sense of [6]), since tilted algebras 
with only two simple modules clearly have to be hereditary. This contradicts a conjecture of [6]. Also note that $B$ has a section in the sense of [4] consisting of three modules. Also related is a construction by Reidtmann [11] which however deals with the universal covering of the Auslander-Reiten quiver of $B$, and not with the Auslander-Reiten quiver itself. We will imitate her procedure in the next section when constructing a complete slice starting from an indecomposable sincere representation. Note however, that even for a tilted algebra of finite representation type, the Auslander-Reiten quiver does not have to coincide with its universal covering. In [8], Gabriel uses the notion of a slice for the special case of a translation quiver $\mathbf{Z} Q$, with $Q$ a Dynkin quiver, in a very similar way as we do.

8. Components without oriented cycles. Let $B$ be a finite dimensional algebra. Recall that an oriented cycle in the Auslander-Reiten quiver of $B$ is given by a sequence of indecomposable modules and irreducible maps

$$
X_{0} \rightarrow X_{1} \rightarrow X_{2} \rightarrow \cdots \rightarrow X_{m} \rightarrow X_{0}
$$

with $m \geqslant 1$.

(8.1) Proposition. Let $C$ be a component of the Auslander-Reiten quiver of $B$, and assume there exists a complete slice in $\mathcal{C}$. Then there are no oriented cycles in $\mathcal{C}$.

Proof. Let $\mathscr{Q}$ be a slice in $\mathcal{C}$, and assume there exists an oriented cycle

$$
X_{0} \rightarrow X_{1} \rightarrow X_{2} \rightarrow \cdots \rightarrow X_{m} m \rightarrow X_{0}
$$

inside $\mathcal{C}$. Now for any $i$, there is $z_{i} \in \mathbf{Z}$ such that $\tau^{z_{i}} X_{i}$ belongs to $\mathcal{Q}$. If $z_{i}>0$ for all $i$, then let $z=\min _{0 \leqslant i \leqslant m} z_{i}$, and apply $\tau^{z}$ to the cycle. Similarly, if $z_{i}<0$, let $z=\max _{0 \leqslant i \leqslant m} z_{i}$ and apply $\tau^{z}$ to the cycle. We therefore see that we can assume that there is $i$ with $z_{i} \geqslant 0$ and $j$ with $z_{j} \leqslant 0$. Using Auslander-Reiten sequences, we obtain chains of irreducible maps

$$
\tau^{z_{i}} X_{i} \rightarrow \cdots \rightarrow X_{i}, \quad X_{j} \rightarrow \cdots \rightarrow \tau^{z_{j}} X_{j},
$$

thus combining with irreducible maps from the given oriented cycle we obtain a chain of irreducible maps

$$
\tau^{z_{i}} X_{i} \rightarrow \cdots \rightarrow X_{i} \rightarrow \cdots \rightarrow X_{0} \rightarrow X_{1} \rightarrow \cdots \rightarrow X_{m} \rightarrow X_{0} \rightarrow \cdots \rightarrow X_{j} \rightarrow \cdots \rightarrow \tau^{z_{j}} X_{j},
$$

and the slice condition (ii) implies that all $X_{i}$ belong to $\mathcal{Q}$, contrary to the slice condition (iii).

(8.2) ThEOREM. Let $\mathrm{C}$ be a preprojective component of the Auslander-Reiten quiver of $B$ containing a sincere indecomposable representation $N_{B}$.

Then $C$ contains all indecomposable projective modules and also a complete slice, thus $B$ is a tilted algebra. In fact, there is a hereditary algebra $A$, a tilting module $T_{A}$, and a simple injective module $I_{A}$, such that $B=\operatorname{End}\left(T_{A}\right)$, and $N_{B}=\operatorname{Hom}_{A}\left({ }_{B} T_{A}, I_{A}\right)$.

Proof. We fix a sincere indecomposable representation $N_{B}$ in $C$. Let us call a chain $X_{0} \rightarrow X_{1} \rightarrow \cdots \rightarrow X_{m}$ of irreducible maps and indecomposable modules a path from $X_{0}$ to $X_{m}$. Such a path will be called sectional provided $X_{i-1} \approx \tau X_{i+1}$ for all $1 \leqslant i \leqslant m-1$. 
(1) Let $Q_{B}$ be indecomposable projective. Since $N_{B}$ is sincere, $\operatorname{Hom}(Q, N) \neq 0$. Thus, since $C$ is a preprojective component, there is a path from $Q$ to $N$ in $C$. In particular, $Q$ belongs to $C$.

(2) Let $Q$ be the set of all modules $U$ in $\mathcal{C}$ with the following two properties:

$(\alpha)$ There exists a path from $U_{B}$ to $N_{B}$, and

$(\beta)$ any path from $U_{B}$ to $N_{B}$ is sectional.

We claim that $\mathcal{Q}$ is a complete slice.

ProOF of CONDITION (i): First, consider an indecomposable projective module $Q_{B}$. According to (1), there is a path from $Q$ to $N$. Now choose $t$ maximal such that there is a path from $\tau^{-t} Q$ to $N$. The maximum exists, since $N$ has only finitely many predecessors. We claim that any path from $\tau^{-t} Q$ to $N$ is sectional. Otherwise, there is a path of the form

$$
\tau^{-t} Q=X_{0} \rightarrow \cdots \rightarrow X_{i} \rightarrow X_{i+1} \rightarrow \tau^{-1} X_{i} \rightarrow \cdots \rightarrow X_{m}=N .
$$

Now the modules $X_{j}, 1 \leqslant j \leqslant i$, cannot be injective. [Namely $N_{B}$ is sincere, thus if $X_{j}$ is injective, then $\operatorname{Hom}_{B}\left(N, X_{j}\right) \neq 0$, thus, since $X_{j} \in \mathcal{C}$, there exists a path from $N$ to $X_{j}$. On the other hand, by assumption, there is a proper path from $X_{j}$ to $X_{m}=N$, thus we have an oriented cycle in $C$, contradiction.] Thus, for $1 \leqslant j \leqslant i$, we obtain from the irreducible map $X_{j-1} \rightarrow X_{j}$, an irreducible map $\tau^{-1} X_{j-1} \rightarrow \tau^{-1} X_{j}$. Thus, altogether we obtain a path

$$
\tau^{-(t+1)} Q=\tau^{-1} X_{0} \rightarrow \cdots \rightarrow \tau^{-1} X_{i}
$$

which we can combine with the given path from $\tau^{-1} X_{i}$ to $X_{m}=N$ in order to have a path from $\tau^{-(t+1)} Q$ to $N$, contrary to the maximality of $t$. This shows that $\tau^{-t} Q \in \mathcal{Q}$. The same argument also shows that $\tau^{-t} Q$ is the only module in the orbit of $Q$ under $\tau^{-1}$ which belongs to $\mathscr{Q}$. Namely, for $t^{\prime}<t$, there clearly is a nonsectional path from $\tau^{-t^{\prime}} Q$ to $N$, whereas for $t^{\prime}>t$, there is no path at all from $\tau^{-t^{\prime}} Q$ to $N$. The condition (i) for a general module $Y$ in $\bigodot$ follows directly from the fact that $Y$ is in the $\tau$-orbit of a projective module.

ProOF OF CONDITION (ii). Let

$$
X_{0} \stackrel{g_{1}}{\rightarrow} X_{1} \stackrel{g_{2}}{\rightarrow} \cdots \stackrel{g_{m}}{\rightarrow} X_{m}
$$

be a chain of indecomposable modules and nonzero maps, with $X_{0}, X_{m}$ in $\mathcal{Q}$. Since $X_{m} \in \mathcal{C}$, and $\operatorname{Hom}\left(X_{i-1}, X_{i}\right) \neq 0$ for $1 \leqslant i \leqslant m$, all $X_{i}$ belong to $\mathcal{C}$, and there is a path from $X_{i-1}$ to $X_{i}$. We delete isomorphisms and replace the noninvertible maps $g_{i}$ by chains of irreducible maps, thus obtaining a path from $X_{0}$ to $X_{m}$ which contains all the given modules $X_{i}$. Thus, without loss of generality, we may assume all $g_{i}$ to be irreducible. We want to show that all $X_{i}$ belong to $\mathcal{Q}$. Since $X_{m}$ is in $\mathcal{Q}$, there exists a path from $X_{m}$ to $N$. Combining this with the given path from $X_{i}$ to $X_{m}$, we obtain a path from $X_{i}$ to $N$, thus showing $(\alpha)$. Assume there exists a nonsectional path from $X_{i}$ to $N$. Combining this with the given path from $X_{0}$ to $X_{i}$, we clearly obtain a nonsectional path from $X_{0}$ to $N$, contradicting that $X_{0} \in \mathcal{Q}$. This shows $(\beta)$.

The condition (iii) follows directly from the fact that there are no oriented cycles in all of $e$. 
This finishes the proof that $\mathcal{Q}$ is a complete slice. Let $U_{B}$ be the corresponding slice module, and $A=\operatorname{End}\left(U_{B}\right)$. According to 7.2, we know that $U_{B}$ is a tilting module, and $A$ is hereditary. Let ${ }_{B} T_{A}=D\left({ }_{A} U_{B}\right)$, then also $T_{A}$ is a tilting module, and $\operatorname{End}\left(T_{A}\right)=B$. By construction of $U_{B}$, we know that $N_{B}$ is a direct summand of $U_{B}$, thus $\operatorname{Hom}_{B}\left(N_{B},{ }_{A} U_{B}\right)$ is a projective left $A$-module which obviously is also simple. Thus $I_{A}=D \operatorname{Hom}_{B}\left(N_{B},{ }_{A} U_{B}\right)$ is a simple injective right $A$-module. Finally note that

$$
\begin{aligned}
\operatorname{Hom}_{A}\left({ }_{B} T_{A}, I_{A}\right) & =\operatorname{Hom}_{A}\left(D\left({ }_{A} U_{B}\right), D \operatorname{Hom}_{B}\left(N_{B},{ }_{A} U_{B}\right)\right) \\
& \approx \operatorname{Hom}_{A}\left(\operatorname{Hom}_{B}\left(N_{B},{ }_{A} U_{B}\right),{ }_{A} U_{B}\right) \approx N_{B},
\end{aligned}
$$

the first isomorphism being due to duality, the second follows from the fact that $N_{B}$ is a direct summand of $U_{B}$, and $A=\operatorname{End}\left(U_{B}\right)$. This finishes the proof.

REMARK. Using idempotents of $A$, we may reformulate the last assertion of the theorem as follows: Under the assumptions of the theorem, there exists a hereditary algebra $A$, a tilting module $T_{A}$, and a primitive idempotent $e$ of $A$ such that

$$
B=\operatorname{End}\left(T_{A}\right), \quad N_{B}=D\left({ }_{B} T e\right) .
$$

Namely, let $P_{A}$ be the projective module with $P_{A} / \operatorname{rad} P_{A} \approx \operatorname{soc} I_{A}\left(=I_{A}\right)$, and let $e$ be a primitive idempotent of $A$ with $P_{A} \approx e A$. Then we have

$$
\operatorname{Hom}_{A}\left({ }_{B} T_{A}, I_{A}\right) \approx D \operatorname{Hom}_{A}\left(P_{A},{ }_{B} T_{A}\right) \approx D \operatorname{Hom}_{A}\left(e A_{A},_{B} T_{A}\right) \approx D\left({ }_{B} T e\right) .
$$

(8.3) COROllary. Let $B$ be of finite representation type with an indecomposable sincere representation $N_{B}$. Then $B$ is a tilted algebra if and only if the Auslander-Reiten quiver of $B$ does not contain oriented cycles. Also, in this case, there exists a hereditary algebra $A$, a tilting module $T_{A}$, and a simple injective $A$-module $I_{A}$, such that $B=\operatorname{End}\left(T_{A}\right)$, and $N_{B}=\operatorname{Hom}\left({ }_{B} T_{A}, I_{A}\right)$.

Proof. Since $B$ has an indecomposable sincere representation, $B$ is connected, thus the Auslander-Reiten quiver of $B$ is connected due to the fact that $B$ is of finite representation type. Now, if $B$ is a tilted algebra, then 7.1 and 8.1 show that there are no oriented cycles. Conversely, we use 8.2 in order to show that $B$ is a tilted algebra in case there are no oriented cycles in its Auslander-Reiten quiver. Namely, since $B$ is of finite representation type, any indecomposable module is either of the form $\tau^{-t} Q$ with $t \in \mathbf{N}$ and $Q$ indecomposable projective, or else $\tau$-periodic. But the existence of $\tau$-periodic modules would imply the existance of oriented cycles in the Auslander-Reiten quiver. The second assertion follows again from Theorem 8.2.

(8.4) THEOREM. Let $B$ be a finite dimensional algebra of finite representation type, and assume the Auslander-Reiten quiver of $B$ has no oriented cycles. Let $N_{B}, N_{B}^{\prime}$ be indecomposable B-modules. Then $\operatorname{dim} N_{B}=\operatorname{dim} N_{B}^{\prime}$ implies $N_{B} \approx N_{B}^{\prime}$.

Proof. Without loss of generality, we may assume that both $N_{B}$ and $N_{B}^{\prime}$ are sincere. For, let $\bar{B}=B / E$ where $E$ is the twosided ideal of $B$ generated by all idempotents $e$ with $N e=0$. Then $N$ becomes a sincere $\bar{B}$-module, and, since $\operatorname{dim} N_{B}=\operatorname{dim} N_{B}^{\prime}$, we know that $E$ also annihilates $N^{\prime}$ and that also $N^{\prime}$ is sincere as a $\bar{B}$-module. Obviously, with $B$ also $\bar{B}$ is of finite representation type. Also, an oriented cycle in the Auslander quiver of $\bar{B}$ would give rise to an oriented cycle in the 
Auslander-Reiten quiver of $B$. According to 8.3, we know that $B$ is a tilted algebra and $N_{B}=\operatorname{Hom}\left({ }_{B} T_{A}, I_{A}\right)$ where $T_{A}$ is a tilting module, $B=\operatorname{End}\left(T_{A}\right)$, and $I_{A}$ is simple injective. Since $\operatorname{Hom}\left({ }_{B} T_{A}, I_{A}\right) \neq 0$ and $I_{A}$ is simple, we see that $I_{A}$ belongs to $\mathcal{T}\left(T_{A}\right)$, thus $N_{B}=\operatorname{Hom}\left({ }_{B} T_{A}, I_{A}\right)$ belongs to $\mathcal{X}$, according to the Brenner-Butler theorem. Recall that according to 3.2 , there exists an isometry $f: G_{0}(A) \rightarrow G_{0}(B)$, such that for all $A$-modules $M_{A}$

$$
f\left(\operatorname{dim} M_{A}\right)=\operatorname{dim} \operatorname{Hom}_{A}\left({ }_{B} T_{A}, M_{A}\right)-\operatorname{dim} \operatorname{Ext}_{A}\left({ }_{B} T_{A}, M_{A}\right) .
$$

We claim that also $N_{B}^{\prime}$ belongs to $\mathcal{X}$. [Otherwise, it would belong to $\mathscr{Y}$, thus $N_{B}^{\prime}=\operatorname{Ext}^{1}\left({ }_{B} T_{A}, M_{A}\right)$ for some $M_{A} \in \mathcal{F}\left(T_{A}\right)$; thus

$$
\operatorname{dim} I_{A}=f^{-1} \operatorname{dim} N_{B}=f^{-1} \operatorname{dim} N_{B}^{\prime}=\operatorname{dim} M_{A},
$$

impossible.] This shows that $N_{B}^{\prime}=\operatorname{Hom}\left({ }_{B} T_{A}, M_{A}\right)$ for some $M_{A}$ in $\mathcal{T}\left(T_{A}\right)$, and

$$
\operatorname{dim} M_{A}=f^{-1} \operatorname{dim} N_{B}^{\prime}=f^{-1} \operatorname{dim} N_{B}=\operatorname{dim} I_{A} .
$$

However, this implies $M_{A} \approx I_{A}$, and therefore $N_{B}^{\prime} \approx N_{B}$.

REMARK. This generalizes a recent result of Bautista [4]. Namely, we note that Bautista and Larrion [5] have given a sufficient condition for an algebra $B$ in order that its Auslander-Reiten quiver has no oriented cycles. In particular, if $B$ is a factor algebra of the path algebra of a quiver with underlying graph a tree, and is of finite representation type, then its Auslander-Reiten quiver has no oriented cycles.

(8.5) As another consequence of 8.2 we obtain the following useful result on indecomposable representations of algebras of finite representation type with Auslander-Reiten quiver without oriented cycles.

TheOREM. Let $C$ be a preprojective component of the Auslander-Reiten quiver of $B$ and $N$ a module in $C$. Then:

(a) For $e_{1}, e_{2}$ primitive idempotents of $B$, and $b \in B$ the multiplication map $\left(\cdot e_{1} b e_{2}\right): N e_{1} \rightarrow N e_{2}, x e_{1} \mapsto x e_{1} b e_{2}$ is either a monomorphism, an epimorphism or zero.

(b) If $N_{B}$ is sincere, then $N_{B}$ is actually faithful.

Proof. Let $\bar{B}=B / \operatorname{Ann} N$. By 1.3 we know that the component of the Auslander-Reiten quiver of $\bar{B}$ containing $N$ again is preprojective, and in addition $N_{B}^{-}$is sincere. Thus we can assume from the beginning that $N$ is a sincere $B$-module.

According to 8.2 and using the remark at the end of 8.2 , there exists a hereditary algebra $A$, a primitive idempotent $e$ of $A$, and a tilting module ${ }_{A} T$ such that $B=\operatorname{End}\left({ }_{A} T\right)$, and $N_{B}=e T_{B}$. If $e_{i}$ is a primitive idempotent of $B$, then ${ }_{A} T e_{i}$ is an indecomposable direct summand of ${ }_{A} T$, and for $b \in B$, the multiplication map

$$
\left(\cdot e_{1} b e_{2}\right): T e_{1} \rightarrow T e_{2}
$$

is an $A$-module homomorphism. It follows from 4.1 that this map is either a monomorphism, an epimorphism, or zero. Clearly, the same is true if we multiply both $T e_{1}, T e_{2}$ from the left by $e$; this proves (a). Now assume, in addition, that $N_{B}=e T_{B}$ is sincere, thus $N e_{i}=e T e_{i}$ is nonzero for every primitive idempotent $e_{i}$. If $e_{1} b e_{2} \neq 0$, then the multiplication map $\left(\cdot e_{1} b e_{2}\right)$ on $T e_{1}$ has to be a monomorphism 
or an epimorphism, thus also the restriction

$$
\left(\cdot e_{1} b e_{2}\right): e T e_{1} \rightarrow e T e_{2}
$$

is a monomorphism or an epimorphism in particular: nonzero. As a consequence, $N_{B}$ is faithful.

(8.6) A tilted algebra $B$ has global dimension $\leqslant 2$, thus on $G_{0}(B)$ there is defined the bilinear form $\langle$,$\rangle with$

$$
\langle\operatorname{dim} X, \operatorname{dim} Y\rangle=\operatorname{dim}_{k} \operatorname{Hom}(X, Y)-\operatorname{dim}_{k} \operatorname{Ext}^{1}(X, Y)+\operatorname{dim}_{k} \operatorname{Ext}^{2}(X, Y),
$$

and correspondingly a quadratic form

$$
q(\operatorname{dim} X)=\langle\operatorname{dim} X, \operatorname{dim} X\rangle .
$$

THEOREM. Let $B$ be a tilted algebra over an algebraically closed field $k$ of finite representation type, and $x$ a positive element in $G_{0}(B)$. Then $q(x) \geqslant 1$ and there exists an indecomposable representation $X$ with $\operatorname{dim} X=x$ if and only if $q(x)=1$.

Proof. If $X$ is indecomposable, then $\operatorname{End}(X)$ is a division ring, and $\operatorname{Ext}^{1}(X, X)=$ $0, \operatorname{Ext}^{2}(X, X)=0$ since there are no oriented cycles in the Auslander-Reiten quiver of $B$. Since $k$ is algebraically closed, we even have $\operatorname{End}(X)=k$, thus $q(\operatorname{dim} X)=1$. Conversely, let $x$ be a positive element in $G_{0}(B)$. Choose a representation $X$ with $\operatorname{dim} X=x$, and $\operatorname{End}(X)$ of smallest possible dimension. Assume $\operatorname{Ext}^{1}(X, X) \neq 0$. Since $\operatorname{Ext}^{1}\left(X^{\prime}, X^{\prime}\right)=0$ for all indecomposable modules $X^{\prime}$, there exists a decomposition $X=X^{\prime} \oplus X^{\prime \prime}$ with $\operatorname{Ext}^{1}\left(X^{\prime}, X^{\prime \prime}\right) \neq 0$. Let

$$
0 \rightarrow X^{\prime \prime} \rightarrow Y \rightarrow X^{\prime} \rightarrow 0
$$

be a nonsplit exact sequence. Then $\operatorname{dim} Y=\operatorname{dim} X=x$, and $\operatorname{dim} \operatorname{End}(Y)<$ $\operatorname{dim} \operatorname{End}\left(X^{\prime} \oplus X^{\prime \prime}\right)=\operatorname{dim} \operatorname{End}(X)$ (see [12]), contrary to the choice of $X$. Thus $\operatorname{Ext}^{1}(X, X)=0$. Since $\operatorname{dim} \operatorname{End}(X) \geqslant 1, \operatorname{dim} \operatorname{Ext}^{2}(X, X) \geqslant 0$, we see that $q(x)=$ $q(\operatorname{dim} X) \geqslant 1$. In case $q(x)=1$, we conclude that $\operatorname{End}(X)=k$, thus $X$ is indecomposable.

REMARK. Note that the proof above also shows that for a fixed $x \in G_{0}(B)$, with $q(x)=1$, the variety of all representations of dimension type $x$ is irreducible.

Namely there exists a unique indecomposable representation $X$ with $\operatorname{dim} X=x$, and if $X^{\prime}$ is decomposable with $\operatorname{dim} X^{\prime}=x$, then $\operatorname{Ext}^{1}\left(X^{\prime}, X^{\prime}\right) \neq 0$ shows that $X^{\prime}$ is a proper degeneration of some other module with smaller endomorphism ring, thus, by induction, a degeneration of $X$.

Of course, if $q(x)>1$, then the variety of all representations of dimension type $x$ may be reducible, as the example

$$
0 \stackrel{\alpha}{\rightarrow} 0 \stackrel{\beta}{\rightarrow} 0, \quad \alpha \beta=0, \quad x=(111)
$$

shows.

\section{REFERENCES}

1. M. Auslander and I. Reiten, Representation theory of Artin algebras. III, VI, Comm. Algebra 3 (1975), 239-294; 5 (1977), 443-518.

2. M. Auslander and S. O. Smalo, Almost split sequences in subcategories, J. Algebra 69 (1981), 426-454; Addendum, J. Algebra (to appear).

3. R. Bautista, Sections in A uslander-Reiten quivers, Lecture Notes in Math., vol. 832, Springer-Verlag, Berlin and New York, 1980, pp. 74-96. 
4. $\ldots$ Sections in A uslander-Reiten components. II (to appear).

5. R. Bautista, and F. Larrion, A uslander-Reiten quivers for certain algebras of finite representation type.

6. S. Brenner, and M. C. R. Bulter, Generalization of the Bernstein-Gelfand-Ponomarev reflection functors, Lecture Notes in Math., vol. 832, Springer-Verlag, Berlin and New York, 1980, pp. 103-169.

7. V. Dlab, and C. M. Ringel, Indecomposable representations of graphs and algebras, Mem. Amer. Math. Soc. No. 173 (1976).

8. P. Gabriel, Auslander-Reiten sequences and representation-finite algebras, Lecture Notes in Math., vol. 831, Springer-Verlag, Berlin and New York, 1980, pp. 1-71.

9. D. Happel, Zur Darstellungstheorie von Köchern endlichen und zahmen Typs. Relative Invarianten und Subgenerische Orbiten, Dissertation, Bielefeld, 1980.

10. M. Harada and Y. Sai, On categories of indecomposable modules. I, Osaka J. Math. 7 (1970), $323-344$.

11. Chr. Riedtmann, Algebren, Darstellungen, Überlagerungen und zurück, Comment. Math. Helv. 55 (1980), 199-224.

12. C. M. Ringel, The rational invariants of the tame quiver, Invent. Math. 58 (1980), 217-239.

13. __ Tame algebras, Lecture Notes in Math., vol. 831, Springer-Verlag, Berlin and New York, 1980, pp. 137-287.

14. Report on the Brauer-Thrall conjecures: Rojter's theorem and the theorem of Nazarova-Rojter, Lecture Notes in Math., vol. 831, Springer-Verlag, Berlin and New York, 1980, pp. 104-136.

15. M. Auslander, Applications of morphisms determined by models. Representation theory of algebras, Lecture Notes in Pure and Appl. Math., vol. 37, Dekker, New York, 1978.

16. M. Auslander, M. I. Platzeck and I. Reiten, Coxeter functors without diagrams, Trans. Amer. Math. Soc. 250 (1979), 1-46.

17. K. Bongartz, and C. M. Ringel, Representation-finite tree algebras, Lecture Notes in Math., vol. 903, Springer-Verlag, Berlin and New York, 1981, pp. 39-54.

18. D. Happel and C. M. Ringel, Construction of tilted algebras, Lecture Notes in Math., vol. 903, Springer-Verlag, Berlin and New York, 1981, pp. 125-144.

FAKUltät Fưr MAThematik, Universität BielefEld, D-4800 Bielefeld, Federal Republic of GERMANY 